ESAIM: PS 16 (2012) 222-276

DOI: $10.1051 / \mathrm{ps} / 2011106$
ESAIM: Probability and Statistics

www.esaim-ps.org

\title{
MANIFOLD INDEXED FRACTIONAL FIELDS *
}

\author{
JACQUES ISTAS ${ }^{1}$
}

\begin{abstract}
Local) self-similarity is a seminal concept, especially for Euclidean random fields. We study in this paper the extension of these notions to manifold indexed fields. We give conditions on the (local) self-similarity index that ensure the existence of fractional fields. Moreover, we explain how to identify the self-similar index. We describe a way of simulating Gaussian fractional fields.
\end{abstract}

Mathematics Subject Classification. 60G07, 60G15, 60G18.

Received July 9, 2009. Revised April 14, 2010.

\section{INTRODUCTION}

This paper originated in a lecture given in June 2009 in Mons (France) during the conference "Random differential equations and Gaussian fields". The purpose of such a paper is both to present a synthesis of classical results and recent results, even very recent. In addition, some open questions are mentioned. The central theme of the paper is the manifold indexed fractional fields. In the Euclidean case, there is an enormous literature, the most famous field being the fractional Brownian field of index $0<H \leq 1$. But other examples exist, in particular in the stable framework. The first question to ask on the fields is of course, after their definition, their existence. We will see that the existence of fractional fields is related to the geometry of the manifold. That is why this papers starts with non-probabilistic reminders. Also involved in particular the theorem of Schönberg, the Bernstein functions and the fractional index. A major open question in this section concerns the actual calculation of this fractional index. We then talk about the central concept of self-similarity, at least in the Euclidean case, and then define what may happen to that concept in the manifold case. In particular, we show that the fractional Brownian field exists for a $H$ between 0 and half of the fractional index, and no more between 0 and 1 . In the $\alpha$-stable case, this condition becomes $0<H \leq 1 / \alpha$. The difference with the Euclidean case is obvious. We then turn to the spectral or moving-average representations, also treated in detail in [32]. In the manifold case, we can represent the fields in the Gaussian case, and yet it lacks sometimes explicit forms. We then turn to the local self-similarity property, in short lass. The lass property is naturally written in the manifold case, via the tangent bundle. We then obtain expected results, in terms of existence. Let us now turn to the statistical estimation of parameters. This question has been much studied in the Euclidean case and we present the classical results. The issue has been poorly studied in the manifold case even if it appears at first glance that the same ideas can be reused. Finally there is the question of numerical simulation, at least in the Gaussian case. Simulating a Gaussian field seems simple: it suffices to simulate a Gaussian vector. But

Keywords and phrases. Self-similarity, stochastic fields, manifold.

* This work was supported by Research Project ANR-GDSA.

1 Laboratoire Jean Kuntzmann, Université de Grenoble et CNRS, 38041 Grenoble Cedex 9, France. Jacques.Istas@imag.fr 
even in the two-dimensional case, the vector size makes illusory direct simulation. There is not currently fast and accurate simulation algorithm for Gaussian fields. We present here a method which is fast but rough, first within the Euclidean case, then for manifolds.

\section{A REVIEW OF DISTANCE'S PROPERTIES}

\subsection{Metric spaces, Riemannian manifolds}

As usual, a metric space is a pair $(E, d)$ where $E$ is a set and $d$ is a metric on $E$, that is, a function $d: E \times E \rightarrow \mathbb{R}$ such that

- $d(M, N) \geq 0$;

- $d(M, N)=0$ if and only if $M=N$;

- $d(M, N)=d(N, M)$

- $d(M, P) \leq d(M, N)+d(N, P)$.

In this paper, we denote by $(\mathcal{M}, g)$ a $\mathcal{C}^{\infty}$-complete Riemannian manifold of dimension $n$. These strong assumptions of completude and $C^{\infty}$ are given by convenience and may be weakened in several places. The geodesic distance $d(M, N)$ is defined as the length of the shortest curve between $M$ and $N$. When the Riemannian manifold $(\mathcal{M}, g)$ is view as a metric space, it will be denoted by $(\mathcal{M}, d)$.

The most used Riemannian manifolds or metric spaces of this paper are the following.

- Euclidean spaces $\left(\mathbb{R}^{n},\|\|\right)$.

For $M=\left(x_{1}, \ldots, x_{n}\right) \in \mathbb{R}^{n}, O=(0, \ldots, 0),\|O M\|$ is the usual Euclidean norm

$$
\|O M\|^{2}=\sum_{1}^{n} x_{i}^{2}
$$

- Unit spheres $\left(\mathbb{S}^{n}, d\right)$.

The unit sphere of dimension $n$ is defined by

$$
\mathbb{S}^{n}=\left\{\left(x_{1}, \ldots, x_{n+1}\right) \in \mathbb{R}^{n+1}, \sum_{1}^{n+1} x_{i}^{2}=1\right\} .
$$

For $M=\left(x_{1}, \ldots, x_{n+1}\right), N=\left(y_{1}, \ldots, y_{n+1}\right) \in \mathbb{S}^{n}$, the geodesic distance $d(M, N)$ is defined by

$$
\cos d(M, N)=\sum_{1}^{n+1} x_{i} y_{i}
$$

- Hyperbolic spaces $\left(\mathbb{H}^{n}, d\right)$.

The hyperbolic space of dimension $n$ is defined by

$$
\mathbb{H}^{n}=\left\{\left(x_{1}, \ldots, x_{n+1}\right) \in \mathbb{R}^{n+1}, x_{n+1}>0, x_{n+1}^{2}-\sum_{1}^{n} x_{i}^{2}=1\right\} .
$$

For $M=\left(x_{1}, \ldots, x_{n+1}\right), N=\left(y_{1}, \ldots, y_{n+1}\right) \in \mathbb{H}^{n}$, the geodesic distance $d(M, N)$ is defined by

$$
\cosh d(M, N)=x_{n+1} y_{n+1}-\sum_{1}^{n} x_{i} y_{i}
$$

- Real trees $(\mathbb{T}, d)$.

A metric space $(\mathbb{T}, d)$ is a real tree (e.g. [39]) if the following two properties hold for every $M, N \in \mathbb{T}$. 
- There is a unique isometric map $f_{M, N}$ from $[0, d(M, N)]$ into $\mathbb{T}$ such that $f_{M, N}(0)=M$ and $f_{M, N}(d(M, N))=N$.

- If $\phi$ is a continuous one to one map from $[0,1]$ into $\mathbb{T}$, such that $\phi(0)=M$ and $\phi(1)=N$, we have

$$
\phi([0,1])=f_{M, N}([0, d(M, N)]) .
$$

\subsection{Functions of positive and negative type}

Let us now recall the definitions of functions of positive or negative type. Let $X$ be a set.

- A symmetric function $(M, N) \mapsto \phi(M, N), X \times X \rightarrow \mathbb{R}^{+}$is of positive type if, $\forall n \geq 2, \forall M_{1}, \ldots, M_{n} \in$ $X, \forall \lambda_{1}, \ldots, \lambda_{n} \in \mathbb{R}$

$$
\sum_{i, j=1}^{n} \lambda_{i} \lambda_{j} \phi\left(M_{i}, M_{j}\right) \geq 0 .
$$

- A symmetric function $(M, N) \mapsto \psi(M, N), X \times X \rightarrow \mathbb{R}^{+}$is of negative type ${ }^{2}$ if

$-\forall M \in X, \psi(M, M)=0$

- $\forall n \geq 2, \forall M_{1}, \ldots, M_{n} \in X, \forall \lambda_{1}, \ldots, \lambda_{n} \in \mathbb{R}$ such that $\sum_{i=1}^{n} \lambda_{i}=0$

$$
\sum_{i, j=1}^{n} \lambda_{i} \lambda_{j} \psi\left(M_{i}, M_{j}\right) \leq 0 .
$$

\subsection{Bochner's theorem}

Let $(x, y) \mapsto \phi(x, y), \mathbb{R}^{n} \times \mathbb{R}^{n} \rightarrow \mathbb{R}^{+}$be a continuous function of positive type such that $\phi(x, y)$ depends only on the difference $x-y$. The Bochner's theorem (e.g. $[7,74])$ characterizes these functions via their Fourier transform.

Theorem 2.1. Bochner's theorem.

Among the continuous real valued functions, the functions $(x, y) \mapsto \phi(x, y)$ of positive type on $\mathbb{R}^{n}$, depending on the difference $x-y$, are those functions which are the Fourier transforms of finite symmetric measures.

\subsection{Schönberg's theorem}

Let $\psi$ and $\phi$ two symmetric functions from $X \times X$ onto $\mathbb{R}^{+}$related by

$$
\phi(M, N)=\psi(O, M)+\psi(O, N)-\psi(M, N),
$$

where $O$ is a given point of $X$. In the probabilistic framework, $\phi$ will be the covariance function of a random field, and $\psi$ the variance of its increments.

Theorem 2.2. Schönberg's theorem [7,77].

(1) The following items are equivalent

- Function $\psi$ is of negative type.

- $\forall t \geq 0$, function $\exp (-t \psi)$ is of positive type.

(2) The following items are equivalent.

- $\phi$ is of positive type.

- $\psi$ is of negative type.

\footnotetext{
${ }^{2}$ Some authors say "functions of conditionally negative type".
} 


\subsection{Bernstein's functions}

Definition 2.3. Bernstein's function.

A function $x \mapsto F(x), \mathbb{R}^{+} \rightarrow \mathbb{R}^{+}$is a Bernstein function if there exist $a, b \geq 0$ and a positive measure $\mu$ satisfying

$$
\int_{0}^{+\infty} \frac{\lambda}{1+\lambda} \mu(\mathrm{d} \lambda)<\infty
$$

such that

$$
F(x)=a+b x+\int_{0}^{+\infty}\left(1-\mathrm{e}^{-\lambda x}\right) \mu(\mathrm{d} \lambda)
$$

\section{Proposition 2.4.}

The following functions are Bernstein's function

$$
\begin{aligned}
& F(x)=x^{\alpha}, \quad 0<\alpha \leq 1, \\
& F(x)=\log (1+x) .
\end{aligned}
$$

Proof of Proposition 2.4.

(1) Proposition 2.4 is straightforward for $\alpha=1$. For $0<\alpha<1$, the change of variable $y=\lambda x$ in the integral $\int_{0}^{+\infty} \frac{1-\mathrm{e}^{-\lambda x}}{\lambda^{1+\alpha}} \mathrm{d} \lambda$ leads to

$$
x^{\alpha}=C_{\alpha} \int_{0}^{+\infty} \frac{1-\mathrm{e}^{-\lambda x}}{\lambda^{1+\alpha}} \mathrm{d} \lambda,
$$

where

$$
C_{\alpha}^{-1}=\int_{0}^{+\infty} \frac{1-\mathrm{e}^{-y}}{y^{1+\alpha}} \mathrm{d} y
$$

(2) Let

$$
\mu(\mathrm{d} u)=\frac{\mathrm{e}^{-u}}{u} \mathrm{~d} u
$$

Let $\varepsilon>0$ and $x \geq 0$

$$
\int_{\varepsilon}^{+\infty}\left(1-\mathrm{e}^{-u x}\right) \frac{\mathrm{e}^{-u}}{u} \mathrm{~d} u=\int_{\varepsilon}^{+\infty} \frac{\mathrm{e}^{-u}}{u} \mathrm{~d} u-\int_{\varepsilon}^{+\infty} \frac{\mathrm{e}^{-u(x+1)}}{u} \mathrm{~d} u .
$$

Perform the change of variable $v=u(x+1)$

$$
\int_{\varepsilon}^{+\infty}\left(1-\mathrm{e}^{-u x}\right) \frac{\mathrm{e}^{-u}}{u} \mathrm{~d} u=\int_{\varepsilon}^{\varepsilon(x+1)} \frac{\mathrm{e}^{-v}}{v} \mathrm{~d} v .
$$

Perform the change of variable $w=v / \varepsilon$

$$
\int_{\varepsilon}^{+\infty}\left(1-\mathrm{e}^{-u x}\right) \frac{\mathrm{e}^{-u}}{u} \mathrm{~d} u=\int_{1}^{x+1} \frac{\mathrm{e}^{-\varepsilon w}}{w} \mathrm{~d} w .
$$


Using the dominated convergence Theorem as $\varepsilon \rightarrow 0^{+}$leads to

$$
\int_{0}^{+\infty}\left(1-\mathrm{e}^{-u x}\right) \mu(\mathrm{d} u)=\log (1+x)
$$

Proposition 2.5. Let $F$ be a Bernstein function with $a=0$. If $\psi$ is of negative type, so is $F(\psi)$.

Proof of Proposition 2.5. Let $\lambda_{1}, \ldots, \lambda_{n}$ with $\sum_{1}^{n} \lambda_{i}=0$. Then

$$
\sum_{i, j=1}^{n} \lambda_{i} \lambda_{j} F\left(\psi\left(M_{i}, M_{j}\right)\right)=b \sum_{i, j=1}^{n} \lambda_{i} \lambda_{j} \psi\left(M_{i}, M_{j}\right)-\int_{0}^{+\infty} \sum_{i, j=1}^{n} \lambda_{i} \lambda_{j} \mathrm{e}^{-\lambda \psi\left(M_{i}, M_{j}\right)} \mu(\mathrm{d} \lambda)
$$

$\sum_{i, j=1}^{n} \lambda_{i} \lambda_{j} \psi\left(M_{i}, M_{j}\right)$ is negative by assumption. $\sum_{i, j=1}^{n} \lambda_{i} \lambda_{j} \mathrm{e}^{-\lambda \psi\left(M_{i}, M_{j}\right)}$ is positive by Schönberg's theorem. Therefore, $\sum_{i, j=1}^{n} \lambda_{i} \lambda_{j} F\left(\psi\left(M_{i}, M_{j}\right)\right)$ is negative and Proposition 2.5 is proved.

\subsection{Fractional index $\boldsymbol{\beta}_{E}$}

We can now associate a fractional index $\beta_{E}$ to every metric space $(E, d)$. This fractional index is of prime importance through this paper. It is defined as

$$
\beta_{E}=\sup \left\{\beta>0 \text { such that } d^{\beta} \text { is of negative type }\right\},
$$

with the convention $\beta_{E}=0$ if $d^{\beta}$ is never of negative type.

\section{Proposition 2.6.}

- $d^{\beta}$ is of negative type for $0<\beta \leq \beta_{E}$.

- $d^{\beta}$ is never of negative type for $\beta>\beta_{E}$.

Proof of Proposition 2.6. Let $\gamma>0$ such that $d^{\gamma}$ is of negative type. Then, by Propositions 2.4 and 2.5, $d^{\alpha \gamma}$, with $0<\alpha \leq 1$, is of negative type. It follows that $d^{\beta}$ is of negative type for $0<\beta<\beta_{E}$.

Let $\left(\beta_{p}\right)_{p \geq 0}$ be an increasing sequence converging to $\beta_{E}$ (when $\beta_{E}<\infty$ ). For all $M_{1}, \ldots, M_{n} \in E$ and $\lambda_{1}, \ldots, \lambda_{n} \in \mathbb{R}$ such that $\sum_{i=1}^{n} \lambda_{i}=0$

$$
\sum_{i, j=1}^{n} \lambda_{i} \lambda_{j} d^{\beta_{p}}\left(M_{i}, M_{j}\right) \leq 0 .
$$

Let now perform $\beta_{p} \rightarrow \beta_{E}$ in $(2.2)$

$$
\sum_{i, j=1}^{n} \lambda_{i} \lambda_{j} d^{\beta_{E}}\left(M_{i}, M_{j}\right) \leq 0 .
$$

It follows that $d^{\beta_{E}}$ is of negative type and Proposition 2.6 is proved. 


\subsection{Measure definite kernel distances}

We will use in the following distances that are measure definite kernel distances. Let us therefore recall the definition of a measure definite kernel (cf. [73]). Examples will be given later.

Definition 2.7. Measure definite kernel.

A function $(M, N) \mapsto \psi(M, N)$, from $E \times E$ onto $\mathbb{R}^{+}$, is a measure definite kernel if there exists a measure space $(\mathbf{H}, \sigma(\mathbf{H}), \mu)$, that is a set $\mathbf{H}$, a $\sigma$-algebra $\sigma(\mathbf{H})$ of sets in $\mathbf{H}$, and a non-negative measure $\mu$ on $\sigma(\mathbf{H})$, and a map $M \mapsto H_{M}$ from $E$ onto $\sigma(\mathbf{H})$ such that

$$
\psi(M, N)=\mu\left(H_{M} \Delta H_{N}\right),
$$

where $\Delta$ denotes the symmetric difference of sets.

At this stage, the injectivity of the map $M \mapsto H_{M}$ is not required, although it is in practice since $\psi$ will be a distance.

Proposition 2.8. Let $\psi$ be a measure definite kernel distance. Then $\psi$ is of negative type.

Proof of Proposition 2.8. We first check that

$$
\begin{aligned}
\psi(M, N) & =\int\left|1_{H_{M}}-1_{H_{N}}\right| \mathrm{d} \mu \\
& =\int\left(1_{H_{M}}-1_{H_{N}}\right)^{2} \mathrm{~d} \mu .
\end{aligned}
$$

Take $\lambda_{1}, \ldots, \lambda_{n}$ with $\sum_{1}^{n} \lambda_{i}=0$. Then

$$
\sum_{i, j=1}^{n} \lambda_{i} \lambda_{j} \psi\left(M_{i}, M_{j}\right)=-2 \int\left(\sum_{1}^{n} \lambda_{i} 1_{H_{M_{i}}}\right)^{2} \mathrm{~d} \mu \leq 0 .
$$

\subsection{Examples}

For a given metric space, the calculation of the fractional constant $\beta_{E}$ defined by (2.1) seems to be a very difficult task. We give here most of the known results.

\subsubsection{Finiteness of $\beta_{E}$}

Proposition 2.9. Assume that $E$ contains three points $M_{1}, M_{2}, M_{3}$ such that

$$
\begin{aligned}
& d\left(M_{1}, M_{2}\right)>d\left(M_{1}, M_{3}\right), \\
& d\left(M_{1}, M_{2}\right)>d\left(M_{2}, M_{3}\right) .
\end{aligned}
$$

In other words, there exists at least one non-isosceles triangle in E. Then $\beta_{E}<\infty$.

Proof of Proposition 2.9. Take $\lambda_{1}=-1 / 2, \lambda_{2}=-1 / 2, \lambda_{3}=1$. One has

$$
\sum_{i, j=1}^{3} \lambda_{i} \lambda_{j} d^{\beta}\left(M_{i}, M_{j}\right) \sim 1 / 2 d^{\beta}\left(M_{1}, M_{2}\right)>0 \text { as } \beta \rightarrow+\infty .
$$




\subsubsection{A basic example: the metric square}

Let us give a basic example, that proves that $\beta_{E}$ can take a large range of values.

Proposition 2.10. Let $\left(M_{1}, M_{2}, M_{3}, M_{4}\right)$ be a unit metric square, i.e.

$$
\begin{aligned}
d\left(M_{1}, M_{2}\right) & =d\left(M_{2}, M_{3}\right) \\
& =d\left(M_{3}, M_{4}\right) \\
& =d\left(M_{4}, M_{1}\right) \\
& =1, \\
d\left(M_{1}, M_{3}\right) & =d\left(M_{2}, M_{4}\right) \\
& =d,
\end{aligned}
$$

with $0<d \leq 2$.

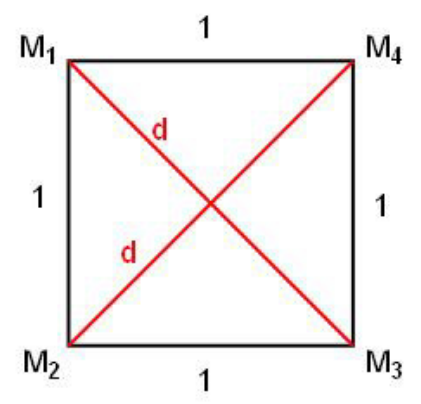

Then

- $0<d \leq 1: \beta_{E}=+\infty$;

- $1<d \leq 2: \beta_{E}=1 / \log _{2} d$.

Proof of Proposition 2.10. Take $\lambda_{1}, \ldots, \lambda_{4}$ with $\sum_{1}^{4} \lambda_{i}=0$. Let

$$
F=1 / 2 \sum_{i, j=1}^{4} \lambda_{i} \lambda_{j} d^{\beta}\left(M_{i}, M_{j}\right)
$$

Then

$$
F=-\left(\lambda_{1}+\lambda_{3}\right)^{2}+d^{\beta}\left(\lambda_{1} \lambda_{3}+\lambda_{2} \lambda_{4}\right)
$$

We can assume $\lambda_{4}=1$ without loss of generality

$$
\begin{gathered}
F=-\left(\lambda_{1}+\lambda_{3}\right)^{2}+d^{\beta}\left(\lambda_{1} \lambda_{3}-\lambda_{1}-\lambda_{3}-1\right), \\
\frac{\partial F}{\partial \lambda_{1}}=-2\left(\lambda_{1}+\lambda_{3}\right)+d^{\beta}\left(\lambda_{3}-1\right), \\
\frac{\partial F}{\partial \lambda_{3}}=-2\left(\lambda_{1}+\lambda_{3}\right)+d^{\beta}\left(\lambda_{1}-1\right) .
\end{gathered}
$$


$\frac{\partial F}{\partial \lambda_{1}}$ and $\frac{\partial F}{\partial \lambda_{3}}$ vanish for $\lambda_{1}=\lambda_{3}=d^{\beta} /\left(d^{\beta}-4\right)$. The eigenvalues of the matrix $\left(\frac{\partial^{2} F}{\partial \lambda_{i} \lambda_{j}}\right)_{i, j=1,3}$ are equal to $-d^{\beta}$ and $d^{\beta}-4$. If $d^{\beta} \leq 4$, the maximum of $F$ is given by

$$
F_{\max }=\frac{2 d^{\beta}}{d^{\beta}-4}\left(-d^{2 \beta}+6 d^{\beta}-8\right) .
$$

The polynomial $X \mapsto-X^{2}+6 X-8$ is positive for $X \in[2,4]$ and negative elsewhere.

When $0<d \leq 1, F_{\max }$ is always negative and $\beta_{E}=+\infty$.

When $1<d \leq 2, F_{\max }$ is negative for $d^{\beta} \leq 2$ and positive for $2<d^{\beta}<4$ : $\beta_{E}=1 / \log _{2} d$.

\subsubsection{Examples with $\beta_{E}=0$}

For the record, let us note the following examples with $\beta_{E}=0$.

- Spaces $\left(\mathbb{R}^{n},\|\cdot\|_{\ell^{q}}\right)$ where $\|x\|_{\ell^{q}}^{q}=\sum_{i=1}^{n}\left|x_{i}\right|^{q}$ with $n \geq 3, q>2,[60,61]$.

- Quaternionic hyperbolic space endowed with the geodesic distance $[43,44]$.

\subsubsection{Euclidean spaces}

Theorem 2.11. Let $\left(\mathbb{R}^{n},\|\|\right)$ be the usual Euclidean space. Then $\beta_{\mathbb{R}^{n}}=2$ and \|\|$^{\beta}$ is measure definite kernel distance for $0<\beta \leq 1$.

Proof of Theorem 2.11.

(1) Take $\lambda_{1}, \ldots, \lambda_{n}$ with $\sum_{1}^{n} \lambda_{i}=0$. Then

$$
\sum_{i, j=1}^{n} \lambda_{i} \lambda_{j}\left\|M_{i} M_{j}\right\|^{2}=-2\left\|\sum_{1}^{n} \lambda_{i} O M_{i}\right\|^{2} \leq 0 .
$$

Applying Propositions 2.4 and 2.5, this proves $\beta_{\mathbb{R}^{n}} \geq 2$. Take $\lambda_{ \pm 1}=-1, \lambda_{0}=2, M_{0}=O, M_{-1}=-M_{1}$. Then

$$
\sum_{i, j=-1}^{1} \lambda_{i} \lambda_{j}\left\|M_{i} M_{j}\right\|^{\beta}=2\left\|O M_{1}\right\|^{\beta}\left(2^{\beta}-4\right) .
$$

This is clearly positive when $\beta>2$, this proves $\beta_{\mathbb{R}^{n}}=2$.

(2) Let us recall the Chentsov's construction [23] that proves that the Euclidean norm \|\| is a measure definite kernel.

For any hyperplane $h$ of $\mathbb{R}^{n}$ not containing the origin, let $r$ be the distance of $h$ to the origin of $\mathbb{R}^{n}$ and let $s \in \mathbb{S}^{n-1}$ be the unit vector orthogonal to $h$. The hyperplane $h$ is parameterized by the pair $(s, r)$. Let $\mathbf{H}$ be the set of all hyperplanes that do not contain the origin. Let $\sigma(\mathbf{H})$ be the Borel $\sigma$-field. Let $\mu(\mathrm{d} s, \mathrm{~d} r)=\mathrm{d} s \mathrm{~d} r$, where $\mathrm{d} s$ denotes the uniform measure on $\mathbb{S}^{n-1}$ and $\mathrm{d} r$ the Lebesgue measure on $\mathbb{R}$. Let $H_{M}$ be the set of all hyperplanes separating the origin and the point $M$. Then, there exists a constant $c>0$ such that

$$
\|M N\|=c \mu\left(H_{M} \Delta H_{N}\right) .
$$

(3) Let us recall the Takenaka's construction [81] that proves that the fractional power of Euclidean norm \|\|$^{\beta}$ is a measure definite kernel for $0<\beta<1$. 
A hypersphere in $\mathbb{R}^{n}$ is parameterized by a pair $(x, \lambda)$, where $x \in \mathbb{R}^{n}$ is its center and $\lambda \in \mathbb{R}^{+}$its radius. Let $\mathbf{H}$ be the set of all hyperspheres in $\mathbb{R}^{n}$. Let $\sigma(\mathbf{H})$ be the Borel $\sigma$-field. $\mu_{\beta}$ is the measure $\mu_{\beta}(\mathrm{d} x, \mathrm{~d} \lambda)=$ $\lambda^{\beta-n-1} \mathrm{~d} x \mathrm{~d} \lambda$. Let $H_{M}$ be the set of hyperspheres separating the origin and the point $M$. Then, there exists a constant $c_{\beta}>0$ such that

$$
\|M N\|^{\beta}=c_{\beta} \mu_{\beta}\left(H_{M} \Delta H_{N}\right) .
$$

\subsubsection{Smooth manifolds}

Theorem 2.12. Let $(\mathcal{M}, d)$ a $\mathcal{C}^{\infty}$-complete Riemannian manifold of dimension $n$. Then $\beta_{\mathcal{M}} \leq 2$. Moreover, if there exists a point with positive Gauss curvature, then $\beta_{\mathcal{M}}<2$.

Proof of Theorem 2.12.

(1) Take $\lambda_{ \pm 1}=-1, \lambda_{0}=2$. Fix a point $M_{0} \in \mathcal{M}$, and let $M_{0}^{\varepsilon} \equiv M_{0}$. Let $v \in \mathcal{B}(0, \delta)$ and let $M_{ \pm 1}^{\varepsilon}=\exp _{M_{0}}( \pm \varepsilon v)$.

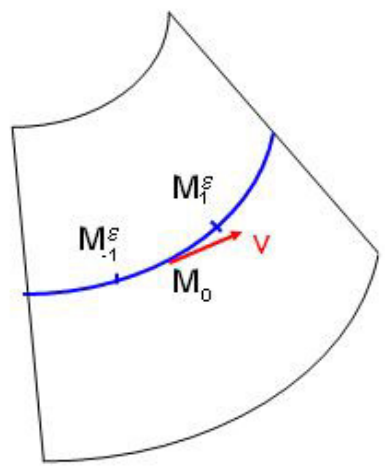

Then, by $(5.2)$

$$
\lim _{\varepsilon \rightarrow 0^{+}} \frac{\sum_{i, j=-1}^{1} \lambda_{i} \lambda_{j} d^{\beta}\left(M_{i}^{\varepsilon}, M_{j}^{\varepsilon}\right)}{\varepsilon}=2\|v\|^{\beta}\left(2^{\beta}-4\right) .
$$

This is clearly positive when $\beta>2$, this proves $\beta_{\mathcal{M}} \leq 2$.

(2) Let us first recall the following result on geodesic triangles (see figure below). We restrict ourselves to the case of manifold of dimension 2. Consider a geodesic triangle of $\mathcal{M}$ which sides have lengths $l_{1}, l_{2}, l_{3}$. Let $\alpha$ be the angle between the sides of lengths $l_{1}$ and $l_{2}$. The sides of lengths $l_{1}$ and $l_{2}$ intersect in $M$. Let $K$ be the Gauss curvature at $M$. Consider an Euclidean triangle which sides have lengths $l_{1}, l_{2}, l_{3}$. Let $\beta$ be the angle between the sides of lengths $l_{1}$ and $l_{2}$. Fix $M$ and let $\eta=\max \left(l_{1}, l_{2}, l_{3}\right)$ goes to 0 .
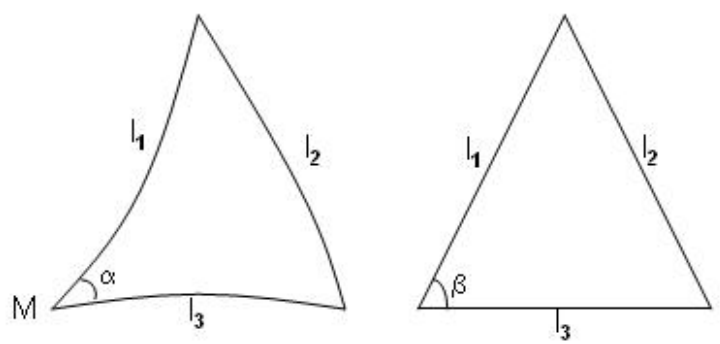
Then, the geodesic triangle formula [19] leads to

$$
\beta=\alpha+\frac{1}{6} \sin (\alpha) K l_{1} l_{2}+o\left(\eta^{2}\right)
$$

Let us now come back to our problem. Let $M_{0}$ be a given point of $\mathcal{M}$. Let us consider two geodesics $\Gamma_{1}$ and $\Gamma_{2}$ satisfying (see figure below)

(a) $M_{0} \in \Gamma_{1}, M_{0} \in \Gamma_{2}$.

(b) The tangent vector of $\Gamma_{1}$ at $M_{0}$ is orthogonal to the tangent vector of $\Gamma_{2}$ at $M_{0}$.

(c) The Gauss curvature at $M_{0}$ is equal to $K>0$.

For any $\varepsilon>0$ small enough, let us now choice four points $M_{1}^{\varepsilon}, M_{2}^{\varepsilon}, M_{3}^{\varepsilon}, M_{4}^{\varepsilon}$ such that

(a) $M_{1}^{\varepsilon}, M_{4}^{\varepsilon} \in \Gamma_{1}$,

(b) $M_{2}^{\varepsilon}, M_{3}^{\varepsilon} \in \Gamma_{2}$,

(c)

$$
\begin{aligned}
d\left(M_{0}, M_{1}^{\varepsilon}\right) & =d\left(M_{0}, M_{4}^{\varepsilon}\right) \\
& =d\left(M_{0}, M_{2}^{\varepsilon}\right) \\
& =d\left(M_{0}, M_{3}^{\varepsilon}\right) \\
& =\varepsilon,
\end{aligned}
$$

and

$$
\begin{aligned}
d\left(M_{1}^{\varepsilon}, M_{4}^{\varepsilon}\right) & =d\left(M_{2}^{\varepsilon}, M_{3}^{\varepsilon}\right) \\
& =2 \varepsilon .
\end{aligned}
$$

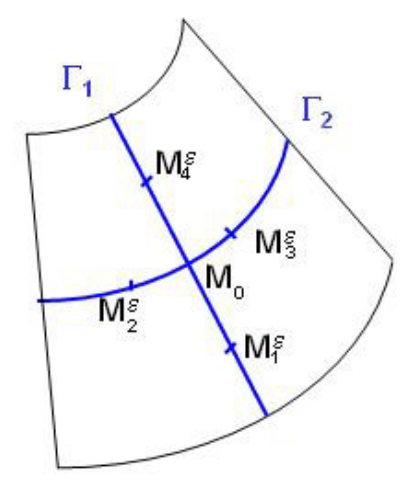

We apply the geodesic triangle formula (2.3) to each of the geodesic triangles $\left(M_{1}^{\varepsilon}, M_{0}, M_{2}^{\varepsilon}\right),\left(M_{2}^{\varepsilon}, M_{0}, M_{4}^{\varepsilon}\right)$, $\left(M_{4}^{\varepsilon}, M_{0}, M_{3}^{\varepsilon}\right)$ and $\left(M_{3}^{\varepsilon}, M_{0}, M_{1}^{\varepsilon}\right)$. For the first geodesic triangle $\left(M_{1}^{\varepsilon}, M_{0}, M_{2}^{\varepsilon}\right)$, this leads to, as $\varepsilon \rightarrow 0^{+}$

$$
\frac{d\left(M_{1}^{\varepsilon}, M_{2}^{\varepsilon}\right)}{\sqrt{2} \varepsilon}=1-\frac{K \varepsilon^{2}}{12}+o\left(\varepsilon^{2}\right)
$$

the same holds for the three other triangles. Take now $\lambda_{1}=\lambda_{4}=1$ and $\lambda_{2}=\lambda_{3}=-1$. Then, since $K>0$

$$
\lim _{\varepsilon \rightarrow 0^{+}} \frac{1}{\varepsilon^{3}} \sum_{i, j=1}^{4} \lambda_{i} \lambda_{j} d^{2}\left(M_{i}^{\varepsilon}, M_{j}^{\varepsilon}\right)>0 .
$$

$d^{2}$ is not of negative type. This proves that $\beta_{\mathcal{M}}<2$. 


\subsubsection{Spheres}

Theorem 2.13. Let $\left(\mathbb{S}^{n}, d\right)$ be the $n$-dimensional sphere. Then $\beta_{\mathbb{S}^{n}}=1$ and $d$ is a measure definite kernel distance.

Proof of Theorem 2.13. We follow [65] that proves that $d$ is measure definite kernel distance. For any point $M$ on $\mathbb{S}^{n}$, define a half-sphere by

$$
H_{M}=\left\{N \in \mathbb{S}^{n}, d(M, N) \leq \pi / 2\right\} .
$$

Let $\mathrm{d} s$ be the uniform measure on $\mathbb{S}^{n}$, let $\omega_{n}$ be the surface of the sphere, and define the measure $\mu$ by

$$
\mu(\mathrm{d} s)=\frac{\pi}{\omega_{n}} \mathrm{~d} s .
$$

The area of a sphere zone of angle $\alpha$ is equal to $\omega_{n} /(2 \pi) \alpha$. But angular distance and geodesic distance are confused on an unit sphere. Then

$$
d(M, N)=\mu\left(H_{M} \Delta H_{N}\right) .
$$

This proves that $d$ is a measure definite kernel. By Proposition 2.8, this proves $\beta_{\mathbb{S}^{n}} \geq 1$.

Let $\mathbb{S}^{1}$ be a great circle of $\mathbb{S}^{n}$. Take four equidistributed points on $\mathbb{S}^{1}$. These four points are a metric square as defined in Proposition 2.10. Since $\log _{2} 2=1$, this proves $\beta_{\mathbb{S}^{1}} \leq 1$, and therefore $\beta_{\mathbb{S}^{n}}=1$.

$n \boldsymbol{b}$ : The same is true for compact rank one symmetric spaces (in short CROSS) [46,51]. Let us recall the classification of the CROSS, also known as two points homogeneous spaces $[48,84]$ : spheres $\mathbb{S}_{d}, d \geq 1$, real projective spaces $\mathbb{P}^{d}(\mathbb{R}), d \geq 2$, complex projective spaces $\mathbb{P}^{d}(\mathbb{C}), d=2 k, k \geq 2$, quaternionic projective spaces $\mathbb{P}^{d}(\mathbb{H}), d=4 k, k \geq 2$ and Cayley projective plane $P^{16}$. [46] has proved that geodesic distance on CROSS are on negative type. The fractional index of a CROSS is therefore 1.

\subsubsection{Hyperbolic spaces}

Theorem 2.14. Let $\left(\mathbb{H}^{n}, d\right)$ be the $n$-dimensional hyperbolic space. Then $\beta_{\mathbb{H}^{n}}=1$ and $d$ is a measure definite kernel distance.

Proof of Theorem 2.14. The geodesic distance is a measure definite kernel [72,82]. The proof is more technical and we omit it. By Proposition 2.8, this proves $\beta_{\mathbb{H}^{n}} \geq 1$. Applying then [44], Proposition 7.6 one proves that $\beta_{\mathbb{H}^{n}}=1$.

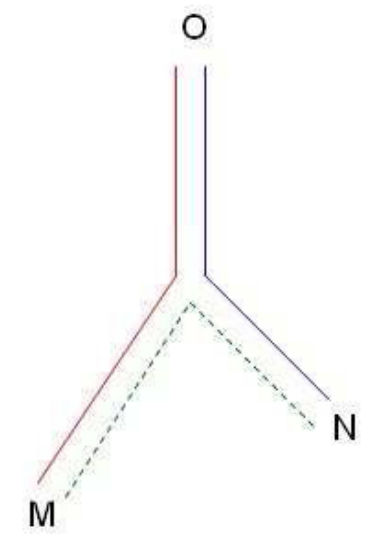




\subsubsection{Trees}

Theorem 2.15. Let $(\mathbb{T}, d)$ be a tree. $d$ is measure definite kernel distance and $\beta_{T} \geq 1$.

It has been proved by [83] that the distance on real trees is a measure definite kernel. This can be easily seen on a figure.

By Proposition 2.8, this proves $\beta_{\mathbb{T}} \geq 1$.

Computing the fractional index $\beta_{\mathbb{T}}$ for an arbitrary tree seems a difficult task. Let us nevertheless poit out two remarks.

One can build trees with $\beta_{\mathbb{T}}>1$. Let for instance $\mathbb{T}$ be the basic tree with one father and two sons. The distance between the father and a son is equal to one, the distance between the two sons is equal to two. Basic computations prove that $\beta_{\mathbb{T}}=2$.

Let us now give a family of simple trees $\left(\mathbb{T}_{p}\right)_{p \geq 1}$ such that $\lim _{p \rightarrow+\infty} \beta_{\mathbb{T}_{p}}=1$. $A_{0}$ is the root of the tree. $A_{0}$ has $p$ sons $A_{1}, \ldots, A_{p}$, with

$$
\begin{array}{ll}
d\left(A_{0}, A_{i}\right)=1 & i \neq 0, \\
d\left(A_{i}, A_{j}\right)=2 & i \neq j, i, j \neq 0 .
\end{array}
$$

Choose $\lambda_{0}=1$ and $\lambda_{i}=-1 / p$ for $i=1, \ldots, p$. Then

$$
\sum_{i, j=0}^{p} \lambda_{i} \lambda_{j} d^{\beta}\left(A_{i}, A_{j}\right)=-2+2^{\beta} \frac{p-1}{p} .
$$

$-2+2^{\beta} \frac{p-1}{p}$ is positive for $\beta \geq 1+\log _{2}\left(\frac{p}{p-1}\right)$. It follows that $\beta_{T_{p}} \leq 1+\log _{2}\left(\frac{p}{p-1}\right)$.

\section{SELF-SIMILARITY}

\subsection{Euclidean case}

\subsubsection{Generalities}

Let us start with a heuristical definition of self-similarity. A self-similar object is similar to each part of itself, i.e. the whole has the same shape as one or more of the parts. That means that a zoom in or out, from any point, leaves, up to a scale factor, the object invariant. Zoom in or out is related to scale invariance. Zoom in or out from any point is related to translation. Moreover, one should expect that no direction is favored. This property, called isotropy, is related to isometry.

Since this paper is devoted to random fields, we will restrict ourselves to graphs of functions. Let $M \mapsto$ $f(M), \mathbb{R}^{n} \rightarrow \mathbb{R}$ be a function which graph would be self-similar. One should expect the following property

(1) Invariance by isometry.

For any isometry of $\mathbb{R}^{n}$ and any $M \in \mathbb{R}^{n}$

$$
f(i(M))-f(i(O))=f(M)-f(O) .
$$

(2) Scale invariance.

There exists a measurable scale function $c(\lambda)$ such that, for any scale factor $\lambda>0$

$$
f(\lambda M)=c(\lambda) f(M) .
$$

Take two scale factors $\lambda_{1}$ and $\lambda_{2}$

$$
f\left(\lambda_{1} \lambda_{2} M\right)=c\left(\lambda_{1} \lambda_{2}\right) f(M),
$$


but

$$
\begin{aligned}
f\left(\lambda_{1} \lambda_{2} M\right) & =f\left(\lambda_{1}\left(\lambda_{2} M\right)\right) \\
& =c\left(\lambda_{1}\right) c\left(\lambda_{2}\right) f(M) .
\end{aligned}
$$

This leads to, if $f$ is non degenerate,

$$
c\left(\lambda_{1} \lambda_{2}\right)=c\left(\lambda_{1}\right) c\left(\lambda_{2}\right)
$$

$c$ is therefore a fractional power, and the scale invariance is given by

$$
f(\lambda M)=\lambda^{H} f(M) .
$$

Setting $M=O$ in (3.2) leads to $f(O)=0$. From (3.2), one deduces, for $M \neq O, f(M)=\|M\|^{H} f(M /\|M\|)$. It suffices therefore to compute $f(u)$ for any unit vector $u$. Applying (3.1) with any rotation $r$ of center $O$ leads to $f(r(u))=f(u)$. There exists therefore a constant $c$ such that

$$
f(M)=c\|M\|^{H} .
$$

We now apply (3.1) with any translation. This leads to, for any $h \in \mathbb{R}^{n}$,

$$
\|M+h\|^{H}-\|h\|^{H}=\|M\|^{H} .
$$

Setting $M=-h$ leads to a contradiction. There is indeed no self-similar graphs.

We will therefore turn to stochastic self-similarity, or statistical self-similarity as physicists say.

The conditions (3.1) and (3.2) are know rewritten in distribution. Let $\stackrel{(d)}{=}$ stands for equality of finite dimensional distributions.

(1) Invariance by isometry.

For any isometry of $\mathbb{R}^{n}$ and any $M \in \mathbb{R}^{n}$

$$
(X(i(M))-X(i(O)))_{M \in \mathbb{R}^{n}} \stackrel{(d)}{=}(X(M)-X(O))_{M \in \mathbb{R}^{n}} .
$$

(2) For all $\lambda \geq 0$,

$$
(X(\lambda M))_{M \in \mathbb{R}^{n}} \stackrel{(d)}{=} \lambda^{H}(X(M))_{M \in \mathbb{R}^{n}} .
$$

A field satisfying both (3.4) and (3.5) is called isotropic $H$-self-similar fields with strong stationary increments (in short $H$-sssis field) [76].

\subsection{Existence results}

A classification of $H$-sssis fields is not available yet. Nevertheless, the Gaussian and stable cases are rather well understood.

\subsubsection{Euclidean fractional Brownian fields}

$H$-sssis Gaussian fields lead to the celebrated fractional Brownian motion [23,62, 68, 85].

Theorem 3.1. There exists a unique, up to a multiplicative constant, $H$-sssis Gaussian centered field, iff $0<$ $H \leq 1$. Its covariance is given by

$$
\mathbb{E}(X(M) X(N))=C / 2\left[\|O M\|^{2 H}+\|O N\|^{2 H}-\|M N\|^{2 H}\right] .
$$

When $C=1$, this field is called a standard fractional Brownian motion. 
Remark 3.2. One classically speaks of fractional Brownian field. In order to avoid ambiguity, we will speak in this paper of Euclidean fractional Brownian fields to emphasize the fact that the set index is the Euclidean space.

Proof of Theorem 3.1. A computational Proof of Theorem 3.1, using the moving-average or harmonizable representations, is possible (e.g. [75]). Nevertheless this proof is non-natural, and we prefer the following proof.

Applying (3.5) with point $O$, one deduces $\mathbb{E} X^{2}(O)=0$ and $X(O)=0$ (a.s.). Applying (3.4) with translation and (3.5) leads to

$$
\begin{aligned}
\mathbb{E}(X(M)-X(N))^{2} & =\mathbb{E} X^{2}(M-N) \\
& =\|M N\|^{2 H} \mathbb{E} X^{2}((M-N) /\|M N\|) .
\end{aligned}
$$

Applying (3.5) with rotation proves that $\mathbb{E} X^{2}((M-N) /\|M N\|)$ is a constant $C$. Therefore, the covariance is given by

$$
\mathbb{E}(X(M) X(N))=C / 2\left[\|O M\|^{2 H}+\|O N\|^{2 H}-\|M N\|^{2 H}\right] .
$$

We now have to check whether this covariance function is indeed of positive type. By Theorem 2.2, we have to check that function $M, N \mapsto\|M N\|^{2 H}$ is of negative type. By Theorem 2.11, this is true iff $0<H \leq 1$.

\subsubsection{Stable fractional fields}

We assume the reader familiar with the stable fields, referring to [76]. Let us only recall that the characteristic function of a standard symmetric $\alpha$-stable variable $Z$ is given by

$$
\mathbb{E}\left(\mathrm{e}^{\mathrm{i} \lambda Z}\right)=\exp \left(-|\lambda|^{\alpha}\right)
$$

and that a field $X$ is a symmetric $\alpha$-stable if and only if all linear combination of $X$ are symmetric $\alpha$-stable variables. The stability index $\alpha$ is between 0 and 2, 2 being indeed the Gaussian case. With a slight abuse of language, one usually speaks of stable field for symmetric $\alpha$-stable field.

Unlike the Gaussian case, there is no uniqueness in the stable case. Several stable $H$-sssis can be built (e.g. [76]). We focus on the existence of stable $H$-sssis in terms of the fractional index $H$ and the stability index $\alpha[67,76]$.

Theorem 3.3. There exist stable $H$-sssis fields iff $0<H \leq 1 / \alpha$ if $0<\alpha<1$ and $0<H \leq 1$ if $1 \leq \alpha<2$.

Proof of Theorem 3.3. We refer indeed to [76].

(1) Since the restriction of a $H$-sssis field to the real line is a $H$-sssis process, it is sufficient to prove that $H$ and $\alpha$ must satisfy $0<H \leq 1 / \alpha$ if $0<\alpha<1$ and $0<H \leq 1$ if $1 \leq \alpha<2$ in dimension one. This is done by [76], Proposition 7.1.10.

(2) Then one needs to exhibit examples that cover the range of permissible index $H$ and $\alpha$. This is done in [76] Chapter 7, Section 8. Let us mention that, for $0<\alpha<2$ and $0<H<1$, this is done by integrating a random stable measure against a kernel, like an harmonizable or moving-average kernel. These kernels are presented in the section "Representations" of this paper. Please note that the one-dimensional process given in [76], Chapter 7 can be written in higher dimension by replacing the absolute value by a Euclidean norm. But this construction does not apply for $1<H<1 / \alpha$. This is done by constructing Chentsov-Takenaka fields. These construction indeed uses the fact that \|\|$^{\beta}, 0<\beta \leq 1$ are measure definite kernel distances. We will use later this construction. 


\subsection{General case}

\subsubsection{Generalities}

Self-similarity is based on scale invariance. Scale transformations are well defined in the Euclidean spaces, they are nothing else than a dilation. Unfortunately, scale invariance in a metric space or a Riemannian manifold is not well defined. For instance, there does not exist dilation in a compact metric space.

Let us be cautious. We don't claim that defining scale invariance is impossible. We only claim that there is not one canonical definition. We are using here two different ideas for a substitute to scale invariance.

(1) The invariance by zooming in and out is seminal. When one speaks of zoom, one implicitly refers to a underlying distance $d$. The minimal requirement seems then to impose the invariance of the increments, suitably normalized. In other words, we call a field $X(M)_{M \in E} H$-fractional, following [51,52], if the normalized increments $\frac{X(M)-X(N)}{d^{H}(M, N)}$ are constant in distribution. See section below.

(2) A second idea concerns the framework of smooth manifolds. We then use the exponential map to get the scale invariance from the tangent bundle onto the manifold. But this way of proceeding is of course local. See section below.

\subsubsection{Fractional Brownian fields}

We are looking for Gaussian fields which increments $\frac{X(M)-X(N)}{d^{H}(M, N)}$ are constant in distribution. There exists therefore a Gaussian variable $Z$ such that

$$
\frac{X(M)-X(N)}{d^{H}(M, N)} \stackrel{(d)}{=} Z
$$

Giving the distribution of the increments is not sufficient, we impose that, for a given point $O$,

$$
X(O)=0 \text { (a.s.). }
$$

Such a field is called a fractional Brownian field (indexed by $E$ ). Let us mention that, when $d$ is the Euclidean distance, definitions (3.6) and (3.7) fits with the Euclidean fractional Brownian field as defined in Theorem 3.1.

The question of existence then arises. The following Theorem shows that the Euclidean case is atypical.

Theorem 3.4. There exists a unique, up to a multiplicative constant, $H$-fractional Gaussian centered field, iff $0<H \leq \beta_{E} / 2$. Its covariance is given by

$$
\mathbb{E}(X(M) X(N))=C / 2\left[d^{2 H}(O, M)+d^{2 H}(O, N)-d^{2 H}(M, N)\right]
$$

When $C=1$, this field is called a standard fractional Brownian motion.

Proof of Theorem 3.4. Definitions (3.6) and (3.7) clearly lead to the covariance function

$$
\mathbb{E}(X(M) X(N))=C / 2\left[d^{2 H}(O, M)+d^{2 H}(O, N)-d^{2 H}(M, N)\right]
$$


We have therefore to check whether this covariance function is of positive type. By Theorem 2.2, we have to check that function $M, N \mapsto d(M, N)^{2 H}$ is of negative type. By Proposition 2.6, this is true iff $0<H \leq \beta_{E} / 2$.

Constructing covariance functions is not an easy task. The previous results provide us a way to construct several covariance functions related to Bernstein functions.

Proposition 3.5. Let $F$ be a Bernstein function with $F(0)=0$. The following functions are covariance functions

$$
\begin{aligned}
& R(M, N)=F\left(d^{\beta_{E} / 2}(O, M)\right)+F\left(d^{\beta_{E} / 2}(O, N)\right)-F\left(d^{\beta_{E} / 2}(M, N)\right), \\
& R(M, N)=\mathrm{e}^{-F\left(d^{\beta_{E} / 2}(M, N)\right)} .
\end{aligned}
$$

Examples of covariance functions with $0<H \leq \beta_{E} / 2$ are therefore

$$
\begin{aligned}
& R(M, N)=\log \left(1+\left(d^{2 H}(O, M)\right)+\log \left(1+\left(d^{2 H}(O, N)\right)-\log \left(1+d^{2 H}(M, N)\right),\right.\right. \\
& R(M, N)=\mathrm{e}^{-d^{2 H}(M, N)}, \\
& R(M, N)=\frac{1}{1+d^{2 H}(M, N)} .
\end{aligned}
$$

Proof of Proposition 3.5. Proposition 3.5 is indeed a direct consequence of Schönberg's Theorem 2.2, and Propositions 2.4 and 2.5 on Bernstein's functions.

\subsubsection{Stationary fields}

In a metric space, there is no notion of translation, unlike the case of a Euclidean space. The notion of stationarity has therefore to be adapted.

A field $X(M), M \in E$ is a field with stationary increments if the distribution of $X(M)-X(N)$ is a function of $d(M, N)$. A field $X(M), M \in E$ is stationary if it is with stationary increments and if the distribution of $X(M)$ does not depend on $M$.

One should wonder if there exists a stationary solution to (3.6). Although this question is very close to the stationary increments case, the answer is clearly different.

- $(E, d)$ unbounded. Let $X$ be a stationary field satisfying (3.6) and assume the existence of a moment of $\mathbb{E}|X(M)|^{\alpha}$ for an $\alpha>0$. One the one hand, since $(|a|+|b|)^{\alpha} \leq C_{\alpha}\left(|a|^{\alpha}+|b|^{\alpha}\right)$, it follows that $\mathbb{E} \mid X(M)-$ $\left.X(N)\right|^{\alpha} \leq 2 C_{\alpha} \mathbb{E}|X(M)|^{\alpha}$ and $\mathbb{E}|X(M)-X(N)|^{\alpha}$ is bounded. On the other hand, $\mathbb{E}|X(M)-X(N)|^{\alpha}=$ $\mathbb{E}|Z|^{\alpha} d^{\alpha H}(M, N)$ is clearly unbounded. There does not exist a stationary solution to (3.6) having an (arbitrary) small moment.

- $(E, d)$ bounded. The answer seems not to be known. Nevertheless, some remarks can be done. First, if $X$ is a Gaussian stationary field satisfying (3.6), then $B(M)=X(M)-X(O)$ is a fractional Brownian field. Therefore, index $H$ still satisfies $0<H \leq \beta_{E} / 2$. But all values between 0 and $\beta_{E} / 2$ are not necessarily permissible. Indeed, let $(E, d)=([0,1],||$.$) and consider the Gaussian stationary solution. Its covariance$ function is $(t, s) \mapsto 1-c|t-s|^{2 H}$ and is not of positive type for $H>1 / 2$.

\subsubsection{Set indexed fractional Brownian motion}

Assume that distance $d$ is a measure definite kernel as defined in Definition 2.7

$$
d(M, N)=\mu\left(H_{M} \Delta H_{N}\right) .
$$

By Proposition 2.8, $d$ is of negative type and $\beta_{E} \geq 1$. Since $d$ is a distance, the map $M \mapsto H_{M}$, defined in Definition 2.7, is injective. One can therefore define a standard fractional Brownian motion at least for $0<H \leq 1 / 2$ and its covariance function is given by

$$
\mathbb{E}(X(M) X(N))=\frac{1}{2}\left[\mu^{2 H}\left(H_{M} \Delta H_{O}\right)+\mu^{2 H}\left(H_{N} \Delta H_{O}\right)-\mu^{2 H}\left(H_{M} \Delta H_{N}\right)\right] .
$$


One can therefore consider that the field $X$ is indexed by the set $H_{M}$. Assume now that there exists a point $O$ such that $H_{O}=\emptyset$. This is for instance the case for $\left(\mathbb{R}^{n},\|\|\right)$ but not for $\left(\mathbb{S}^{n}, d\right)$. Then, the covariance is given by

$$
\mathbb{E}(X(M) X(N))=\frac{1}{2}\left[\mu^{2 H}\left(H_{M}\right)+\mu^{2 H}\left(H_{N}\right)-\mu^{2 H}\left(H_{M} \Delta H_{n}\right)\right],
$$

That is the way $[49,50]$ define and study the set indexed fractional Brownian motion. But, assuming $H_{O}=\emptyset$ seems to be an unnecessary condition.

\subsubsection{Stable fractional fields}

We are looking for stable fields which increments $\frac{X(M)-X(N)}{d^{H}(M, N)}$ are constant in distribution. There exists therefore a symmetric $\alpha$-stable variable $Z$ such that

$$
\begin{aligned}
\frac{X(M)-X(N)}{d^{H}(M, N)} & \stackrel{(d)}{=} Z \\
\mathbb{E}\left(\mathrm{e}^{\mathrm{i} \lambda Z}\right) & =\exp \left(-C|\lambda|^{\alpha}\right) .
\end{aligned}
$$

When $C=1$, we say that the symmetric stable variable $Z$ is standard. Giving the distribution of the increments is not sufficient, we impose that, for a given point $O$,

$$
X(O)=0 \text { (a.s.). }
$$

Such a field $X$ will be called an $H$-fractional $\alpha$-stable field. Please note that, for the sake of simplicity, we omit the word "symmetric" in the sequel. The question of existence then arises. The following theorem shows that, once again, the Euclidean case is atypical.

Theorem 3.6. Existence of $H$-fractional $\alpha$-stable fields.

- There is no $H$-fractional $\alpha$-stable field when $\alpha H>\beta_{E}$.

- There exists $H$-fractional $\alpha$-stable field for any $H$ and $\alpha$ providing $0<H \leq \beta_{E} / 2$.

- Assume moreover that distance $d$ is measure definite kernel. Then there exists $H$-fractional $\alpha$-stable field for any $H$ and $\alpha$ providing $1 / 2 \leq H \leq 1 / \alpha$.

- For spheres and hyperbolic spaces, there exists $H$-fractional $\alpha$-stable field iff $0<H \leq 1 / \alpha$.

Proof of Theorem 3.6.

(1) We prove the first item by contradiction. Let $\lambda, \lambda_{1}, \ldots, \lambda_{n} \in \mathbb{R}$ and $M_{1}, \ldots, M_{n} \in E$. On the one hand

$$
\sum_{i, j=1}^{n} \lambda_{i} \lambda_{j} \mathbb{E}\left[\exp \left(\mathrm{i} \lambda\left(X\left(M_{i}\right)-X\left(M_{j}\right)\right)\right)\right]=\mathbb{E}\left|\sum_{i=1}^{n} \lambda_{i} \exp \left(\mathrm{i} \lambda X\left(M_{i}\right)\right)\right|^{2} \geq 0 .
$$

On the other hand

$$
\sum_{i, j=1}^{n} \lambda_{i} \lambda_{j} \mathbb{E}\left[\exp \left(\mathrm{i} \lambda\left(X\left(M_{i}\right)-X\left(M_{j}\right)\right)\right)\right]=\sum_{i, j=1}^{n} \lambda_{i} \lambda_{j} \exp \left(-|\lambda|^{\alpha} d^{\alpha H}\left(M_{i}, M_{j}\right)\right)
$$

If $\alpha H>\beta_{E}$, Schönberg's Theorem 2.2 implies that there exists $\lambda$ such that $\exp \left(-|\lambda|^{\alpha} d^{\alpha H}(M, N)\right)$ is not of positive type and the item is proved. 
(2) The idea is to build a Poissonian sum of independent fractional Brownian fields. Indeed, we prove that the following formula for $n \geq 1, \lambda_{1}, \ldots, \lambda_{n} \in \mathbb{R}, M_{1}, \ldots, M_{n} \in E$,

$$
\mathbb{E}\left[\exp \left(i \sum_{j=1}^{n} \lambda_{j} X\left(M_{j}\right)\right)\right]=\exp \left[-\left|\sum_{i, j=1}^{n} \lambda_{i} \lambda_{j}\left(d^{2 H}\left(O, M_{i}\right)+d^{2 H}\left(O, M_{j}\right)-d^{2 H}\left(M_{i}, M_{j}\right)\right)\right|^{\alpha}\right]
$$

defines the distribution of an $H$-fractional $2 \alpha$-stable field $X(M), M \in E$. We indeed only need to prove that (3.12) defines the distribution of a stochastic field. We first need a preliminary lemma.

Let $\sigma$ be a positive measure on $[0,+\infty)$ satisfying

$$
\int_{0}^{+\infty} \inf (1, u) \sigma(\mathrm{d} u)<\infty
$$

Let $\phi: \mathbb{R}^{n} \rightarrow \mathbb{C}$ be a characteristic function such that there exist $C>0, \eta>0, \forall x \in \mathbb{S}^{1}, \forall u \in \mathbb{R}^{+}$

$$
\left|1-\phi\left(u^{\eta} x\right)\right| \leq C \inf (1, u)
$$

Lemma 3.7. Function $\Phi: \mathbb{R}^{n} \rightarrow \mathbb{C}$, defined by

$$
\Phi(x)=\exp \left(-\int_{0}^{+\infty}\left(1-\phi\left(u^{\eta} x\right)\right) \sigma(\mathrm{d} u)\right)
$$

is a characteristic function.

Proof of Lemma 3.7. We easily check that $\Phi(0)=1$. Function $x \mapsto 1-\phi\left(u^{\eta} x\right)$ is continuous and bounded by $C \inf (1, u)$. By the dominated convergence Theorem, $x \mapsto \Phi(x)$ is a continuous function.

We now prove that $x \mapsto \Phi(x)$ is of positive type. By Schoenberg's theorem, we have to check that function $x \mapsto \int_{0}^{+\infty}\left(1-\phi\left(u^{\eta} x\right)\right) \sigma(\mathrm{d} u)$ is of negative type. Take $\lambda_{1}, \ldots, \lambda_{p} \in \mathbb{R}$ with $\sum_{i=1}^{p} \lambda_{i}=0$ and $x_{1}, \ldots, x_{p} \in \mathbb{R}^{n}$

$$
\sum_{i, j=1}^{p} \lambda_{i} \lambda_{j} \int_{0}^{+\infty}\left(1-\phi\left(u^{\eta}\left(x_{i}-x_{j}\right)\right)\right) \sigma(\mathrm{d} u)=-\int_{0}^{+\infty} \sum_{i, j=1}^{p} \lambda_{i} \lambda_{j} \phi\left(u^{\eta}\left(x_{i}-x_{j}\right)\right) \sigma(\mathrm{d} u) .
$$

Since $\phi$ is a characteristic function, it is of positive type and $\sum_{i, j=1}^{p} \lambda_{i} \lambda_{j} \phi\left(u^{\eta}\left(x_{i}-x_{j}\right)\right) \geq 0$. It follows $\sum_{i, j=1}^{p} \lambda_{i} \lambda_{j}$ $\int_{0}^{+\infty}\left(1-\phi\left(u\left(x_{i}-x_{j}\right)\right)\right) \sigma(\mathrm{d} u) \leq 0$. By Bochner's theorem, $x \mapsto \Phi(x)$ is a characteristic function.

Let $\eta \leq 1 / \alpha$. Let $B$ be a fractional Brownian motion of index $0<H \leq \beta_{E} / 2$. Using the previous Lemma 3.7, the following formula, for $J \geq 1, \forall M_{1}, \ldots, M_{J} \in E, \forall \lambda_{1}, \ldots, \lambda_{J} \in \mathbb{R}$

$$
\mathbb{E}\left[\exp \left(\mathrm{i} \sum_{j=1}^{J} \lambda_{j} X\left(M_{j}\right)\right)\right]=\exp \left[-\int_{0}^{+\infty}\left(1-\mathbb{E}\left(\exp \left(\mathrm{i} u^{\eta} \sum_{j=1}^{J} \lambda_{j} B\left(M_{j}\right)\right)\right)\right) \sigma(\mathrm{d} u)\right]
$$


defines the distribution of a random field $X(M), M \in E$. One then checks that $\forall M, N \in E$

$$
\begin{aligned}
\mathbb{E}\left[\exp \left(\mathrm{i} \lambda \frac{X(M)-X(N)}{d^{H}(M, N)}\right)\right] & =\exp \left[-\int_{0}^{+\infty}\left(1-\mathbb{E}\left(\exp \left(\mathrm{i} u^{\eta} \lambda \frac{B(M)-B(N)}{d^{H}(M, N)}\right)\right)\right) \sigma(\mathrm{d} u)\right] \\
& =\exp \left[-\int_{0}^{+\infty}\left(1-\mathbb{E}\left(\exp \left(\mathrm{i} u^{\eta} \lambda Z\right)\right)\right) \sigma(\mathrm{d} u)\right]
\end{aligned}
$$

where $Z$ is a standard Gaussian variable. Let now

$$
\sigma(\mathrm{d} u)=4^{\alpha}\left(\int_{0}^{+\infty} \frac{1-\mathrm{e}^{-v}}{v^{1+\alpha}} \mathrm{d} v\right)^{-1} \frac{\mathrm{d} u}{u^{1+\alpha}}, \quad 0<\alpha<1,
$$

and take $\eta=1 / 2$. Then

$$
\mathbb{E}\left[\exp \left(\mathrm{i} \lambda \frac{X(M)-X(N)}{d^{H}(M, N)}\right)\right]=\exp \left(-|\lambda|^{2 \alpha}\right)
$$

and the field $X$ is $H$-fractional $2 \alpha$-stable.

(3) Recall that distance $d$ is a measure definite kernel if there exists a measure space $(\mathbf{H}, \sigma(\mathbf{H}), \mu)$ and a map $M \mapsto H_{M}$ from $E$ onto $\sigma(\mathbf{H})$ such that

$$
d(M, N)=\mu\left(H_{M} \Delta H_{N}\right)
$$

where $\Delta$ denotes the symmetric difference of sets.

For $\beta>0, f \in L^{\beta}(\mathbf{H}, \mu)$, define the pseudo-norm

$$
\|f\|_{\beta}=\left(\int_{\mathbf{H}}|f|^{\beta} \mathrm{d} \mu\right)^{1 / \beta} .
$$

It follows that

$$
\begin{aligned}
d(M, N) & =\int_{\mathbf{H}}\left|\mathbf{1}_{H_{M}}-\mathbf{1}_{H_{N}}\right| \mathrm{d} \mu \\
& =\left\|\mathbf{1}_{H_{M}}-\mathbf{1}_{H_{N}}\right\|_{\beta}^{\beta} .
\end{aligned}
$$

Let $1 / 2 \leq H \leq 1 / \alpha$. We prove that the following formula, with $n \geq 1, \lambda_{1}, \ldots, \lambda_{n} \in \mathbb{R}, M_{1}, \ldots, M_{n} \in E$,

$$
\mathbb{E}\left(\exp \left(i \sum_{j=1}^{n} \lambda_{j} X\left(M_{j}\right)\right)\right)=\exp \left(-\left\|\sum_{j=1}^{n} \lambda_{j} \mathbf{1}_{H_{M_{j}}}\right\|_{1 / H}^{\alpha}\right)
$$

defines the distribution of an $\alpha$-stable field $X(M), M \in E$. We follow Theorem 1 and Lemma 4 of [20]. We have seen that function $(x, y) \mapsto|x-y|^{\gamma}, x, y \in \mathbb{R}$ is of negative type if $0<\gamma \leq 2$. It follows that function $(f, g) \mapsto\|f-g\|_{1 / H}^{1 / H}, f, g \in L^{1 / H}(\mathbf{H}, \mu)$ is of negative type when $H \geq 1 / 2$. Since $\alpha H \leq 1$, one can apply Propositions 2.4 and 2.5: function $(f, g) \mapsto\|f-g\|_{1 / H}^{\alpha}, f, g \in L^{1 / H}(\mathbf{H}, \mu)$ is of negative type. Schoenberg's Theorem 2.2 implies that, for all $\lambda \in \mathbb{R}$, function $(f, g) \mapsto \exp \left(-|\lambda|^{\alpha}|| f-g \|_{1 / H}^{\alpha}\right), f, g \in L^{1 / H}(\mathbf{H}, \mu)$ is of positive type. (3.14) is therefore a characteristic function. The Kolmogorov consistency theorem then proves that (3.14) defines the distribution of an $\alpha$-stable stochastic fields.

Choosing $n=2$ and $\lambda_{1}=-\lambda_{2}$ in (3.14), and using (3.13), shows that $X$ is $H$-fractional $\alpha$-stable. 
Remark 3.8. One could wonder if function $(f, g) \mapsto\|f-g\|_{1 / H}^{\alpha}$ is always of negative type for $H<1 / 2$. Assume that we can choose three disjoints sets $A_{1}, A_{2}$ and $A_{3}$ such that $\mu\left(A_{1}\right)=\mu\left(A_{2}\right)=\mu\left(A_{3}\right)=c>0$.

Put $f=\sum_{1}^{3} \lambda_{i} \mathbf{1}_{A_{i}}$. Then

$$
\|f\|_{1 / H}^{\alpha}=\mathrm{c}^{\alpha H}\left(\sum_{1}^{3}\left|\lambda_{i}\right|^{1 / H}\right)^{\alpha H} .
$$

But one knows [60,61] that function $(x, y) \mapsto\|x-y\|_{\ell q}^{p}, x, y \in \mathbb{R}^{n}$ is never of negative type when $n \geq 3$, $0<p \leq 2$ and $q>2$. Function $(f, g) \mapsto\|f-g\|_{1 / H}^{\alpha}$ is not of negative type for $H<1 / 2$ in this example.

(4) The fourth item follows directly from the three first items of Theorem 3.6 and Theorems 2.13 and 2.14.

\section{REPRESENTATIONS}

We give in this section representations of Gaussian and stable fields and we refer to [32] for detailed results and proofs. We assume the reader familiar with the concept of Gaussian or stable random measure, and refer to $[76]$.

\subsection{Euclidean case}

One wishes to represent the Euclidean fractional Brownian motion as a random integral or a random series. Even if the result is well-known, we describe here a way to get it. One starts from Lévy-Khintchine's formula. Indeed, since function $\|M N\|^{2 H}$ is of negative type, there exists a quadratic form $Q$ and an unique positive symmetric measure $\mu$ such that

$$
\|O M\|^{2 H}=Q(O M)+\int_{\mathbb{R}^{n}}\left(1-\mathrm{e}^{\mathrm{i}\langle O M, \Lambda\rangle}\right) \mu(\mathrm{d} \Lambda) .
$$

When $H=1, \mu=0$ and $Q(O M)=\|O M\|^{2}$. Assume now $0<H<1$. Assume provisionally that $Q=0$ and that measure $\mu$ admits a density

$$
\mu(\mathrm{d} \Lambda)=f(\Lambda) \mathrm{d} \Lambda
$$

Let $\lambda>0$. On the one hand

$$
\|\lambda O M\|^{2 H}=\lambda^{2 H}\|O M\|^{2 H},
$$

on the other, using the change of variable $\widetilde{\Lambda}=\lambda \Lambda$

$$
\|\lambda O M\|^{2 H}=\int_{\mathbb{R}^{n}}\left(1-\mathrm{e}^{\mathrm{i}\langle O M, \widetilde{\Lambda}\rangle}\right) f(\widetilde{\Lambda} / \lambda) \frac{d \widetilde{\Lambda}}{\lambda^{n}} .
$$

This leads to, for all $\widetilde{\Lambda}$

$$
f(\widetilde{\Lambda})=\frac{f(\widetilde{\Lambda} / \lambda)}{\lambda^{n+2 H}} .
$$

Let now $R$ be an arbitrary rotation of $\mathbb{R}^{n}$ of center $O$. One the one hand

$$
\|\lambda O R(M)\|^{2 H}=\|O M\|^{2 H},
$$


on the other, using the change of variable $\widetilde{\Lambda}=R^{-1} \Lambda$

$$
\|\lambda O R(M)\|^{2 H}=\int_{\mathbb{R}^{n}}\left(1-\mathrm{e}^{\mathrm{i}\langle O M, \widetilde{\Lambda}\rangle}\right) f(R \widetilde{\Lambda}) \mathrm{d} \widetilde{\Lambda} .
$$

Basic computations shows that $c$ is finite as soon as $0<H<1$. This leads to

$$
f(R \widetilde{\Lambda})=f(\widetilde{\Lambda})
$$

By combining both (4.1) and (4.2)

$$
f(\Lambda)=\frac{2 c}{\|\Lambda\|^{n+2 H}}
$$

where

$$
c=\frac{1}{2}\left(\int_{\mathbb{R}^{n}} \frac{1-\mathrm{e}^{\mathrm{i}\langle\mathbf{1}, \boldsymbol{\Lambda}\rangle}}{\|\Lambda\|^{n+2 H}}\right)^{-1} \mathrm{~d} \Lambda .
$$

The covariance function of the standard Euclidean fractional Brownian motion can therefore be written as

$$
\frac{1}{2}\left(\|O M\|^{2 H}+\|O N\|^{2 H}-\|M N\|^{2 H}\right)=c \int_{\mathbb{R}^{n}} \frac{\left(1-\mathrm{e}^{\mathrm{i}\langle O M, \Lambda\rangle)}\right.}{\|\Lambda\|^{n / 2+H}} \frac{\left(1-\mathrm{e}^{-\mathrm{i}\langle O N, \Lambda\rangle)}\right.}{\|\Lambda\|^{n / 2+H}} \mathrm{~d} \Lambda .
$$

In other words, the standard Euclidean fractional Brownian motion can be represented as a stochastic integral

$$
X(M) \stackrel{(d)}{=} c \int_{\mathbb{R}^{n}} \frac{1-\mathrm{e}^{\mathrm{i}\langle O M, \Lambda\rangle}}{\|\Lambda\|^{n / 2+H}} \mathrm{~d} \hat{W}(\Lambda),
$$

where $\hat{W}$ is the Fourier transform of a random Brownian measure on $L^{2}\left(\mathbb{R}^{n}\right)$. Let $I$ be an isometry of $L^{2}\left(\mathbb{R}^{n}\right)$. $X$ then admits the representation

$$
X(M) \stackrel{(d)}{=} c \int_{\mathbb{R}^{n}} I\left(\frac{1-\mathrm{e}^{\mathrm{i}\langle O M, \Lambda\rangle}}{\|\Lambda\|^{n / 2+H}}\right) \mathrm{d} W(\Lambda) .
$$

In particular, when $I$ is the Fourier transform, this leads to the moving-average representation [66]

$$
X(M) \stackrel{(d)}{=} c^{\prime} \int_{\mathbb{R}^{n}}\left(\|O M-\Lambda\|^{n / 2+H}-\|\Lambda\|^{n / 2+H}\right) \mathrm{d} W(\Lambda)
$$

The previous representations are only equality in distribution. More precise results, including (a.s.) equality, are given in [31].

Let now $e_{n}, n \geq 0$ be an orthonormal basis of $L^{2}\left(\mathbb{R}^{n}\right)$. Since $\frac{1-\mathrm{e}^{\mathrm{i}\langle O M, \Lambda\rangle}}{\|\Lambda\|^{n / 2+H}}$ belongs to $L^{2}\left(\mathbb{R}^{n}\right)$

$$
c \frac{1-\mathrm{e}^{\mathrm{i}\langle O M, \Lambda\rangle}}{\|\Lambda\|^{n / 2+H}}=\sum_{n \geq 0} f_{n}(M) e_{n}(\Lambda)
$$

and $X$ can be represented as a random series

$$
X(M) \stackrel{(d)}{=} \sum_{n \geq 0} f_{n}(M) e_{n}(\Lambda) \varepsilon_{n}
$$


where $\varepsilon_{n}$ is a sequence of i.i.d. standard Gaussian variables. Note that these representations in distribution are, with suitable conditions, almost surely equality [29].

Let us mention now that the Gaussian harmonizable and moving-average representation have a stable counterpart [76]. Indeed, the two following fields are $H$ self-similar with stationary increments

$$
\begin{aligned}
& Y(M) \stackrel{(d)}{=} \int_{\mathbb{R}^{n}} \frac{1-\mathrm{e}^{\mathrm{i}\langle O M, \Lambda\rangle}}{\|\Lambda\|^{n / \alpha+H}} \mathrm{~d} W_{\alpha}^{1}(\Lambda), \\
& Z(M) \stackrel{(d)}{=} \int_{\mathbb{R}^{n}}\left(\|O M-\Lambda\|^{n / \alpha+H}-\|\Lambda\|^{n / \alpha+H}\right) \mathrm{d} W_{\alpha}^{2}(\Lambda),
\end{aligned}
$$

where $W_{\alpha}^{1}$ and $W_{\alpha}^{2}$ are random stable measures.

\subsection{Spherical case}

\subsubsection{Mercer's theorem}

Since the sphere is compact, we will use the Mercer's theorem [86] that we remember now.

Suppose that $k$ is a continuous symmetric kernel on a compact set $X$ and that the integral operator $T$, $L^{2}(X, \mathrm{~d} x) \rightarrow L^{2}(X, \mathrm{~d} x)$ defined by

$$
T f(.)=\int_{X} k(., x) f(x) \mathrm{d} x
$$

is positive semidefinite, i.e.

$$
\int_{X} k(x, y) f(y) f(x) \mathrm{d} x \mathrm{~d} y \geq 0 .
$$

Then there is an orthonormal basis $\left(\psi_{n}\right)$ of $L^{2}(X, \mathrm{~d} x)$ consisting of eigenfunctions of $T$, with corresponding non-negative eignevalues $\left(\lambda_{n}\right) . k$ has the representation

$$
k(x, y)=\sum_{n \geq 0} \lambda_{n} \psi_{n}(x) \psi_{n}(y)
$$

where the convergence is absolute and uniform.

\subsubsection{Expansions}

We now give the harmonizable and moving-average representations of the spherical fractional Brownian motion [53]. We should use the Lévy-Khintchine's formula in the spherical case (e.g. [44]), but a direct computation is possible and we do it. The Gegenbauer polynomials $[40,78,80]$, also known as ultraspherical polynomials, are defined via the Rodrigue's formula

$$
P_{\ell}^{(\lambda)}(x)=\frac{(-1)^{\ell}}{2^{\ell} \ell !} \frac{\Gamma(\lambda+1 / 2) \Gamma(\ell+2 \lambda)}{\Gamma(2 \lambda) \Gamma(\lambda+\ell+1 / 2)\left(1-x^{2}\right)^{\lambda-1 / 2}} \frac{\mathrm{d}^{\ell}}{\mathrm{d} x^{\ell}}\left[\left(1-x^{2}\right)^{\lambda-1 / 2}\left(1-x^{2}\right)\right] .
$$

They satisfy $P_{\ell}^{(\lambda)}(1)=\left(\begin{array}{c}\ell+2 \lambda \\ \ell\end{array}\right)$. The spherical harmonics ([40], Chap. 11, [78]) are the eigenfunctions of the Laplacian on $\mathbb{S}^{n}$. Let $S H_{\ell}=\left\{S_{m, \ell}, m=1, \ldots, h(\ell)\right\}$ denotes the set of all spherical harmonics of degree $\ell$, then the dimension of $S H_{\ell}$ is

$$
h(\ell)=\frac{2 \ell+n-1}{\ell+n-1}\left(\begin{array}{c}
\ell+n-1 \\
\ell
\end{array}\right)
$$


We get the direct sum decomposition

$$
L^{2}\left(\mathbb{S}^{n}\right)=\bigoplus_{\ell \geq 0} S H_{\ell}
$$

The spherical addition theorem, also known as the Legendre addition theorem $[78,80]$, is given by

$$
\frac{1}{h(\ell)} \sum_{m=1}^{h(\ell)} S_{\ell, m}(M) \overline{S_{\ell, m}\left(M^{\prime}\right)}=\frac{P_{\ell}^{(\lambda)}\left(\overrightarrow{C M} \overrightarrow{C M^{\prime}}\right)}{P_{\ell}^{(\lambda)}(1)},
$$

where $C$ is the center of the sphere and $\lambda=n / 2-1$.

We then get the harmonizable expansion of the spherical fractional Brownian motion.

$$
X(M) \stackrel{L^{2},(\text { a.s. })}{=} \sum_{\ell \geq 0} \sum_{m=1}^{h(\ell)} \sqrt{-x_{\ell} P^{(\lambda)}(1) / h(\ell)} \varepsilon_{m, \ell}\left(S_{\ell, m}(M)-S_{\ell, m}(O)\right) \text {, }
$$

where the $\left(\varepsilon_{m, \ell}\right)$ are i.i.d. centered standard normal variables and the $\left(x_{\ell}\right)$ are given by

$$
\arccos ^{2 H}(x)=\sum_{\ell \geq 0} x_{\ell} P_{\ell}^{(\lambda)}(x)
$$

Let us now prove expansion (4.3). Let $R$ be the linear operator

$$
R \psi(M)=\int_{\mathbb{S}_{n}} \psi\left(M^{\prime}\right) d^{2 H}\left(M, M^{\prime}\right) \mathrm{d} \sigma\left(M^{\prime}\right) .
$$

By the spherical addition theorem, the spherical harmonics are eigenfunctions of $R$

$$
\begin{aligned}
\int_{\mathbb{S}_{n}} S_{m, \ell}\left(M^{\prime}\right) \mathrm{d}^{2 H}\left(M, M^{\prime}\right) \mathrm{d} \sigma\left(M^{\prime}\right) & =\int_{\mathbb{S}_{n}} S_{m, \ell}\left(M^{\prime}\right) \arccos ^{2 H}\left(\overrightarrow{C M} \overrightarrow{C M^{\prime}}\right) \mathrm{d} \sigma\left(M^{\prime}\right) \\
& =\sum_{\ell \geq 0} x_{\ell} \int_{\mathbb{S}_{n}} S_{m, \ell}\left(M^{\prime}\right) P_{\ell}^{(\lambda)}\left(\overrightarrow{C M} \overrightarrow{C M^{\prime}}\right) \mathrm{d} \sigma\left(M^{\prime}\right) \\
& =x_{\ell} P_{\ell}^{(\lambda)}(1) / h(\ell) S_{m, \ell}(M) .
\end{aligned}
$$

Since $H \leq 1 / 2$, the eigenvalues are non-negative and $R$ is a non-negative definite operator. $R$ is clearly symmetric. By Mercer's theorem, a symmetric non-negative definite kernel can be expanded on its eigenfunctions. Moreover, since the kernel $R$ is continuous, the expansion hold $L^{2}$ and (a.s.). The harmonizable expansion of $X$ is proved.

Let now define the following kernel

$$
K(M, P)=\sum_{\ell \geq 0} \sum_{m=1}^{h(\ell)} \sqrt{-x_{\ell} P^{(\lambda)}(1) / h(\ell)} S_{\ell, m}(M) S_{\ell, m}(P) .
$$

Thanks to the spherical addition theorem, $K(M, P)$ depends only on the geodesic distance $d(M, P)$. $X$ then admits the moving-average representation

$$
X(M) \stackrel{L^{2}, \text { (a.s.) }}{=} \int_{\mathbb{S}^{n}}(K(M, P)-K(O, P)) \mathrm{d} W(P),
$$


where $W$ is a random Brownian measure on $L^{2}\left(\mathbb{S}^{n}, \mathrm{~d} \sigma\right)$. To find a simple analytic expression of $K$ is an open problem.

\subsection{Hyperbolic case}

Most of the paper [32] is devoted to the spectral analysis of the hyperbolic case. Let us briefly mention the particular problem of the hyperbolic fractional Brownian motion. One aims, as in the Euclidean case, to get a harmonizable representation. One starts too from the Lévy-Khintchine's formula [44]. For $H=1 / 2$, the representation is given in [32]. For $0<H<1 / 2$, since $d^{2 H}$ is of negative type, one knows that there exists a positive measure $\mu$ such that, for all $z \in \mathbb{H}^{n}$

$$
d^{2 H}(O, z)=\int\left(1-\phi_{\lambda}(z)\right) \mu(\mathrm{d} \lambda)
$$

where $\phi_{\lambda}$ is the eigenfunction, associated to the eigenvalue $\lambda$, of the hyperbolic Laplace-Beltrami operator. The hyperbolic fractional Brownian motion therefore admits $\mu$ as spectral measure. We refer to [32] to know whether this measure $\mu$ admits a density or not.

\section{LOCAL SELF-SIMILARITY}

\subsection{Euclidean case}

Let us recall that a field $X$ is self-similar with index $H$ if

$$
(X(\lambda x))_{x \in \mathbb{R}^{n}} \stackrel{(d)}{=} \lambda^{H}(X(x))_{x \in \mathbb{R}^{n}} .
$$

One now aims to localize this property. This has been introduced since a while by [38] and rediscovered by $[8,69]$. There is now no reason, even if one usually does, to restrict to fractional power for the scale function c. A field $X$ is locally asymptotically self-similar (in short lass) with scaling function $c$ at point $x_{0}$ if there exists a non-degenerate ${ }^{3}$ tangent field $Z$ such that

$$
\lim _{\varepsilon \rightarrow 0^{+}}\left(\frac{X\left(x_{0}+\varepsilon x\right)-X\left(x_{0}\right)}{c(\varepsilon)}\right)_{x \in \mathbb{R}^{n}} \stackrel{(d)}{=}(Z(x))_{x \in \mathbb{R}^{n}} .
$$

A field $X$ is locally asymptotically self-similar with index $H$ if $c(\varepsilon)=\varepsilon^{H}$. If a field $X$ is self-similar with index $H$, then $X$ is lass with index $H$ at point $O$ with itself as tangent field. If a field $X$, satisfying $X(O)=0$ (a.s.), is $H$-sssis, then $X$ is lass with index $H$ at every point with itself as tangent field. The property of the tangent fields has been investigated by [41,42]. Let us only mention, that, roughly speaking, the tangent field is, almost everywhere, self-similar with stationary increments. In particular, if the tangent field is Gaussian, then it is an Euclidean fractional Brownian field.

\subsection{Manifold case}

\subsubsection{Definition}

We present in this section the approach of [56]. Let us recall some notations. $\mathcal{M}$ is our manifold. Let $M_{0} \in \mathcal{M}$. $T_{M_{0}} \mathcal{M}$ is the tangent space to $\mathcal{M}$ at $M_{0}$. Then, there exists a neighborhood $\mathcal{V}\left(M_{0}\right)$ of $M_{0}$ and $\delta>0$, see for example $[45,70]$, such that

(1) for all $M \in \mathcal{V}\left(M_{0}\right)$, there exists a unique minimal geodesic between $M$ and $M_{0}$;

(2) the exponential map $\exp _{M_{0}}$ at point $M_{0}$ is a diffeomorphism between the open ball $\mathcal{B}(0, \delta) \subset T_{M_{0}} \mathcal{M}$ and $\mathcal{V}\left(M_{0}\right)$

\footnotetext{
${ }^{3}$ The precise definition of a non-degenerate field depend on the context.
} 
(3) for any $v, w \in \mathcal{B}(0, \delta)$ (e.g. [70], Chap. 5),

$$
\lim _{\varepsilon \rightarrow 0^{+}} \frac{d\left(\exp _{M_{0}}(\varepsilon v), \exp _{M_{0}}(\varepsilon w)\right)}{\varepsilon}=\|v-w\| .
$$

We extend the lass notion to fields indexed by a manifold which is not in general a vector space. Hence, we first have to interpret $x_{0}+\varepsilon x$ as a point of the manifold $\mathbb{R}^{n}$ without the help of the addition on $\mathbb{R}^{n}$. Note that the tangent space to $\mathbb{R}^{n}$ at any point is identified to $\mathbb{R}^{n}$. On the one hand, $x_{0}$ is a point of $\mathbb{R}^{n}$, which corresponds to the point $M_{0}$ for the manifold $\mathcal{M}$. On the other hand, $x_{0}+\varepsilon x$ is the shift of $M_{0}$ by the vector $\varepsilon x \in T_{x_{0}} \mathbb{R}^{n} \approx \mathbb{R}^{n}$. Also, since the geodesics in $\mathbb{R}^{n}$ are the segments, we have

$$
x_{0}+\varepsilon x=\exp _{x_{0}}(\varepsilon x) .
$$

Then, we propose to replace in (5.1) the point $x_{0}$ by $M_{0}$ and its translate $x_{0}+\varepsilon x$ by

$$
M_{0}+\varepsilon v \stackrel{\text { def }}{=} \exp _{M_{0}}(\varepsilon v)
$$

Note that $M_{0}+\varepsilon v$ is well defined as soon as $v \in \mathcal{B}(0, \delta)$ and $\varepsilon \in[0,1]$. Let us fix $M \in \mathcal{V}\left(M_{0}\right)$. Then, there exists a unique $v \in \mathcal{B}(0, \delta)$ such that $\exp _{M_{0}}(v)=M$ so that $M_{0}+v=M$. Moreover, $M_{0}+\varepsilon v$ is the only point of the geodesic between $M_{0}$ and $M$ such that

$$
d\left(M_{0}, M_{0}+\varepsilon M\right)=\varepsilon d\left(M_{0}, M\right) .
$$

The range of $M_{0}+\varepsilon v$ is the geodesic between $M_{0}$ and $M$ as $\varepsilon$ varies in [0,1]. In addition, as $\varepsilon$ tends to zero, $M_{0}+\varepsilon v$ tends to $M_{0}$ in the direction given by this geodesic.

Definition 5.1. $X=(X(M))_{M \in \mathcal{M}}$ is locally asymptotically self-similar (lass in short) at point $M_{0}$ with index $h\left(M_{0}\right)>0$ if

$$
\lim _{\varepsilon \rightarrow 0^{+}}\left(\frac{X\left(M_{0}+\varepsilon v\right)-X\left(M_{0}\right)}{\varepsilon^{h\left(M_{0}\right)}}\right)_{v \in B(0, \delta)} \stackrel{(d)}{=}\left(Z_{M_{0}}(v)\right)_{v \in B(0, \delta)}
$$

where $Z_{M_{0}}$ is a non degenerate field, that is $\exists u$, such that

$$
\mathbb{P}\left(Z_{M_{0}}(u) \neq 0\right)=1,
$$

and $\stackrel{(d)}{=}$ stands for equality of finite dimensional distributions. $Z_{M_{0}}$ is called the tangent field at point $M_{0}$ of $X$.

As one could expect, the definition of a lass random field at point $M_{0}$ and the definition of its tangent field coincide with the Euclidean definitions in the framework of Euclidean random fields.

\subsubsection{Weak stationarity}

We extend now the weak stationarity in the framework of manifold indexed fields.

Definition 5.2. The increments of the field $X=(X(M))_{M \in \mathcal{M}}$ are weakly stationary if for all $(M, N) \in \mathcal{M}^{2}$, the distribution of $X(M)-X(N)$ only depends on the geodesic distance $d(M, N)$. More precisely, there exists a function $\psi$ such that for all $(M, N) \in \mathcal{M}^{2}$ and all $\lambda \in \mathbb{R}$,

$$
\mathbb{E}\left[\mathrm{e}^{\mathrm{i} \lambda(X(M)-X(N))}\right]=\psi(\lambda, d(M, N)) .
$$




\subsubsection{Properties}

Theorem 5.3. If $X$ is lass at point $M_{0}$ with index $h\left(M_{0}\right)$ and tangent field $Z_{M_{0}}$, then $Z_{M_{0}}(0)=0$ almost surely and

$$
\forall \lambda \in(0,1],\left(Z_{M_{0}}(\lambda v)\right)_{v \in B(0, \delta)} \stackrel{(d)}{=} \lambda^{h\left(M_{0}\right)}\left(Z_{M_{0}}(v)\right)_{v \in B(0, \delta)} .
$$

Moreover, if $X$ has weakly stationary increments so has $Z_{M_{0}}$.

Corollary 5.4. Let $X$ be a lass random field at point $M_{0}$ with index $h\left(M_{0}\right)$ and tangent field $Z_{M_{0}}$. Assume that $X$ has weakly stationary increments and that for some $u \in \mathcal{B}(0, \delta) \backslash\{0\}$ and some $\gamma>0$,

$$
\mathbb{P}\left(Z_{M_{0}}(u) \neq 0\right)=1 \quad \text { and } \quad \mathbb{E}\left|Z_{M_{0}}(u)\right|^{\gamma}<+\infty .
$$

(1) If $0<\gamma<1$, then $0<h\left(M_{0}\right)<\frac{1}{\gamma}$.

(2) If $\gamma \geq 1$, then $0<h\left(M_{0}\right) \leq 1$.

Corollary 5.5. Assume that $X$ has weakly stationary increments and is lass at point $M_{0}$ with index $h\left(M_{0}\right)$ and tangent field $Z_{M_{0}}$.

(1) If $Z_{M_{0}}$ is a centered Gaussian random field, then

(a) $h\left(M_{0}\right) \in(0,1]$;

(b) there exists a constant $C>0$ such that the covariance function of $Z_{M_{0}}$ is given by

$$
\forall v, w \in \mathcal{B}(0, \delta), \mathbb{E}\left(Z_{M_{0}}(v) Z_{M_{0}}(w)\right)=\frac{C^{2}}{2}\left(\|v\|^{2 h\left(M_{0}\right)}+\|w\|^{2 h\left(M_{0}\right)}-\|v-w\|^{2 h\left(M_{0}\right)}\right) .
$$

Moreover, $Z_{M_{0}}$ is an Euclidean fractional Brownian motion restricted to $B(0, \delta)$.

(2) If $Z_{M_{0}}$ is an $\alpha$-stable random field, then $0<h\left(M_{0}\right) \leq \max \left(1, \frac{1}{\alpha}\right)$.

Proof of Theorem 5.3. Since for $v=0, M_{0}+\varepsilon v=M_{0}$, it is straightforward that $Z_{M_{0}}(0)=0$ almost surely. Let $\lambda \in(0,1]$. Then, by definition

$$
\begin{aligned}
& \left(Z_{M_{0}}(\lambda v)\right)_{v \in B(0, \delta)} \stackrel{(d)}{=} \lim _{\varepsilon \rightarrow 0^{+}}\left(\frac{X\left(M_{0}+\varepsilon \lambda v\right)-X\left(M_{0}\right)}{\varepsilon^{h\left(M_{0}\right)}}\right)_{v \in B(0, \delta)} \\
& \stackrel{(d)}{=} \lambda^{h\left(M_{0}\right)} \lim _{\varepsilon \rightarrow 0^{+}}\left(\frac{X\left(M_{0}+\varepsilon \lambda v\right)-X\left(M_{0}\right)}{(\lambda \varepsilon)^{h\left(M_{0}\right)}}\right)_{v \in B(0, \delta)} \\
& \stackrel{(d)}{=} \lambda^{h\left(M_{0}\right)}\left(Z_{M_{0}}(\lambda v)\right)_{v \in B(0, \delta)} .
\end{aligned}
$$

Let us now assume that $X$ has weakly stationary increments. Let $v, w \in \mathcal{B}(0, \delta)$. Then, by the lass property

$$
Z_{M_{0}}(v)-Z_{M_{0}}(w) \stackrel{(d)}{=} \lim _{\varepsilon \rightarrow 0^{+}} \frac{X\left(M_{0}+\varepsilon v\right)-X\left(M_{0}+\varepsilon w\right)}{\varepsilon^{h\left(M_{0}\right)}} .
$$

Let us fix $u \in \mathcal{B}(0, \delta)$ such that $u \neq 0$. We recall that $M_{0}+\varepsilon v=\exp _{M_{0}}(v)$ and that $M_{0}+\varepsilon w=\exp _{M_{0}}(w)$. Then, by continuity of the distance and of the exponential map $\exp _{M_{0}}$, there exists $\varepsilon_{0}>0$ such that

$$
\forall \varepsilon \leq \varepsilon_{0}, d\left(M_{0}+\varepsilon v, M_{0}+\varepsilon w\right)<\|u\| .
$$

Therefore, the point

$$
M_{0}+\frac{d\left(M_{0}+\varepsilon v, M_{0}+\varepsilon w\right)}{\|u\|} u=\exp _{M_{0}}\left(\frac{d\left(M_{0}+\varepsilon v, M_{0}+\varepsilon w\right)}{\|u\|} u\right)
$$


is well defined. By construction,

$$
d\left(M_{0}, M_{0}+\frac{d\left(M_{0}+\varepsilon v, M_{0}+\varepsilon w\right)}{\|u\|} u\right)=d\left(M_{0}+\varepsilon v, M_{0}+\varepsilon w\right) .
$$

Hence, by (5.5) and by the weakly stationarity of the increments of $X$,

$$
Z_{M_{0}}(v)-Z_{M_{0}}(w) \stackrel{(d)}{=} \lim _{\varepsilon \rightarrow 0^{+}} \frac{X\left(M_{0}+\frac{d\left(M_{0}+\varepsilon v, M_{0}+\varepsilon v\right)}{\|u\|} u\right)-X\left(M_{0}\right)}{\varepsilon^{h\left(M_{0}\right)}} .
$$

Applying the lass property, we then have that

$$
Z_{M_{0}}(v)-Z_{M_{0}}(w) \stackrel{(d)}{=}\|u\|^{-h\left(M_{0}\right)} Z_{M_{0}}(u) \lim _{\varepsilon \rightarrow 0^{+}} \frac{d\left(M_{0}+\varepsilon v, M_{0}+\varepsilon w\right)^{h\left(M_{0}\right)}}{\varepsilon^{h\left(M_{0}\right)}} .
$$

For any $v, w \in \mathcal{B}(0, \delta)$,

$$
\lim _{\varepsilon \rightarrow 0^{+}} \frac{d\left(M_{0}+\varepsilon v, M_{0}+\varepsilon w\right)}{\varepsilon}=\|v-w\| .
$$

This leads to

$$
Z_{M_{0}}(v)-Z_{M_{0}}(w) \stackrel{(d)}{=}\|u\|^{-h\left(M_{0}\right)}\|v-w\|^{h\left(M_{0}\right)} Z_{M_{0}}(u)
$$

for any $v, w \in \mathcal{B}(0, \delta)$, which establishes that the increments of $Z_{M_{0}}$ are weakly stationary.

Proof of Corollary 5.4. By the proof of Theorem 5.3,

$$
\forall v \in \mathcal{B}(0, \delta), Z_{M_{0}}(v) \stackrel{(d)}{=}\|v\|^{h\left(M_{0}\right)}\|u\|^{-h\left(M_{0}\right)} Z_{M_{0}}(u)
$$

Since $Z_{M_{0}}(u) \neq 0$ almost surely,

$$
\mathbb{E}\left|Z_{M_{0}}(u)\right|^{\gamma} \neq 0
$$

Moreover, by (5.6), for every $v \in \mathcal{B}(0, \delta) \backslash\{0\}$

$$
Z_{M_{0}}(v) \neq 0 \text { almost surely. }
$$

Furthermore, let us remark that $u / 2 \in \mathcal{B}(0, \delta)$ and then that $Z_{M_{0}}(u / 2)$ is well defined.

(1) Assume that $0<\gamma<1$. Then,

$$
\left|Z_{M_{0}}(u)\right|^{\gamma} \leq\left|Z_{M_{0}}\left(\frac{u}{2}\right)\right|^{\gamma}+\left|Z_{M_{0}}(u)-Z_{M_{0}}\left(\frac{u}{2}\right)\right|^{\gamma} .
$$

By Theorem 5.3, $Z_{M_{0}}$ has weakly stationary increments and $Z_{M_{0}}(0)=0$ almost surely. In particular,

$$
Z_{M_{0}}(u)-Z_{M_{0}}\left(\frac{u}{2}\right) \stackrel{(d)}{=} Z_{M_{0}}\left(\frac{u}{2}\right)
$$

Hence, since $\mathbb{P}\left(Z_{M_{0}}(u / 2) \neq 0\right)=1$,

$$
\mathbb{P}\left(Z_{M_{0}}\left(\frac{u}{2}\right) \neq 0, Z_{M_{0}}(u) \neq Z_{M_{0}}\left(\frac{u}{2}\right)\right)=\mathbb{P}\left(Z_{M_{0}}(u) \neq Z_{M_{0}}\left(\frac{u}{2}\right)\right)=1>0 .
$$

Therefore,

$$
\mathbb{E}\left|Z_{M_{0}}(u)\right|^{\gamma}<\mathbb{E}\left|Z_{M_{0}}\left(\frac{u}{2}\right)\right|^{\gamma}+\mathbb{E}\left|Z_{M_{0}}(u)-Z_{M_{0}}\left(\frac{u}{2}\right)\right|^{\gamma}
$$


since the inequality $(5.7)$ is strict on $\left\{Z_{M_{0}}(u) \neq 0, Z_{M_{0}}(u) \neq Z_{M_{0}}(u / 2)\right\}$. Furthermore, by (5.8),

$$
\mathbb{E}\left|Z_{M_{0}}(u)-Z_{M_{0}}\left(\frac{u}{2}\right)\right|^{\gamma}=\mathbb{E}\left|Z_{M_{0}}\left(\frac{u}{2}\right)\right|^{\gamma} .
$$

Therefore,

$$
\mathbb{E}\left|Z_{M_{0}}(u)\right|^{\gamma}<2 \mathbb{E}\left|Z_{M_{0}}\left(\frac{u}{2}\right)\right|^{\gamma} .
$$

Moreover, by Theorem 5.3, $Z_{M_{0}}$ satisfies the self-similarity property (5.4). This property applied with $\lambda=1 / 2$ leads to

$$
\mathbb{E}\left|Z_{M_{0}}(u)\right|^{\gamma}<2^{1-\gamma h\left(M_{0}\right)} \mathbb{E}\left|Z_{M_{0}}(u)\right|^{\gamma} .
$$

Then, since $\mathbb{E}\left|Z_{M_{0}}(u)\right|^{\gamma} \neq 0,1<2^{1-\gamma h\left(M_{0}\right)}$ which means that $h\left(M_{0}\right)<1 / \gamma$.

(2) Assume now that $\gamma \geq 1$. Furthermore, for any $0<\eta<1, \mathbb{E}\left|Z_{M_{0}}(u)\right|^{\eta}<+\infty$. The first part of this proof implies that $h\left(M_{0}\right)<1 / \eta$ for every $\eta \in(0,1)$. Also, taking the asymptotics as $\eta \rightarrow 1, h\left(M_{0}\right) \leq 1$.

\section{Proof of Corollary 5.5.}

(1) Assume that $Z_{M_{0}}$ is a centered Gaussian random field. By (5.4), since the Gaussian field $Z_{M_{0}}$ is non degenerate, for every $u \in \mathcal{B}(0, \delta)$,

$$
\mathbb{P}\left(Z_{M_{0}}(u) \neq 0\right)=1 .
$$

Moreover, for any $u \in \mathcal{B}(0, \delta)$,

$$
\mathbb{E} Z_{M_{0}}(u)^{2}<+\infty
$$

Then by Corollary $5.4, h\left(M_{0}\right) \leq 1$.

Let us consider $u \in \mathcal{B}(0, \delta) \backslash\{0\}$ and recall (see Proof of Theorem 5.3) that

$$
Z_{M_{0}}\left(v_{1}\right)-Z_{M_{0}}\left(v_{2}\right) \stackrel{(d)}{=}\|u\|^{-h\left(M_{0}\right)}\left\|v_{1}-v_{2}\right\|^{h\left(M_{0}\right)} Z_{M_{0}}(u)
$$

for every $v_{1}, v_{2} \in \mathcal{B}(0, \delta)$. As a consequence, for every $v_{1}, v_{2} \in \mathcal{B}(0, \delta)$,

$$
\operatorname{Var}\left(Z_{M_{0}}\left(v_{1}\right)-Z_{M_{0}}\left(v_{2}\right)\right)=C^{2}\left\|v_{1}-v_{2}\right\|^{2 h\left(M_{0}\right)}
$$

with $C=\|u\|^{-h\left(M_{0}\right)} \sqrt{\operatorname{Var}\left(\mathrm{Z}_{\mathrm{M}_{0}}(\mathrm{u})\right)}$. Since $Z_{M_{0}}(0)=0$ almost surely, we then have that

$$
\begin{aligned}
\operatorname{Cov}\left(Z_{M_{0}}(v), Z_{M_{0}}(w)\right) & =\frac{1}{2}\left[\operatorname{Var} Z_{M_{0}}(v)+\operatorname{Var} Z_{M_{0}}(w)-\operatorname{Var}\left(Z_{M_{0}}(v)-Z_{M_{0}}(w)\right)\right] \\
& =\frac{C^{2}}{2}\left(\|v\|^{2 h\left(M_{0}\right)}+\|w\|^{2 h\left(M_{0}\right)}-\|v-w\|^{2 h\left(M_{0}\right)}\right)
\end{aligned}
$$

for every $v, w \in \mathcal{B}(0, \delta)$.

This covariance is the covariance of an Euclidean fractional Brownian motion restricted to $\mathcal{B}(0, \delta)$. So that, in this case, if $Z_{M_{0}}$ is a Gaussian random field, it is an Euclidean fractional Brownian motion restricted to $\mathcal{B}(0, \delta)$.

(2) Assume that $Z_{M_{0}}$ is an $\alpha$-stable random field. Hence, by (5.4) and since the $\alpha$-stable random field $Z_{M_{0}}$ is non degenerate, for every $u \in \mathcal{B}(0, \delta)$,

$$
\mathbb{P}\left(Z_{M_{0}}(u) \neq 0\right)=1 \text {. }
$$


Hence, for any $\gamma<\alpha$ and any $u \in \mathcal{B}(0, \delta)$,

$$
\mathbb{E}\left|Z_{M_{0}}(u)\right|^{\gamma}<+\infty .
$$

Assertion 2 is then a consequence of Corollary 5.4.

\subsubsection{Examples}

We give in this section several examples, refering to [56] for the proofs.

5.2.4.1. Spherical moving-average fractional fields

Let $n \in \mathbb{N} \backslash\{0\}$ and $\mathbb{S}^{n}=\left\{x_{1}, x_{2}, \ldots, x_{n+1} \in \mathbb{R}, \sum_{i=1}^{n+1} x_{i}^{2}=1\right\}$ is the $n$-dimensional unit sphere. The distance $d$ on $\mathbb{S}^{n}$ is its geodesic distance. By convention, $\mathbb{S}^{0}=\{-1,1\}$.

In this part, we introduce some fields indexed by $\mathbb{S}^{n}$ owing a moving-average representation. Let us recall the representation for the Euclidean fractional $\alpha$-stable moving-average motion (see $[66,76]$ or the section devoted to representations) with index $H \in(0,1)$ and $\alpha \in(0,2]$

$$
B_{H, \alpha}(M)=\int_{\mathbb{R}^{n}}\left(\left\|M M^{\prime}\right\|^{H-n / \alpha}-\left\|\widetilde{O} M^{\prime}\right\|^{H-n / \alpha}\right) \mathrm{d} W_{\alpha}\left(M^{\prime}\right)
$$

where $\widetilde{O}$ is the origin of $\mathbb{R}^{n}$ and where $W_{\alpha}$ is a symmetric $\alpha$-stable random measure on $\mathbb{R}^{n}$ with Lebesgue measure as control measure. In the case $\alpha=2, W_{2}$ is a random Brownian measure and $B_{H, 2}$ is an Euclidean fractional Brownian motion.

In order to define spherical moving-average $\alpha$-stable fields, we replace $W_{\alpha}$ by a random measure on $\mathbb{S}^{n}$ and the Euclidean norm by the distance $d$ on $\mathbb{S}^{n}$. Let $\mathrm{d} x$ be the Lebesgue measure on $\mathbb{R}^{n}$. Then, in (5.9), the term $\left\|\widetilde{O} M^{\prime}\right\|^{H-n / \alpha}$ implies that the kernel $M^{\prime} \mapsto\left\|M M^{\prime}\right\|^{H-n / \alpha}-\left\|\widetilde{O} M^{\prime}\right\|^{H-n / \alpha}$ is in $L^{\alpha}\left(\mathbb{R}^{n}, \mathrm{~d} x\right)$ for any $H \in(0,1)$ so that $B_{H, \alpha}$ is well-defined. Without this correction term, the kernel will not be in $L^{\alpha}\left(\mathbb{R}^{n}, \mathrm{~d} x\right)$ in view of its behaviour as $\left\|M M^{\prime}\right\| \rightarrow+\infty$. Since the sphere is compact, we don't need to reproduce this term in our framework.

Let $\sigma_{n}$ be the uniform measure on $\mathbb{S}^{n}, \alpha \in(0,2]$. Let $W_{\alpha}$ be a symmetric $\alpha$-stable random measure on $\mathbb{S}^{n}$ with $\sigma_{n}$ as control measure when $0<\alpha<2$ and let $W_{2}$ be the Brownian random measure on $\mathbb{S}^{n}$ with $\sigma_{n}$ as control measure. Let us precise that $\int_{\mathbb{S}^{n}} f(M) \mathrm{d} W_{\alpha}(M)$ exists iff $f \in L^{\alpha}\left(\mathbb{S}^{n}, \sigma_{n}\right)$. Furthermore, if $f \in L^{\alpha}\left(\mathbb{S}^{n}, \sigma_{n}\right)$, then $\int_{\mathbb{S}^{n}} f(M) \mathrm{d} W_{\alpha}(M)$ is an $\alpha$-stable symmetric random variable and

$$
\forall u \in \mathbb{R}, \mathbb{E}\left[\exp \left(\mathrm{i} u \int_{\mathbb{S}^{n}} f(M) \mathrm{d} W_{\alpha}(M)\right)\right]=\exp \left[-|u|^{\alpha} \int_{\mathbb{S}^{n}}|f(M)|^{\alpha} \mathrm{d} \sigma_{n}(M)\right] .
$$

Let $H \in \mathbb{R}$ such that $H \neq n / \alpha$. As soon as

$$
X_{H, \alpha}(M)=\int_{\mathbb{S}^{n}} d\left(M, M^{\prime}\right)^{H-n / \alpha} \mathrm{d} W_{\alpha}\left(M^{\prime}\right), M \in \mathbb{S}^{n}
$$

is well-defined, with convention $0^{\beta}=+\infty$ for $\beta<0, X_{H, \alpha}$ is called spherical moving-average fractional $\alpha$-stable random field. Note that $X_{H, 2}$ is a Gaussian field.

Proposition 5.6. Let $H \in \mathbb{R}$ such that $H \neq n / \alpha$. Then, the spherical moving-average fractional $\alpha$-stable random field $X_{H, \alpha}$ is well-defined if and only if $H>0$.

This is a difference with the Euclidean case: this difference is due to the fact that the sphere is bounded.

Proposition 5.7. Let $H>0$ such that $H \neq n / \alpha$. 
(1) Then $X_{H, \alpha}$ has weakly stationary increments.

(2) (a) Assume $H \in(0,1)$. Then $X_{H, \alpha}$ is lass at each point with index $H$. Furthermore, the tangent field at point $M_{0}$ is an Euclidean moving-average $\alpha$-stable random field with index $H$. More precisely, for every $M_{0} \in \mathbb{S}^{n}$,

$$
\lim _{\varepsilon \rightarrow 0^{+}}\left(\frac{X_{H, \alpha}\left(M_{0}+\varepsilon v\right)-X\left(M_{0}\right)}{\varepsilon^{H}}\right)_{v \in B(0, \pi)} \stackrel{(d)}{=}\left(B_{H, \alpha}(v)\right)_{v \in B(0, \pi)},
$$

where $B_{H, \alpha}$ is defined by (5.9).

(b) Assume $H>1$. Then $X_{H, \alpha}$ is lass at each point with index 1. More precisely, for every $M_{0} \in \mathbb{S}^{n}$,

$$
\lim _{\varepsilon \rightarrow 0^{+}}\left(\frac{X_{H, \alpha}\left(M_{0}+\varepsilon v\right)-X\left(M_{0}\right)}{\varepsilon}\right)_{v \in B(0, \pi)} \stackrel{(d)}{=}\left(Z_{M_{0}, \alpha}(v)\right)_{v \in B(0, \pi)},
$$

where for every $v \in \mathcal{B}(0, \pi)$,

$$
Z_{M_{0}, \alpha}(v)=\left(\frac{n}{\alpha}-H\right) \int_{\mathbb{S}^{n}} \frac{\left\langle v, \Pi_{M_{0}}\left(M^{\prime}\right)\right\rangle}{\left\|\Pi_{M_{0}}\left(M^{\prime}\right)\right\|} d\left(M_{0}, M^{\prime}\right)^{H-1-n / \alpha} \mathrm{d} W_{\alpha}\left(M^{\prime}\right),
$$

with $\Pi_{M_{0}}$ the inverse of the exponential map $\exp _{M_{0}}$ at point $M_{0}$.

5.2.4.2. Spherical moving-average multifractional stable fields The previous examples have weakly stationary increments on $\mathbb{S}^{n}$, which implies that the index of the lass property at point $M_{0}$ does not depend on $M_{0}$. However, for modelization purpose, it is sometime a constraining condition. We therefore introduce some multifractional lass random fields: the index of the lass property will then vary. In the case of Euclidean random fields, multifractional random fields have been defined by replacing the index $H$ by a function $h(\cdot)$ in some integral representations of fractional Euclidean fields, e.g. $[2,8,9,11,63,69,79]$. The most famous examples are multifractional Brownian fields, introduced either by replacing the Hurst index $H$ by a function in the moving-average representation of a fractional Brownian motion [69] and in its harmonizable representation [8]. Following this approach, we define spherical moving-average multifractional $\alpha$-stable and Gaussian fields.

Let us recall that $\alpha \in(0,2]$ and consider $h: \mathbb{S}^{n} \longrightarrow(0,+\infty)$ such that $h(M) \neq n / \alpha$ for every $M \in \mathbb{S}^{n}$. Then,

$$
X_{h, \alpha}(M)=\int_{\mathbb{S}_{n}} d\left(M, M^{\prime}\right)^{h(M)-n / \alpha} \mathrm{d} W_{\alpha}\left(M^{\prime}\right), M \in \mathbb{S}^{n}
$$

is well-defined and $X_{h, \alpha}$ is called spherical moving-average multifractional $\alpha$-stable random field with multifractional function $h$. If $\alpha=2, X_{h, 2}$ is a centered Gaussian random field.

Proposition 5.8. Let $M_{0} \in \mathbb{S}^{n}$. Assume that the function $h$ is $\mathcal{C}^{1}$ and that $h\left(M_{0}\right)<1$. Then, $X_{h, \alpha}$ is lass at point $M_{0}$ with index $h\left(M_{0}\right)$ and its tangent field at point $M_{0}$ is an Euclidean moving-average $\alpha$-stable random field with index $h\left(M_{0}\right)$. More precisely,

$$
\lim _{\varepsilon \rightarrow 0^{+}}\left(\frac{X_{h, \alpha}\left(M_{0}+\varepsilon v\right)-X\left(M_{0}\right)}{\varepsilon^{h\left(M_{0}\right)}}\right)_{v \in B(0, \pi)} \stackrel{(d)}{=}\left(B_{h\left(M_{0}\right), \alpha}(v)\right)_{v \in B(0, \pi)},
$$

where $B_{h\left(M_{0}\right), \alpha}$ is defined by $(5.9)$.

\section{Estimation}

\subsection{Introduction}

There is a huge litterature, theoretical or applied, devoted to the estimation of the fractional index of random processes and fields. One bibliographical study has been done in 2001 [24], and since, numerous papers have 
been published. We don't claim that the following bibliography of this hot topics is exhaustive. Generalized quadratic variations have been introduced and studied in $[57,58]$. Several papers extend, and refine their uses $[5,14-17,25,27,71]$. Cramer-Rao bound and minimax rates for estimation of fractional index are studied in [28, $35,64]$. The estimation of multifractional functions began with $[9,11]$ and continues with, e.g., $[3,4,6,26]$. Study of anisotropic Gaussian fields has been done by $[18,33,55]$. Extension to non-Gaussian fields are studied in $[12,13,63]$. Extension to the sphere has been done by [54]. To sum up, the Gaussian and Euclidean case is well understood. The Gaussian and manifold cases have not been really studied, except the sphere's case [54]. The non-Gaussian case is not well understood.

The aim of this section is only to give the basic ideas in dimension one and in the unifractional case. The same ideas are indeed used in the other cases and we refer to the bibliography for further developments.

\subsection{An universal estimator of the local self-similarity index}

As presented in the section 5, the local self-similarity is a seminal concept. Estimating the lass index is therefore a very natural question. Following [30], we present here an estimator of the lass index. This estimator is rather universal, but unfortunatly has a very poor rate of convergence. Let us be more precise.

Let $X(t)$ be a stochastic process defined on $[a, b] \subset \mathbb{R}$. Let $t_{0} \in(a, b)$. For an index $H$, define the normalized increments of $X$

$$
Z\left(t, t^{\prime}\right)=\frac{X(t)-X\left(t^{\prime}\right)}{\left|t-t^{\prime}\right|^{H}}, \quad t \neq t^{\prime}
$$

The definition of the lass index is given by the following assumption. Assume that there exists a random variable $Y$ such that

$$
\lim _{t, t^{\prime} \rightarrow t_{0}} Z\left(t, t^{\prime}\right) \stackrel{(d)}{=} Y
$$

and that the family $\log ^{2}\left|Z\left(t, t^{\prime}\right)\right|$ is uniformly integrable in a neighbourhood of $t_{0}$.

For $\epsilon_{N}>2^{-N}$, define $\mathcal{V}_{N}$

$$
\mathcal{V}_{N}=\left\{k \in \mathbb{Z} \text { s.t. }\left|\frac{k}{2^{N}}-t_{0}\right| \leq \varepsilon_{N}\right\} .
$$

Let $v_{N}$ be the cardinal of the set $\mathcal{V}_{N}$

$$
v_{N}=\# \mathcal{V}_{N}
$$

Define the log-variation of the process in the neighborhood $\mathcal{V}_{N}$ of $t_{0}$

$$
\begin{aligned}
W_{N} & =\frac{-1}{N v_{N}} \sum_{k \in \mathcal{V}_{N}} \log _{2}\left|\Delta_{k, N} X\right|, \\
\Delta_{k, N} X & =X\left(\frac{k+1}{2^{N}}\right)-X\left(\frac{k}{2^{N}}\right), \\
\log _{2}(x) & =\log (x) / \log (2) .
\end{aligned}
$$

Theorem 6.1. Assume that $\varepsilon_{N} \rightarrow 0$ as $N \rightarrow+\infty$, with $\varepsilon_{N}>2^{-N}$. Then one has

$$
\lim _{N \rightarrow+\infty} W_{N} \stackrel{(\text { a.s. })}{=} H \text {. }
$$




\section{Comments on Theorem 6.1.}

We have to point out that, in Theorem 6.1, we don't need the assumption $v_{N} \rightarrow+\infty$. This is because the proofs don't rely on ergodic arguments. Indeed, this result is due to the behaviour of $\log |X(t)|$. Let us give an example. Let $B_{H}(t)$ be a fractional Brownian Motion of index $0<H<1$. Clearly, the variance of $\log \left|B_{H}(t)\right|$ does not depend on $t$. It follows that

$$
\lim _{t \rightarrow 0} \frac{\log \left|B_{H}(t)\right|}{\log |t|} \stackrel{\mathcal{L}^{2}}{=} H
$$

Proofs of Theorem 6.1. Let

$$
V_{N}=\frac{1}{v_{N}} \sum_{k \in \mathcal{V}_{N}} \log _{2}\left|2^{N H} \Delta_{k, N} X\right| .
$$

One has

$$
\mathbf{E} V_{N}=\frac{1}{v_{N}} \sum_{k \in \mathcal{V}_{N}} \mathbf{E} \log _{2}\left|2^{N H} \Delta_{k, N} X\right| .
$$

The sequence $\log ^{2}\left|Z\left(t, t^{\prime}\right)\right|$ is uniformly integrable, therefore the sequence $\log \left|Z\left(t, t^{\prime}\right)\right|$ is uniformly integrable too. It follows that

$$
\lim _{N \rightarrow+\infty} \mathbf{E} \log _{2}\left|2^{N H} \Delta_{k, N} X\right|=\mathbf{E} \log _{2}|Y|,
$$

and

$$
\lim _{N \rightarrow+\infty} \mathbf{E} V_{N}=\mathbf{E} \log _{2}|Y|
$$

The variance of $V_{N}$ is given by

$$
\begin{aligned}
\operatorname{var} V_{N}= & \frac{1}{v_{N}^{2}} \sum_{k, k^{\prime} \in \mathcal{V}_{N}} \mathbf{E}\left[\left\{\log _{2}\left|2^{N H} \Delta_{k, N} X\right|-\mathbf{E} \log _{2}\left|2^{N H} \Delta_{k, N} X\right|\right\}\right. \\
& \left.\times\left\{\log _{2}\left|2^{N H} \Delta_{k^{\prime}, N} X\right|-\mathbf{E} \log _{2}\left|2^{N H} \Delta_{k^{\prime}, N} X\right|\right\}\right] .
\end{aligned}
$$

By Cauchy-Schwarz inequality

$$
\operatorname{var} V_{N} \leq \frac{1}{v_{N}^{2}}\left\{\sum_{k \in \mathcal{V}_{N}} \sqrt{\operatorname{var} \log _{2}\left|2^{N H} \Delta_{k, N} X\right|}\right\}^{2} .
$$

Uniform integrability of $\log _{2}^{2}\left|2^{N H} \Delta_{k, N} X\right|$ yields

$$
\lim _{N \rightarrow+\infty} \operatorname{var} \log _{2}\left|2^{N H} \Delta_{k, N} X\right|=\operatorname{var} \log _{2}|Y| .
$$

The sequence var $V_{N}$ is therefore bounded

$$
\sup _{N \geq 1} \operatorname{var} V_{N}<\infty .
$$


Borel-Cantelli's lemma then implies

$$
\lim _{N \rightarrow+\infty} \frac{V_{N}}{N} \stackrel{(a . s .)}{=} 0 .
$$

Basic computations leads to

$$
\frac{V_{N}}{N}=H-W_{N},
$$

and Theorem 6.1 is proved.

Let us now consider the rates of convergence.

Let $X$ be a process satisfying conditions of Theorem 6.1. Let $c>0, c \neq 1$. Process $c X$ satisfies of course conditions of Theorem 6.1. Denote by $W_{N}(X)$ (resp. $\left.W_{N}(c X)\right)$ the log-variations of $X$ (resp. $\left.c X\right)$ defined in $(6.1)$ associated with the discretization step $2^{-N}$.

It follows from (6.2) and (6.3) that

$$
\sup _{N \geq 1} \mathbf{E} V_{N}^{2}<\infty
$$

and

$$
\mathbf{E}\left(W_{N}(X)-H\right)^{2} \leq \frac{\sup _{N \geq 1} \mathbf{E} V_{N}^{2}}{N^{2}} .
$$

Suppose that there exists a process $X$ for which the rate of convergence is better, hence

$$
\lim _{N \rightarrow+\infty} N^{2} \mathbf{E}\left(W_{N}(X)-H\right)^{2}=0 .
$$

By Cauchy-Schwarz

$$
\lim _{N \rightarrow+\infty} N \mathbf{E}\left|W_{N}(X)-H\right|=0
$$

Basic computations lead to

$$
W_{N}(c X)=W_{N}(X)-\frac{\log _{2} c}{N}
$$

so that

$$
\mathbf{E}\left(W_{N}(c X)-H\right)^{2}=\frac{\log _{2}^{2} c}{N^{2}}-\frac{2 \log _{2} c}{N} \mathbf{E}\left(W_{N}(X)-H\right)+\mathbf{E}\left(W_{N}(X)-H\right)^{2},
$$

and

$$
\lim _{N \rightarrow+\infty} N^{2} \mathbf{E}\left(W_{N}(c X)-H\right)^{2}=\log _{2}^{2} c
$$

The mean squared error is only the square of the logarithm of the mesh $2^{-N}$ of the grid. 


\subsection{Long range dependance and increments of the $\mathrm{fBm}$}

Let $Z_{N}, N \geq 0$ be a stationary second-order process. Sequence $Z$ is said to exhibit a long-range dependence (e.g. [75]) if the series $\sum \operatorname{cov}\left(Z_{0}, Z_{N}\right)$ diverges and a short range dependence if this series converges. Let $X(t), t \in \mathbb{R}$ be an Euclidean fractional Brownian motion. Let

$$
\Delta_{p} X=N^{2 H}\left(X\left(\frac{p+1}{N}\right)-X\left(\frac{p}{N}\right)\right)
$$

be the normalized first order increment of $X$ with mesh $1 / N$. Does these increments exhibits a long range dependence? When $H=1 / 2$, the answer is clearly negative and we only consider the case $H \neq 1 / 2$. A Taylor expansion of order 2 shows that the series $\sum \operatorname{cov}\left(\Delta_{0} X, \Delta_{p} X\right)$ is convergent for $H<1 / 2$ and divergent for $H>1 / 2$. One usually says that the increments of the fractional Brownian motion are persistent when $H>1 / 2$. Let now consider the normalized second order increments

$$
\Gamma_{p} X=N^{2 H}\left(X\left(\frac{p+1}{N}\right)-2 X\left(\frac{p}{N}\right)+X\left(\frac{p-1}{N}\right)\right) .
$$

A Taylor expansion of order 3 shows that the series $\sum \operatorname{cov}\left(\Gamma_{0} X, \Gamma_{p} X\right)$ is convergent. There is no long range dependence for the second order increments. This raises deep questions in terms of modeling long range phenomena, but that is another story! We will see in the next section that the square of the first order increments exhibit long range dependance if $H>3 / 4$ and short range dependance for $H<3 / 4$. But the square of the second order increments exhibits always a short range dependence. This property will be intensively used in the sequel.

\subsection{Generalized quadratic variations}

\subsubsection{Quadratic variations}

Let $X$ be a centered stationary normalized Gaussian process on $[0,1]$ with the covariance function $r(t)=$ $\mathbb{E} X(0) X(t)$. Let us assume the expansion

$$
r(t)=1-|t|^{2 H} L(t)
$$

where $L(t)$ is a slowly varying function in zero, that is, a function $L$ such that, for all $a>0$,

$$
\lim _{x \rightarrow+\infty} f(a x) / f(x)=1 .
$$

Quadratic variations on $[0,1]$ of process $X$ at scale $1 / N$ are defined by

$$
V_{N}=\sum_{k=0}^{N-1}\left(X\left(\frac{k+1}{N}\right)-X\left(\frac{k}{N}\right)\right)^{2} .
$$

A classical result (e.g. [47]) ensures that

$$
\lim _{N \rightarrow+\infty} N^{2 H-1} V_{N} \stackrel{\text { (a.s.) }}{=} 1 \text {. }
$$

The quadratic variations can therefore be used to identify parameter $H$

$$
\widehat{H}_{N}=\frac{1}{2}+\frac{1}{2} \log _{2} \frac{V_{N / 2}}{V_{N}}
$$


When considering the limiting distribution of the quadratic variations, Guyon and Leon [47] found two cases

(1) $0<H<3 / 4$. The variable $\sqrt{N}\left(N^{2 H-1} V_{n}-1\right)$ converges in distribution, as $N \rightarrow+\infty$, to a Gaussian variable;

(2) $3 / 4<H<1$. The variable $N^{2-2 H}\left(N^{2 H-1} V_{n}-1\right)$ converges in distribution, as $N \rightarrow+\infty$, to a nonGaussian variable.

Therefore, the rate of convergence of estimator of $H$ based on quadratic variations dramatically decreases when $3 / 4<H<1$.

\subsubsection{Extension of quadratic variations}

Although non-differentiable processes are considered, $[57,58]$ introduce generalized quadratic variations associated with discrete second order derivative. Let $a_{k}, k=0, \ldots, K$ be a discrete sequence of reals satisfying

$$
\begin{gathered}
\sum_{k=0}^{K} a_{k}=0, \\
\sum_{k=0}^{K} k a_{k}=0, \\
\sum_{k=0}^{K} k^{2} a_{k} \neq 0 .
\end{gathered}
$$

Sequence $\left(a_{k}\right)$ acts like a discrete second order derivative. For instance, sequence $-1,1$ does not fulfill these conditions, but sequence $1,-2,1$ does. Generalized quadratic variations of process $X$ on $[0,1]$ at scale $1 / N$ associated with sequence $\left(a_{k}\right)$ are defined by

$$
V_{N}=\sum_{p=0}^{N-K}\left(\Delta_{p} X\right)^{2},
$$

where

$$
\Delta_{p} X=\sum_{k=0}^{K} a_{k} X\left(\frac{k+p}{N}\right) .
$$

We give the case corresponding to Filtered White Noises [10]:

\section{A.6.2.}

Filtered White Noises.

Let $g(t, \lambda)$ be the following harmonizable fractional-integral type kernel

$$
g(t, \lambda)=\frac{a(t)}{|\lambda|^{\frac{1}{2}+H}}+\varepsilon(t, \lambda)
$$

with $a(t) \in C^{2}, \varepsilon(t, \lambda) \in C^{2,2}$ satisfying, for $i, j=0,1,2$

$$
\left|\frac{\partial^{i+j}}{\partial t^{i} \partial \lambda^{j}} \varepsilon(t, \lambda)\right| \leq \frac{C}{|\lambda|^{\frac{1}{2}+\eta+j}},
$$

with $\eta>H$. $C$ denotes a generic constant that can change from an occurrence to another. 
Let $W$ be a standard Brownian measure. Define process $X$ by

$$
X(t)=\int_{\mathbb{R}} g(t, \lambda)\left(\mathrm{e}^{\mathrm{i} t \lambda}-1\right) \mathrm{d} W(\lambda) .
$$

Process $X$ is called a Filtered White Noise. When $a(t)=1$ and $\varepsilon(t, \lambda)=0$, the resulting process is a fractional Brownian motion. By construction, the value of a Filtered White Noise at 0 is equal to zero. This restriction does not matter: the identification results we present below are still valid if we add an arbitrary random variable to a Filtered White Noise. The function $\varepsilon$ is clearly a remainder term, and conditions on this function are given to ensure that $\varepsilon$ really is a remainder term. Even if it is difficult to explain without a deep inspection of the proof, the condition $a \in C^{2}$ implies a decrease of the increments of orders 1 and 2 of the process.

We should wonder whether the Gaussian stationary processes considered for instance by [57-59], and that satisfy more or less a condition like (6.4), can be viewed as Filtered White Noise. Such is the case, via a minor modification of the process. Let $f(\lambda)$ be the spectral density of a Gaussian centered stationary process $Y$. By Bochner's theorem, this spectral density is a positive function. Process $Y$ can then be represented through a stochastic integral with respect to a Brownian measure $W$

$$
Y(t)=\int_{\mathbb{R}} \sqrt{f(\lambda)} \mathrm{e}^{\mathrm{i} t \lambda} \mathrm{d} W(\lambda) .
$$

Process $X(t)=Y(t)-Y(0)$ is then represented as follows

$$
X(t)=\int_{\mathbb{R}} \sqrt{f(\lambda)}\left(\mathrm{e}^{\mathrm{i} t \lambda}-1\right) \mathrm{d} W(\lambda) .
$$

The estimator of parameter $H$ derived from the generalized quadratic variations is then as follows

$$
\widehat{H}_{N}=\frac{1}{2}+\frac{1}{2} \log _{2} \frac{V_{N / 2}}{V_{N}}
$$

Theorem 6.3. Let $X$ be a process satisfying A.6.2.

(1) Strong consistency.

$$
\lim _{N \rightarrow+\infty} \widehat{H}_{N} \stackrel{(a . s .)}{=} H
$$

(2) Asymptotic normality.

As $N \rightarrow+\infty, \sqrt{N}\left(\widehat{H}_{N}-H\right)$ converges in distribution to a centered Gaussian variable.

Remark 6.4. Non-centered processes.

Let $X$ be a process satisfying A.6.2. Let $m(t)$ be $C^{2}$. Then Theorem 6.3 holds for process $Y(t)=X(t)+m(t)$.

To prove this remark, it is sufficient to notice that

$$
V_{N}=\sum_{p=0}^{N-K}\left(\Delta_{p} Y\right)^{2}+\sum_{p=0}^{N-K}\left(\Delta_{p} m\right)^{2}+2 \sum_{p=0}^{N-K}\left(\Delta_{p} Y \Delta_{p} m\right)
$$

where $V_{N}$ are the variations associated with process $Y$. 
Proof of Theorem 6.3. The study of the $V_{N}$ requires estimates of their expectations and variances. Note that

$$
\begin{aligned}
\mathbb{E} V_{N} & =\sum_{p=0}^{N-K} \mathbb{E}\left(\sum_{k=0}^{K} a_{k} X\left(\frac{k+p}{N}\right)\right)^{2} \\
& =\sum_{p=0}^{N-K} \int_{\mathbb{R}} \sum_{k=0}^{K}\left|a_{k} g\left(\frac{k+p}{N}, \lambda\right) e\left(\lambda \frac{k+p}{N}\right)\right|^{2} \mathrm{~d} \lambda,
\end{aligned}
$$

where function $e$ stands for $e(\lambda)=\mathrm{e}^{\mathrm{i} \lambda}-1$.

Using the expansion for $g(t, \lambda)$, we can express $\mathbb{E} V_{N}$ as a sum of terms, each of them being of the following form $(p=0, \ldots, N-K)$

$$
\begin{aligned}
I\left(S, S^{\prime}\right)_{p, p^{\prime}}= & \int_{\mathbb{R}} \sum_{k, k^{\prime}=0}^{K} a_{k} a_{k^{\prime}} S\left(\frac{k+p}{N}, N u\right) S^{\prime}\left(\frac{k^{\prime}+p^{\prime}}{N}, N u\right) \\
& \left.\times e((k+p) u) \bar{e}\left(\left(k^{\prime}+p^{\prime}\right) u\right)\right) N \mathrm{~d} u .
\end{aligned}
$$

A change of variables $u=N \lambda$ has been performed and each $S, S^{\prime}$ stands for one of the functions at the right hand-side of (6.5), so that either $S(t, \lambda)=\frac{a(t)}{|\lambda|^{H+1 / 2}}$ or $S(t, \lambda)$ is bounded by such a term, and the same holds for $S^{\prime}$.

The following condition is implied by A.6.2 if $S$ stands for one of the functions at the right hand-side of (6.5). $S(t, \lambda) \in C^{2,2}\left([0,1] \times \mathbb{R}^{*}\right)$ and

$$
\left|\frac{\partial^{i+j}}{\partial^{i} t \partial^{j} \lambda} S(t, \lambda)\right| \leq \frac{C}{|\lambda|^{\frac{1}{2}+\delta+j}},
$$

for $i=0$ to 2 and $j=0$ to 2 with $0<\delta<1$, and the same holds for $S^{\prime}$.

Since $X$ is a Gaussian process, the variance of $V_{N}$ is given by

$$
\begin{aligned}
\operatorname{var}\left(V_{N}\right)= & 2 \sum_{p, p^{\prime}=0}^{N-K}\left(\int_{\mathbb{R}} \sum_{k, k^{\prime}=0}^{K} a_{k} a_{k^{\prime}} g\left(\frac{k+p}{N}, \lambda\right) g\left(\frac{k^{\prime}+p^{\prime}}{N}, \lambda\right)\right. \\
& \left.\times e\left(\lambda \frac{k+p}{N}\right) \bar{e}\left(\lambda \frac{k^{\prime}+p^{\prime}}{N}\right) \mathrm{d} \lambda\right)^{2}
\end{aligned}
$$

which is a sum of terms of the form $I\left(S, S^{\prime}\right)_{p, p^{\prime}}$. The estimation of the expectation and variance of $V_{N}$ first requires the estimation of the $I\left(S, S^{\prime}\right)_{p, p^{\prime}}$. We therefore state the following result on the $I\left(S, S^{\prime}\right)_{p, p^{\prime}}$ now.

Lemma 6.5. The following bound holds for $N$ large enough.

$$
\left|I\left(S, S^{\prime}\right)_{p, p^{\prime}}\right| \leq \frac{C}{N^{\delta+\delta^{\prime}}\left(1+\left(p-p^{\prime}\right)^{2}\right)} .
$$

Proof of Lemma 6.5. We use a Taylor expansion of $S$ of order 2 for $0 \leq k \leq K$

$$
S\left(\frac{k+p}{N}, N u\right)=\sum_{j=0}^{2} \frac{\partial^{j}}{\partial t^{j}} S\left(\frac{p}{N}, N u\right) \frac{k^{j}}{N^{j} j !}+\frac{k^{2}}{2 N^{2}} \frac{\partial^{2}}{\partial t^{2}} S\left(\frac{\kappa+p}{N}, N u\right),
$$


where $0 \leq \kappa \leq K$. The same holds for $S^{\prime}$. We then obtain the following expansion for $I\left(S, S^{\prime}\right)_{p, p^{\prime}}$

$$
\begin{aligned}
I\left(S, S^{\prime}\right)_{p, p^{\prime}}= & \sum_{j=0}^{2} N^{-j} \sum_{j_{1}+j_{2}=j} \frac{1}{j_{1} ! j_{2} !} \int_{\mathbb{R}}\left[\sum_{k, k^{\prime}=0}^{K} a_{k} a_{k^{\prime}} k^{j_{1}} k^{\prime j_{2}} e((k+p) u) \bar{e}\left(\left(k^{\prime}+p^{\prime}\right) u\right)\right] \\
& \times \frac{\partial^{j_{1}}}{\partial t^{j_{1}}} S\left(\frac{p}{N}, N u\right) \frac{\partial^{j_{2}}}{\partial t^{j_{2}}} S^{\prime}\left(\frac{p^{\prime}}{N}, N u\right) N \mathrm{~d} u \\
& +\sum_{j=2}^{4} N^{-j} \sum_{j_{1}+j_{2}=j} \frac{1}{j_{1} ! j_{2} !} \int_{\mathbb{R}} \sum_{k, k^{\prime}=0}^{K} a_{k} a_{k^{\prime}} k^{j_{1}} k^{j_{2}} e((k+p) u) \bar{e}\left(\left(k^{\prime}+p^{\prime}\right) u\right) \\
& \times \frac{\partial^{j_{1}}}{\partial t^{j_{1}}} S\left(\frac{\epsilon\left(j_{1}\right) \kappa+p}{N}, N u\right) \frac{\partial^{j_{2}}}{\partial t^{j_{2}}} S^{\prime}\left(\frac{\epsilon\left(j_{2}\right) \kappa^{\prime}+p^{\prime}}{N}, N u\right) N \mathrm{~d} u,
\end{aligned}
$$

where $\epsilon(j)=1$ if $j=2,0$ otherwise.

Let us first consider the case $p=p^{\prime}$. We have to bound $\left|I\left(S, S^{\prime}\right)_{p, p}\right|$ by $C N^{-\delta-\delta^{\prime}}$.

We clearly have

$$
\sum_{k, k^{\prime}=0}^{K} a_{k} a_{k^{\prime}} k^{j_{1}} k^{\prime j_{2}} e((k+p) u) \bar{e}\left(\left(k^{\prime}+p\right) u\right)=\sum_{k, k^{\prime}=0}^{K} a_{k} a_{k^{\prime}} k^{j_{1}} k^{\prime j_{2}} \mathrm{e}^{\mathrm{i} u\left(k-k^{\prime}\right)} .
$$

Each integral of (6.10) is bounded by a term involving

$$
\int_{\mathbb{R}}\left|\sum_{k, k^{\prime}=0}^{K} a_{k} a_{k^{\prime}} k^{j_{1}} k^{\prime j_{2}} \mathrm{e}^{\mathrm{i} u\left(k-k^{\prime}\right)}\right| \frac{\mathrm{d} u}{|u|^{\delta+\delta^{\prime}+1}} .
$$

The function $\sum_{k, k^{\prime}=0}^{K} a_{k} a_{k^{\prime}} k^{j_{1}} k^{\prime j_{2}} \mathrm{e}^{\mathrm{i} u\left(k-k^{\prime}\right)}$ and its derivatives up to order 2 vanish at $u=0$ hence $\sum_{k, k^{\prime}=0}^{K} a_{k} a_{k^{\prime}} k^{j_{1}} k^{\prime j_{2}} \mathrm{e}^{\mathrm{i} u\left(k-k^{\prime}\right)}=o\left(|u|^{2(n+1)}\right)$ when $|u| \rightarrow 0$. Then

$$
\int_{0^{+}}^{1}\left|\sum_{k, k^{\prime}=0}^{K} a_{k} a_{k^{\prime}} k^{j_{1}} k^{\prime j_{2}} \mathrm{e}^{\mathrm{i} u\left(k-k^{\prime}\right)}\right| \frac{\mathrm{d} u}{|u|^{\delta+\delta^{\prime}+1}}<+\infty
$$

since $\delta+\delta^{\prime}<2$. Moreover, since $\delta+\delta^{\prime}>0$ the integral (6.12) is convergent at infinity and therefore each term of line (6.10) is an $O\left(N^{-j-\delta-\delta^{\prime}}\right)$. It remains to bound (6.11). Each integral of (6.11) is bounded by

$$
C N^{-\delta-\delta^{\prime}}\left|\sum_{k, k^{\prime}=0}^{K} a_{k} a_{k^{\prime}} k^{j_{1}} k^{\prime j_{2}}\right| \int_{\mathbb{R}}\left|e((k+p) u) e\left(\left(k^{\prime}+p\right) u\right)\right| \frac{\mathrm{d} u}{|u|^{\delta+\delta^{\prime}+1}} .
$$

As $|u| \rightarrow \infty, e((k+p) u) \leq O(1)$ hence

$$
\int_{1}^{+\infty}\left|e((k+p) u) e\left(\left(k^{\prime}+p^{\prime}\right) u\right)\right| \frac{\mathrm{d} u}{|u|^{\delta+\delta^{\prime}+1}} \leq O(1),
$$

and as $|u| \rightarrow 0, e((k+p) u)=O((k+p) u$ hence

$$
\int_{0^{+}}^{1}\left|e((k+p) u) e\left(\left(k^{\prime}+p^{\prime}\right) u\right)\right| \frac{\mathrm{d} u}{|u|^{\delta+\delta^{\prime}+1}} \leq o\left(p p^{\prime}\right) .
$$


Since $p<N,(6.11)$ is bounded by $O\left(N^{-\delta-\delta^{\prime}}\right)$. Lemma 6.5 is proved for $p=p^{\prime}$.

It remains to prove Lemma 6.5 when $p \neq p^{\prime}$. Expression (6.10) leads to the integral factor

$$
\int_{\mathbb{R}} \mathrm{e}^{\mathrm{i} u\left(p-p^{\prime}\right)}\left[\sum_{k, k^{\prime}=0}^{K} a_{k} a_{k^{\prime}} k^{j_{1}} k^{\prime j_{2}} \mathrm{e}^{\mathrm{i} u\left(k-k^{\prime}\right)}\right] \frac{\partial^{j_{1}}}{\partial t^{j_{1}}} S\left(\frac{p}{N}, N u\right) \frac{\partial^{j_{2}}}{\partial t^{j_{2}}} S^{\prime}\left(\frac{p^{\prime}}{N}, N u\right) N \mathrm{~d} u .
$$

We integrate by parts twice and this gives

$$
\int_{\mathbb{R}} \frac{\mathrm{e}^{\mathrm{i} u\left(p-p^{\prime}\right)}}{\left(p-p^{\prime}\right)^{2}} \frac{\partial^{2}}{\partial u^{2}}\left[\left(\sum_{k, k^{\prime}=0}^{K} a_{k} a_{k^{\prime}} k^{j_{1}} k^{\prime j_{2}} \mathrm{e}^{\mathrm{i} u\left(k-k^{\prime}\right)}\right) \frac{\partial^{j_{1}}}{\partial t^{j_{1}}} S\left(\frac{p}{N}, N u\right) \frac{\partial^{j_{2}}}{\partial t^{j_{2}}} S^{\prime}\left(\frac{p^{\prime}}{N}, N u\right)\right] N \mathrm{~d} u .
$$

To prove that the previous integral converges, and that all terms coming from integrated terms in the integration by parts vanish, we only have to prove the absolute convergence of the terms given by the second derivative with respect to $u$ of

$$
\psi\left(u, j_{1}, j_{2}\right) \frac{\partial^{j_{1}}}{\partial t^{j_{1}}} S\left(\frac{p}{N}, N u\right) \frac{\partial^{j_{2}}}{\partial t^{j_{2}}} S^{\prime}\left(\frac{p^{\prime}}{N}, N u\right),
$$

where $\psi\left(u, j_{1}, j_{2}\right)=\sum_{k, k^{\prime}=0}^{K} a_{k} a_{k^{\prime}} k^{j_{1}} k^{\prime j_{2}} \mathrm{e}^{\mathrm{i} u\left(k-k^{\prime}\right)}$. Clearly, as $|u|$ goes to $\infty,\left|\frac{\partial^{i}}{\partial u^{i}} \psi\left(u, j_{1}, j_{2}\right)\right|=O(1)$ for $i=0,1,2$. This implies the convergence of (6.13) as $|u| \rightarrow \infty$. To have convergence when $|u| \rightarrow 0$ let us remark that $\left|\frac{\partial^{i}}{\partial u^{i}} \psi\left(u, j_{1}, j_{2}\right)\right|=o\left(|u|^{3-i}\right)$, when $|u| \rightarrow 0$. Then

$$
\left|\frac{\partial^{i_{1}} \psi\left(u, j_{1}, j_{2}\right)}{\partial u^{i_{1}}} \frac{\partial^{i_{2}+j_{1}}}{\partial t^{j_{1}} \partial u^{i_{2}}} S\left(\frac{p}{N}, N u\right) \frac{\partial^{i_{3}+j_{2}}}{\partial t^{j_{2}} \partial u^{i_{3}}} S^{\prime}\left(\frac{p^{\prime}}{N}, N u\right)\right| \leq \frac{C|u|^{3-\left(i_{1}+i_{2}+i_{3}\right)-\left(\delta+\delta^{\prime}+1\right)}}{N^{\delta+\delta^{\prime}+1+i_{2}+i_{3}}},
$$

for $i_{1}+i_{2}+i_{3}=2$. Hence each term of the first line $\left(c f\right.$. (6.10)) of the expansion of $I\left(S, S^{\prime}\right)_{p, p^{\prime}}$ is of order $\frac{1}{N^{\delta+\delta^{\prime}+j}\left(p-p^{\prime}\right)^{2}}$. We use a similar upper bound $O\left(N^{-\delta-\delta^{\prime}-2}\right)$ for the second line $(c f .(6.11))$ of the expansion of $I\left(S, S^{\prime}\right)_{p, p^{\prime}}$. Since $p, p^{\prime}<N$, we have proved Lemma 6.5 for $p \neq p^{\prime}$.

A second technical lemma relates the asymptotic behavior of $I(S, S)_{p, p^{\prime}}$ when $S(t, \lambda)=\frac{a(t)}{|\lambda|^{H+\frac{1}{2}}}$ to the function

$$
F_{\gamma}(x)=\int_{\mathbb{R}} \sum_{k, k^{\prime}=0}^{K} a_{k} a_{k^{\prime}} \frac{\mathrm{e}^{\mathrm{i}\left(x+k-k^{\prime}\right) u}}{|u|^{\gamma+1}} \mathrm{~d} u
$$

\section{Lemma 6.6.}

$$
I(S, S)_{p, p^{\prime}}=N^{-\delta-\delta^{\prime}} a(p \Delta) a\left(p^{\prime} \Delta\right) F_{2 H}\left(p-p^{\prime}\right)+O\left(\frac{1}{N^{\delta+\delta^{\prime}+1}\left(1+\left(p-p^{\prime}\right)^{2}\right)}\right) .
$$

Proof of Lemma 6.6. To begin with, we use the same Taylor expansion of $a$ at order 2 to get the expansions (6.10) and (6.11)

$$
\begin{aligned}
I_{p, p^{\prime}}= & \int_{\mathbb{R}}\left[\sum_{k, k^{\prime}=0}^{K} a_{k} a_{k^{\prime}} e((k+p) u) \bar{e}\left(\left(k^{\prime}+p^{\prime}\right) u\right)\right] \frac{a(p \Delta) a\left(p^{\prime} \Delta\right)}{N^{\delta+\delta^{\prime}}|u|^{\delta+\delta^{\prime}+1}} \mathrm{~d} u \\
& +O\left(\frac{1}{N^{\delta+\delta^{\prime}+1}\left(1+\left(p-p^{\prime}\right)^{2}\right)}\right) .
\end{aligned}
$$


Using

$$
\sum_{k, k^{\prime}=0}^{K} a_{k} a_{k^{\prime}} e((k+p) u) \bar{e}\left(\left(k^{\prime}+p^{\prime}\right) u\right)=\left(\sum_{k, k^{\prime}=0}^{K} a_{k} a_{k^{\prime}} \mathrm{e}^{\mathrm{i}\left(k-k^{\prime}\right) u}\right) \mathrm{e}^{\mathrm{i}\left(p-p^{\prime}\right) u},
$$

and Lemma 6.6 follows.

Now we describe the asymptotic behavior of the expectation and variance of the $V_{N}$ as $N$ goes to infinity.

Proposition 6.7. As $N \rightarrow \infty$ the following convergences hold

$$
\begin{aligned}
N^{2 H-1} \mathbb{E} V_{N} & \rightarrow F_{2 H}(0) \int_{0}^{1} a^{2}(t) \mathrm{d} t \\
N^{4 H-1} \operatorname{var}\left(V_{N}\right) & \rightarrow 2 \sum_{q=-\infty}^{\infty} F_{2 H}^{2}(q) \int_{0}^{1} a^{4}(t) \mathrm{d} t .
\end{aligned}
$$

Proof of Proposition 6.7. Let us recall that (6.7) expresses $\mathbb{E} V_{N}$ as a sum of terms that can be written as $I\left(S, S^{\prime}\right)_{p, p^{\prime}}$. Using expansion (6.5), each integral

$$
\int_{\mathbb{R}} \sum_{k, k^{\prime}=0}^{K} a_{k} a_{k^{\prime}} g\left(\frac{k+p}{N}, \lambda\right) g\left(\frac{k^{\prime}+p}{N}, \lambda\right) e\left(\lambda \frac{k+p}{N}\right) \bar{e}\left(\lambda \frac{k^{\prime}+p}{N} \lambda\right) \mathrm{d} \lambda,
$$

can be written

$$
\begin{aligned}
I= & I_{p, p}\left(\frac{a(t)}{|\lambda|^{H+1 / 2}}, \frac{a(t)}{|\lambda|^{H+1 / 2}}\right) \\
& +2 I_{p, p}\left(\frac{a(t)}{|\lambda|^{H+1 / 2}}, \varepsilon(t, \lambda)\right)+I_{p, p}(\varepsilon(t, \lambda), \varepsilon(t, \lambda)),
\end{aligned}
$$

where a change of variables $u=\frac{\lambda}{N}$ has been performed.

Clearly, $I_{p, p}\left(\frac{a(t)}{|\lambda|^{H+1 / 2}}, \frac{a(t)}{|\lambda|^{H+1 / 2}}\right)$ is the preponderant term as $N \rightarrow \infty$. Applying Lemma 6.6 to this term and Lemma 6.5 to the others, we get

$$
\mathbb{E} V_{N}=\sum_{p=0}^{N-K}\left[N^{-2 H} a^{2}\left(\frac{p}{N}\right) F_{2 H}(0)+O\left(N^{-2 H-1}\right)\right] .
$$

By standard results on Riemann's sums

$$
\frac{1}{N} \sum_{p=0}^{N-K} a^{2}\left(\frac{p}{N}\right)=\int_{0}^{1} a^{2}(t) \mathrm{d} t+O\left(\frac{1}{N}\right) .
$$

And the first part of Proposition 6.7 follows. 
Let us now prove the asymptotic behavior of the variance of the $V_{N}$, which is quite similar to the previous proof. Expansion (6.5), Lemmas 6.5 and 6.6 imply

$$
\begin{aligned}
\operatorname{var}\left(V_{N}\right)= & 2 \sum_{p, p^{\prime}=0}^{N-K}\left\{N^{-2 H} a\left(\frac{p}{N}\right) a\left(\frac{p^{\prime}}{N}\right) F_{2 H}\left(p-p^{\prime}\right)\right. \\
& \left.+O\left(\frac{1}{N^{2 H+1}}\right)\left(1+\left(p-p^{\prime}\right)^{2}\right)\right\}^{2} .
\end{aligned}
$$

We focus on the main term $2 N^{-4 H} \sum_{p, p^{\prime}=0}^{N-K} a^{2}\left(\frac{p}{N}\right) a^{2}\left(\frac{p^{\prime}}{N}\right) F_{2 H}^{2}\left(p-p^{\prime}\right)$. Set $q=p-p^{\prime}$ and $q^{\prime}=p+p^{\prime}$. The sum is then splitted for $|q|<Q$ and $|q| \geq Q$, where $Q$ is prescribed later. The second part is then

$$
N^{-4 H} \sum_{q^{\prime}=0}^{2(N-K)} \sum_{|q| \geq Q} a^{2}\left(\frac{q+q^{\prime}}{2 N}\right) a^{2}\left(\frac{q-q^{\prime}}{2 N}\right) F_{2 H}^{2}(q) .
$$

Since $\left|F_{2 H}(q)\right| \leq \frac{C}{1+q^{2}}$, this sum can be bounded by $O\left(N^{-4 H} \sum_{q^{\prime}=0}^{2(N-K)} \sum_{|q| \geq Q} \frac{1}{\left(1+q^{2}\right)^{2}}\right)$. Fix $Q$ large enough, this sum is then less than $O\left(\frac{1}{N^{4 H-1} Q^{3}}\right)$. Consider now the second part

$$
T=N^{-4 H} \sum_{q^{\prime}=0}^{2(N-K)} \sum_{|q| \leq Q} a^{2}\left(\frac{q+q^{\prime}}{2 N}\right) a^{2}\left(\frac{q-q^{\prime}}{2 N}\right) F_{2 H}^{2}(q)
$$

Permuting the sums and using standard results on Riemann's sums,

$$
T=\frac{1}{N^{4 H-1}} \sum_{|q| \leq Q} F_{2 H}^{2}(q)\left(\int_{0}^{1} a^{2}\left(s+\frac{q}{2 N}\right) a^{2}\left(s-\frac{q}{2 N}\right) \mathrm{d} s+O\left(\frac{1}{N}\right)\right)
$$

By a Taylor's expansion of $a$, we get

$$
T=\frac{1}{N^{4 H-1}} \sum_{|q| \leq Q} F_{2 H}^{2}(q)\left(\int_{0}^{1} a^{4}(s) \mathrm{d} s+O\left(\frac{q}{N}\right)\right)
$$


Since $\left|F_{2 H}(q)\right| \leq \frac{C}{1+q^{2}}, T$ is asymptotically equivalent to $\frac{1}{N^{4-1}} \int_{0}^{1} a^{4}(s) \mathrm{d} s \sum_{q=-\infty}^{q=+\infty} F_{2 H}^{2}(q)$. Hence

$$
\operatorname{var}\left(V_{N}\right)=\frac{2}{N^{4 H-1}} \int_{0}^{1} a^{4}(s) \mathrm{d} s \sum_{q=-\infty}^{q=+\infty} F_{2 H}^{2}(q)+O\left(\frac{1}{N^{3 H+\eta-1}}\right)
$$

and Proposition 6.7 is proved.

We can now prove the almost sure convergence and central limit theorem for generalized quadratic variations.

Proposition 6.8. The following limit holds

$$
\lim _{N \rightarrow \infty} \frac{V_{N}}{\mathbb{E} V_{N}}=1 \quad \text { a.s. }
$$

and

$$
\frac{V_{N}-\mathbb{E} V_{N}}{\sqrt{\operatorname{var}\left(V_{N}\right)}} \text { converges to a centered Gaussian variable }
$$

as $N \rightarrow \infty$.

Proof of Proposition 6.8. The generalized quadratic variation $V_{N}$ can be written

$$
\begin{aligned}
V_{N} & =\sum_{p=0}^{N-K}\left(\sum_{k=0}^{K} a_{k} X\left(\frac{k+p}{N}\right)\right)^{2} \\
& =\operatorname{Tr}\left({ }^{t} Y Y\right) .
\end{aligned}
$$

where $Y$ is the $\mathbb{R}^{N-K+1}$ valued random vector defined by $Y_{p, N}=\sum_{k=0}^{K} a_{k} X\left(\frac{k+p}{N}\right)$. Since $M=\mathbb{E}\left(Y^{t} Y\right)$ is a $(N-K+1) \times(N-K+1)$ symmetric matrix, we can find a diagonal matrix $\operatorname{Diag}\left(\lambda_{p, N}\right)$ with non negative eigenvalues $\lambda_{p, N}$ of $M$ on the diagonal, and an orthogonal $(N-K+1) \times(N-K+1)$ matrix $O$ such that

$$
\operatorname{Diag}\left(\lambda_{p, N}\right)={ }^{t} O M O \text {. }
$$

Let $\xi$ be a random vector defined by

$$
\xi=\operatorname{Diag}\left(\frac{1}{\sqrt{\lambda_{p, N}}}\right) O Y .
$$

Then the $\xi_{p}(p=0, \ldots, N-K)$ are identically independent centered Gaussian variables with variance 1, and

$$
\begin{aligned}
V_{N} & =\operatorname{Tr}\left({ }^{t} Y Y\right) \\
& =\sum_{p=0}^{N-K} \lambda_{p, N} \xi_{p}^{2} .
\end{aligned}
$$

Hence

$$
\operatorname{var}\left(V_{N}\right)=\operatorname{var}\left(\xi_{0}^{2}\right) \sum_{p=0}^{N-K} \lambda_{p, N}^{2}
$$


Then

$$
\begin{aligned}
\mathbb{E}\left(V_{N}-\mathbb{E} V_{N}\right)^{4} & =\mathbb{E}\left(\xi_{0}^{2}-1\right)^{4} \sum_{p=0}^{N-K} \lambda_{p, N}^{4}+\sum_{p, p^{\prime}=0}^{N-K} \mathbb{E}\left(\xi_{0}^{2}-1\right)^{2} \lambda_{p, N}^{2} \lambda_{p^{\prime}, N}^{2} \\
& \leq C\left(\sum_{p=0}^{N-K} \lambda_{p, N}^{2}\right)^{2} \\
& =C \operatorname{var}^{2} V_{N} .
\end{aligned}
$$

Using Proposition 6.7 and Borel-Cantelli's lemma, the almost sure convergence is proved.

Let us now prove the second part of Proposition 6.8, which describes the rate of convergence in (6.18). The following central limit theorem is used

Lemma 6.9. Consider the sequence of variable $S_{N}$ defined by

$$
S_{N}=\sum_{p=0}^{N-K} \lambda_{p, N}\left(\xi_{p}^{2}-1\right)
$$

where the $\xi_{p}$ are i.i.d. centered normalized Gaussian variables and the $\lambda_{p, N}$ are positive. If $\max _{p=0, \ldots, N-K} \lambda_{p, N}=$ $o\left(\sqrt{\operatorname{var}\left(S_{N}\right)}\right)$, then $S_{N} / \sqrt{\operatorname{var}\left(S_{N}\right)}$ converges in distribution to a centered normalized Gaussian variable.

Lemma 6.9 is easily proved using a Taylor expansion of the characteristic function of $S_{N}$.

Consequently we only have to prove that $\max _{p=0, \ldots, N-K} \lambda_{p, N}=o\left(\sqrt{\operatorname{var}\left(V_{N}\right)}\right)$. In order to bound the largest eigenvalue of the correlation matrix, a classical linear algebra lemma is used, that claims that the largest eigenvalue of a matrix $C$ is bounded by $\max _{i} \sum_{j}\left|C_{i, j}\right|$. Applying this Lemma to matrix $M=\left(m_{p, p^{\prime}}\right)$ leads to consider

$$
\begin{aligned}
m_{p, p^{\prime}} & =\mathbb{E}\left(Y_{p, N} Y_{p^{\prime}, N}\right) \\
& =\mathbb{E} \sum_{k, k^{\prime}=0}^{N-K} a_{k} a_{k^{\prime}} X\left(\frac{k+p}{N}\right) X\left(\frac{k^{\prime}+p^{\prime}}{N}\right) .
\end{aligned}
$$

By Lemma 6.5

$$
\begin{aligned}
\sum_{p^{\prime}=0}^{N-K}\left|\mathbb{E} \sum_{k, k^{\prime}=0}^{K} a_{k} a_{k^{\prime}} X\left(\frac{k+p}{N}\right) X\left(\frac{k^{\prime}+p^{\prime}}{N}\right)\right| & \leq \sum_{p^{\prime}=0}^{N-K} \frac{C}{N^{2 H}\left(1+\left(p-p^{\prime}\right)^{2}\right)} \\
& \leq \frac{C}{N^{2 H}} .
\end{aligned}
$$

Hence

$$
\max _{p=0, \ldots, N-K} \sum_{p^{\prime}=0}^{N-K}\left|\mathbb{E} \sum_{k, k^{\prime}=0}^{K} a_{k} a_{k^{\prime}} X\left(\frac{k+p}{N}\right) X\left(\frac{k^{\prime}+p^{\prime}}{N}\right)\right|=o\left(\sqrt{\operatorname{var}\left(V_{N}\right)}\right) .
$$

and Lemma 6.9 is applied to get the convergence in distribution of $\frac{V_{N}-\mathbb{E} V_{N}}{\sqrt{\operatorname{var}\left(V_{N}\right)}}$ in Proposition 6.8. With the same arguments, the asymptotic normality of a linear combination of $V_{N}$ and $V_{N / 2}$ with positive weights. The same can be of course done with negative weights. 
Denote by $P_{n}$ (resp. $\Psi_{n}$ ) the distribution (resp. the Laplace transform) of the couple $\left(V_{N}, V_{N / 2}\right)$. We know that $\Psi_{n}(x, y)$ converges to some $\Psi(x, y)$ when $x y \geq 0$, where $\Psi$ is the Laplace transform of a Gaussian distribution $\Phi$. Assume that $P_{n}$ converges to a distribution $P$. The Laplace transform of $P$ is equal to $\Psi(x, y)$ when $x y \geq 0$. The Laplace transform is defined on a convex set, so this set is the whole plan. It is analytic in its support, so it is equal to $\Psi$ on the whole plan. The unique possible limiting distribution is $\Psi$. But the sequence is tight, because its marginals are tight, so it converges to the Gaussian distribution $\Psi$.

The previous results are then applied to the estimator of $H$. Its convergence (a.s.) to $H$ is a consequence of the logarithmic behavior of $\mathbb{E} V_{N}$ and of (6.18). To estimate the rate of convergence of $\widehat{H}_{N}$ to $H$, we obtain a central limit theorem for $\sqrt{N}\left(\widehat{H}_{N}-\mathbb{E} \widehat{H}_{N}\right)$, which is a function of the couple $\left(V_{N}, V_{N / 2}\right)$. We deduce this asymptotic normality from the remark above and Theorem 3.311 of [34]. The proof is completed by showing that the bias term $\left|\mathbb{E} \widehat{H}_{N}-H\right|$ is of order $O\left(N^{-\eta}\right)$ which is preponderant since $\eta \geq 1 / 2$.

\subsubsection{Optimality of the sequence a}

The asymptotical variance of estimator (6.6) is known [58]. The best sequence $a_{k}, k=0, \ldots, K$, i.e. the sequence that minimizes this asymptotical variance, is unknown at the moment. [24,25] has computed this asymptotical variance for a lot of discrete sequences $a_{k}, k=0, \ldots, K$. The best results are obtained for the following sequences

(1) $0<H<3 / 4$.

$$
\begin{aligned}
& a_{0}=1, \\
& a_{1}=-1 .
\end{aligned}
$$

(2) $3 / 4 \leq H<1$.

$$
\begin{aligned}
& a_{0} \sim 0.4829629, \\
& a_{1} \sim-0.8365163, \\
& a_{2}=0.22414386, \\
& a_{3} \sim 0.12940952 .
\end{aligned}
$$

Notice that the two sequences are the discrete sequences associated with the Haar basis and the so-called Daubechies-4 wavelet basis [36].

\subsubsection{Filtered White Noises of arbitrary fractional index}

For the sake of simplicity, the Filtered White Noises have been introduced with a fractional index $0<H<1$. Indeed there is no reason for this restriction. We will now introduce Filtered White Noises of arbitrary fractional index $H>0$. Let

$$
e_{n}(\lambda)=\mathrm{e}^{\mathrm{i} \lambda}-\sum_{k=0}^{n} \frac{(i \lambda)^{k}}{k !}
$$

and

$$
g(t, \lambda)=\frac{a(t)}{|\lambda|^{\frac{1}{2}+H}}+\varepsilon(t, \lambda)
$$

Integer $n$ is chosen such that function $g(t, \lambda) e_{n}(\lambda)$ is square integrable for each $t$. The case $n=0$ correspond to the previous case $0<H<1$. Assumption 6.2 has to be generalized. 


\section{A.6.10.}

- $n+1>\beta>\alpha>n$ and $\varepsilon(t, \lambda) \in C^{2(n+1), 2}([0,1] \times \mathbb{R}) \quad$ is a function such that,

$$
\left|\frac{\partial^{i+j}}{\partial t^{i} \partial \lambda^{j}} \varepsilon(t, \lambda)\right| \leq \frac{C}{|\lambda|^{\frac{1}{2}+\eta+j}},
$$

for $i=0$ to $2(n+1)$ and $j=0$ to 2 with $\eta>1 / 2+\alpha$.

- $a \in C^{2 n+2}([0,1])$.

Now let $a_{k}, k=0, \ldots, K$ be a discrete sequence of real satisfying, with $J \geq 4 n+2$

$$
\begin{aligned}
\sum_{k=0}^{K} a_{k} & =0, \\
\sum_{k=0}^{K} k^{j} a_{k} & =0, \quad 1 \leq j \leq J, \\
\sum_{k=0}^{K} k^{J+1} a_{k} & \neq 0 .
\end{aligned}
$$

Generalized quadratic variations of process $X$ on $[0,1]$ at scale $1 / N$ associated with sequence $\left(a_{k}\right)$ are still defined by

$$
V_{N}=\sum_{p=0}^{N-K}\left(\Delta_{p} X\right)^{2},
$$

where

$$
\Delta_{p} X=\sum_{k=0}^{K} a_{k} X\left(\frac{k+p}{N}\right) .
$$

The estimator of parameter $H$ derived from the generalized quadratic variations is still as follows

$$
\widehat{H}_{N}=\frac{1}{2}+\frac{1}{2} \log _{2} \frac{V_{N / 2}}{V_{N}}
$$

Theorem 6.11. Let $X$ be a process satisfying A.6.10.

(1) Strong consistency.

$$
\lim _{N \rightarrow+\infty} \widehat{H}_{N} \stackrel{(\text { a.s. })}{=} H \text {. }
$$

(2) Asymptotic normality.

As $N \rightarrow+\infty, \sqrt{N}\left(\widehat{H}_{N}-H\right)$ converges in distribution to a centered Gaussian variable.

The proof of Theorem 6.11 is identical to the Proof of Theorem 6.3 and is therefore omitted. 


\section{Simulation}

\subsection{Cholevsky's method}

Most of the algorithms use the simulations of i.i.d. Gaussian r.v.'s. Let us therefore recall how to simulate Gaussian r.v.'s from uniform r.v.'s Let $X$ and $Y$ be two independent uniform variables on $[0,1]$. Then,

$$
(X, Y)=\left(\sqrt{-2 \log U_{1}} \cos \left(2 \pi U_{2}\right), \sqrt{-2 \log U_{1}} \sin \left(2 \pi U_{2}\right)\right)
$$

is a standard Gaussian centered vector.

Let $X$ be a centered Gaussian field with covariance function $R\left(M, M^{\prime}\right), M, M^{\prime} \in \mathcal{M}$. Let $M_{p}, p=0, \ldots, N$ be a discretization grid of $\mathcal{M}$. The vector $X\left(M_{p}\right), p=0, \ldots, N$ is a centered Gaussian vector with covariance matrix $\Sigma$

$$
\Sigma_{p, p^{\prime}}=R\left(M_{p}, M_{p^{\prime}}\right) \quad p, p^{\prime}=0, \ldots, N .
$$

The matrix $\Sigma$ can be decomposed into $\Sigma=L^{t} L$, where $L$ is a lower triangular matrix. Let $Z_{p}, p=0, \ldots, N$ be standard centered Gaussian r.v. and put $Z={ }^{t}\left(Z_{0}, \ldots, Z_{N}\right)$. We can then easily check that $L Z$ is a centered Gaussian vector with covariance matrix $\Sigma$. This method therefore allows to give an error-free simulation of the $X\left(M_{p}\right), p=0, \ldots, N$. This method has a complexity of order $O\left(N^{3}\right)$. Where $N$ is rather small, the Cholevski method can be used. Unfortunatly, when $N$ becomes large, this method is no more usable. Especially, this is the case when one wants to simulate a Gaussian field.

\subsection{Euclidean case: fieldsim method}

\subsubsection{Random midpoint displacement and refinements}

7.2.1.1. Random midpoint displacement

Brownian motion has independent, stationary and Gaussian increments. It is therefore straightforward to simulate a Brownian motion over a given discrete uniform grid. This simulation is based on standard results in Gaussian regression. Here is a brief summary. We will indicate here how to simulate a sample path of a Brownian motion at point $\frac{k}{2^{j}}$ by induction. We set $B(0)=0 . B(1)$ is chosen from a centered standard Gaussian variable. Next, $B(1 / 2)$ is selected from a Gaussian variable with mean $(B(0)+B(1)) / 2$ and variance $1 / 2$. At the $j$ th stage, the value $B\left(k / 2^{j}\right)$ (k odd) is simulated from an independant Gaussian variable with mean $\left(B\left((k-1) / 2^{j}\right)-B\left(k / 2^{j}\right)\right) / 2$ and variance $2^{-j}$.

The random midpoint displacement cannot be generalized to the fractional case: if we take $B\left(k / 2^{j}\right)$ from a Gaussian variable with mean $\left(B\left((k-1) / 2^{j}\right)-B\left(k / 2^{j}\right)\right) / 2$ and variance $2^{-j H}$, the resulting function fails to have stationary increments.

7.2.1.2. Refinements: the R-procedure fieldsim, available via [21]

Accurate simulation step. We first present the accurate simulation part of the procedure. Given a (regular) space discretization $\left\{M^{i}, i \in I\right\}$ of size $n_{I}$, the problem consists in giving a sample of a centered Gaussian vector of size $n_{I}:\left(X\left(M^{i}\right)\right)_{i \in I}$ of covariance matrix $\mathbf{R}$ given by $\mathbf{R}_{i, j}=R\left(M^{i}, M^{j}\right), i, j \in I$. We use the Cholevski method.

Refined simulation step. We need to introduce some additional notations. Let $X_{\mathcal{X}_{I}}(M)$, denote the orthogonal projection of $X(M)$ on the closed linear subspace $\mathcal{X}_{I}=\overline{s p}\left\{X\left(M^{i}\right), i \in I\right\}$, i.e. the linear predictor of $X(M)$ given $X\left(M^{i}\right), i \in I$. The partial innovation $X(M)-X_{\mathcal{X}_{I}}(M)$ is denoted by $\varepsilon_{\mathcal{X}_{I}}(M)$. Since $\varepsilon_{\mathcal{X}_{I}}(M)$ is uncorrelated with any variables of the space $\mathcal{X}_{I}$, we can obtain "accurate" simulation of $X(M)$ by $X_{\mathcal{X}_{I}}(M)+\sqrt{\operatorname{Var}\left(\varepsilon_{\mathcal{X}_{I}}(M)\right)} U$ where $U$ is a centered and reduced Gaussian variable independent of $X\left(M^{i}\right), i \in I$. Notice that the coefficients weights, the variables $X\left(M^{i}\right), i \in I$ in $X_{\mathcal{X}_{I}}(M)$ and the variance of the partial innovation may be determined from the second order structure of the sequence $X\left(M^{i}\right), i \in I, X(M)$ (see [37] for details). The drawback of this 
midpoint $\mathrm{H}=0.1$

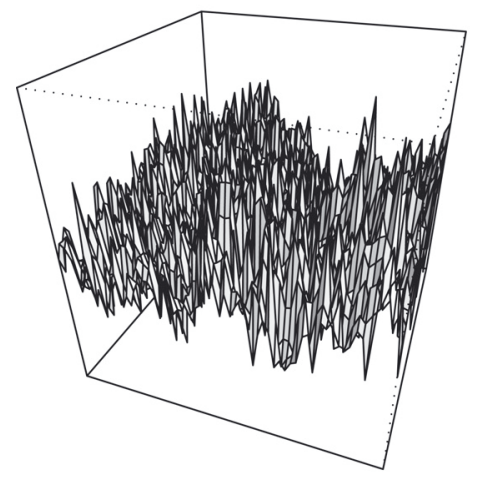

midpoint $\mathrm{H}=0.5$

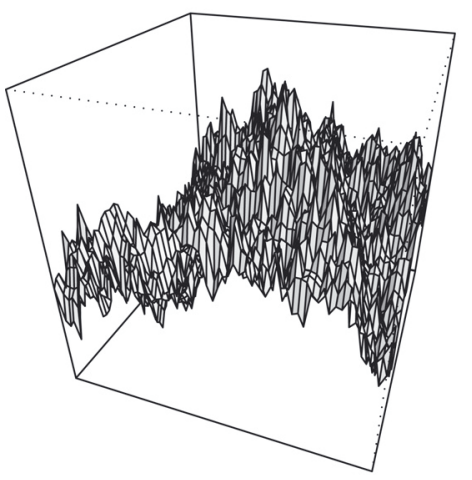

midpoint $\mathrm{H}=0.9$

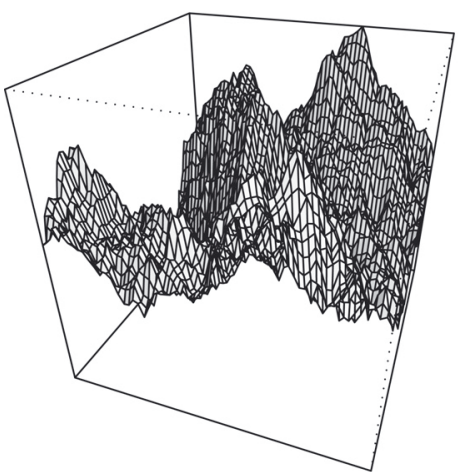

fieldsim $\mathrm{H}=0.1$

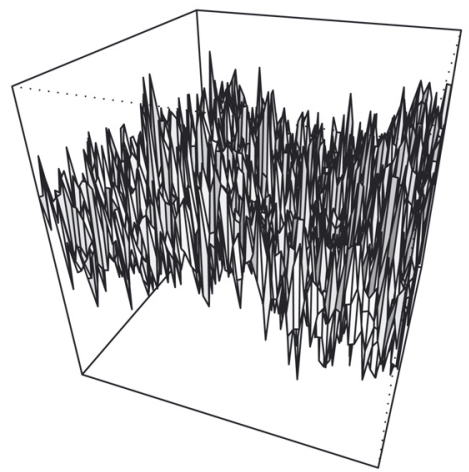

fieldsim $\mathrm{H}=0.5$

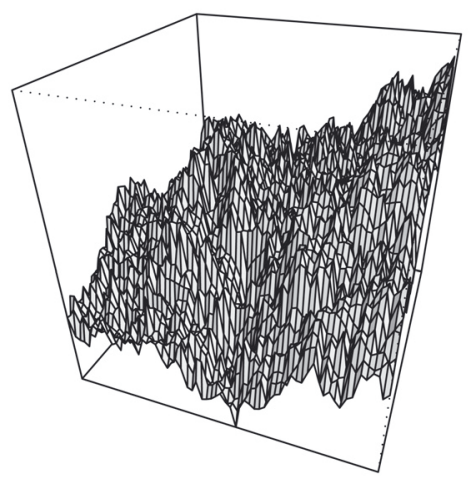

fieldsim $\mathrm{H}=0.9$

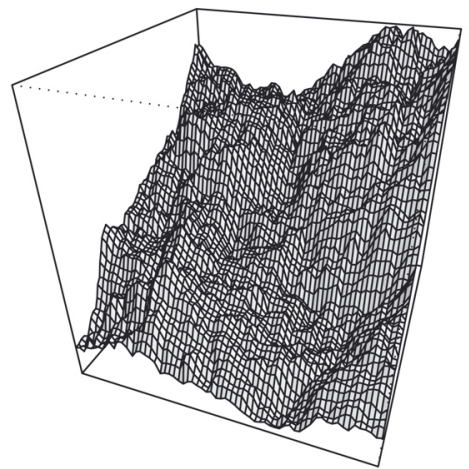

Figure 1. Fractional Brownian fields with indexes 0.1, 0.5, 0.9.

approach is when the simulated sequence size increases, we have to stock more and more quantities (filters of several partial innovation and associated variances) and to do more and more calculus. Even if that can be done in the $d=1$ case, it becomes numerically unfeasible when $d \geq 2$. A natural approach to overcome this problem, is to replace in the previous procedure the indexes set $I$ by a set of indexes of neighbors of $M$. We denote by $N_{M}$ this set. Notice that $X_{\mathcal{X}_{N_{M}}}(M)$ is the best linear combination of variables of $\mathcal{X}_{N_{M}}$ approximating $X(M)$ 


\section{Multifractional Brownian Field $\mathrm{H}(\mathrm{t})=0.5$}
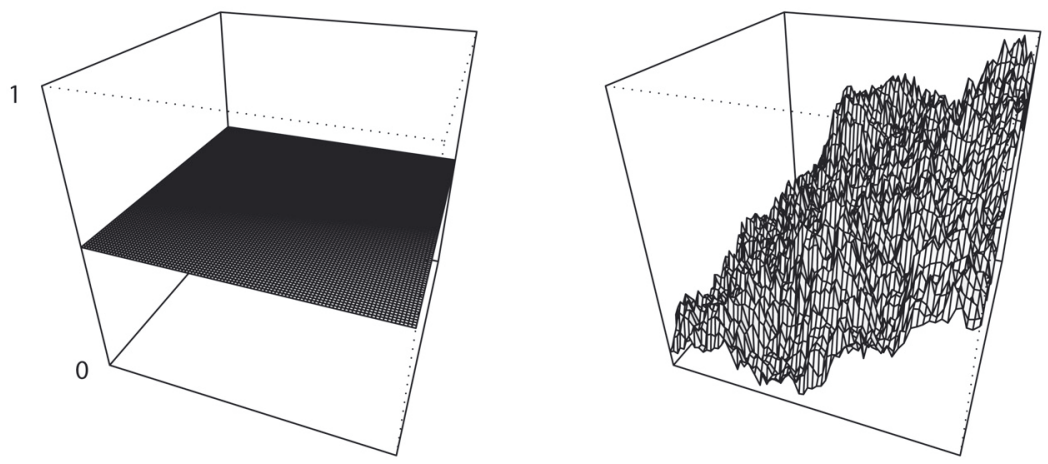

Multifractional Brownian Field $H(t)=0.5+0.4 t$
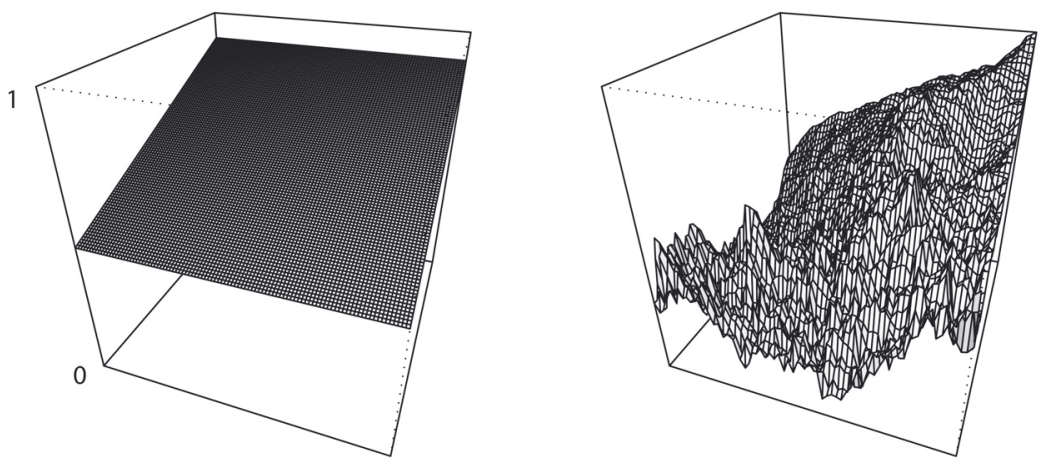

Multifractional Brownian Field $H(t)=0.7+0.2^{*} \sin (6 . \pi . t)$
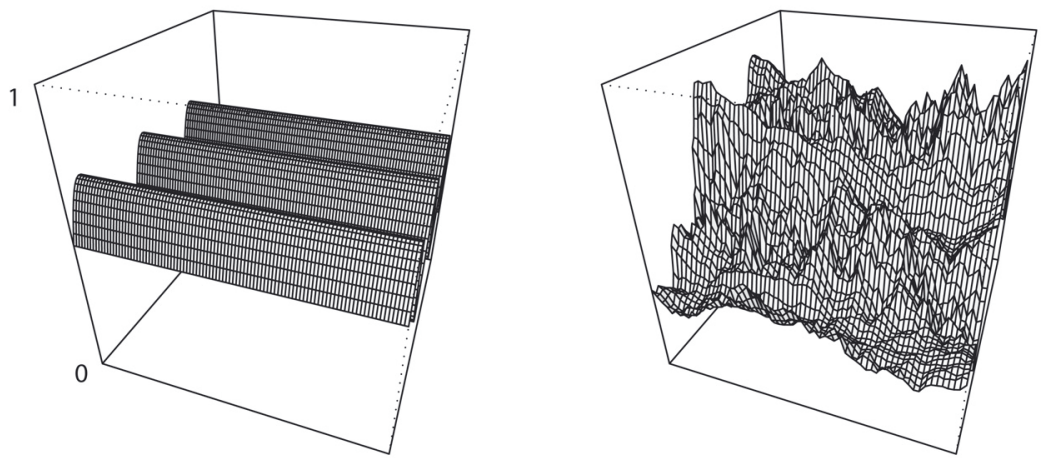

Figure 2. Multifractional Brownian fields, $c f$. $[8,69]$ (on the right) for functions $H(t)=0.5$, $H(t)=0.5+0.4 t_{1}$ and $H(t)=0.7+0.2 \sin \left(2 \pi t_{1}\right)$ (on the left).

in the sense that the variance of $X(M)-X_{\mathcal{X}_{N_{M}}}(M)$ is minimum. If we have to use only some variables of the set $\mathcal{X}_{N_{M}}$ in order to obtain simulation of $X(M)$, the best way is to use $X_{\mathcal{X}_{N_{M}}}(M)+\sqrt{\operatorname{Var}\left(\varepsilon_{\mathcal{X}_{N_{M}}}(M)\right)} U$. Let us remark that such a simulated process does not admit anymore $R(\cdot, \cdot)$ as a covariance function, but a covariance function that is a good approximation of $R(\cdot, \cdot)$. 


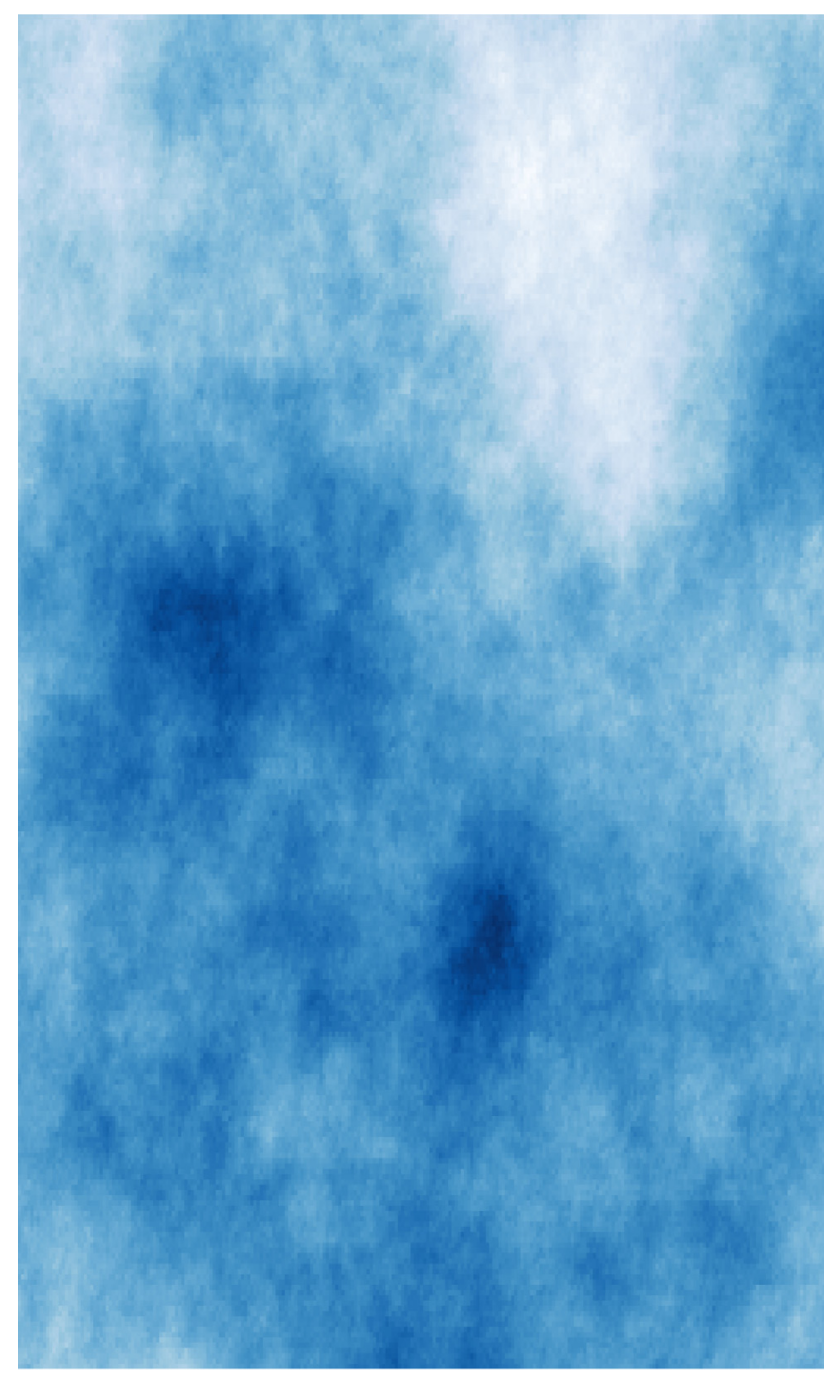

Figure 3. Cloud simulation: fractional Brownian fields with index 0.5.

\subsubsection{Examples}

Two representations have been used: the fields can be represented by a random surface, or by a random coloured texture. Especially, a blue to white choice leads to cloud simulator.

\subsection{The procedure fieldsim adapted to manifolds}

The previous procedure can be adapted to the case of fields indexed by a manifold, see [22]. The main problem stands in the discretization grid choice. There is, in the case of the sphere for instance, no equidistributed grid and it is difficult to define a concept of finer grid such as in the case of field indexed by $[0,1]^{2}$. Moreover, the choice of a discretization grid can be related to the software that one wishes to use to represent the manifold.

We develop a procedure for which the user enters a grid of his/her choice for each step (accurate and refined) and a distance to be used in order to determine the neighbors. Thus one can use our procedure for any manifold 


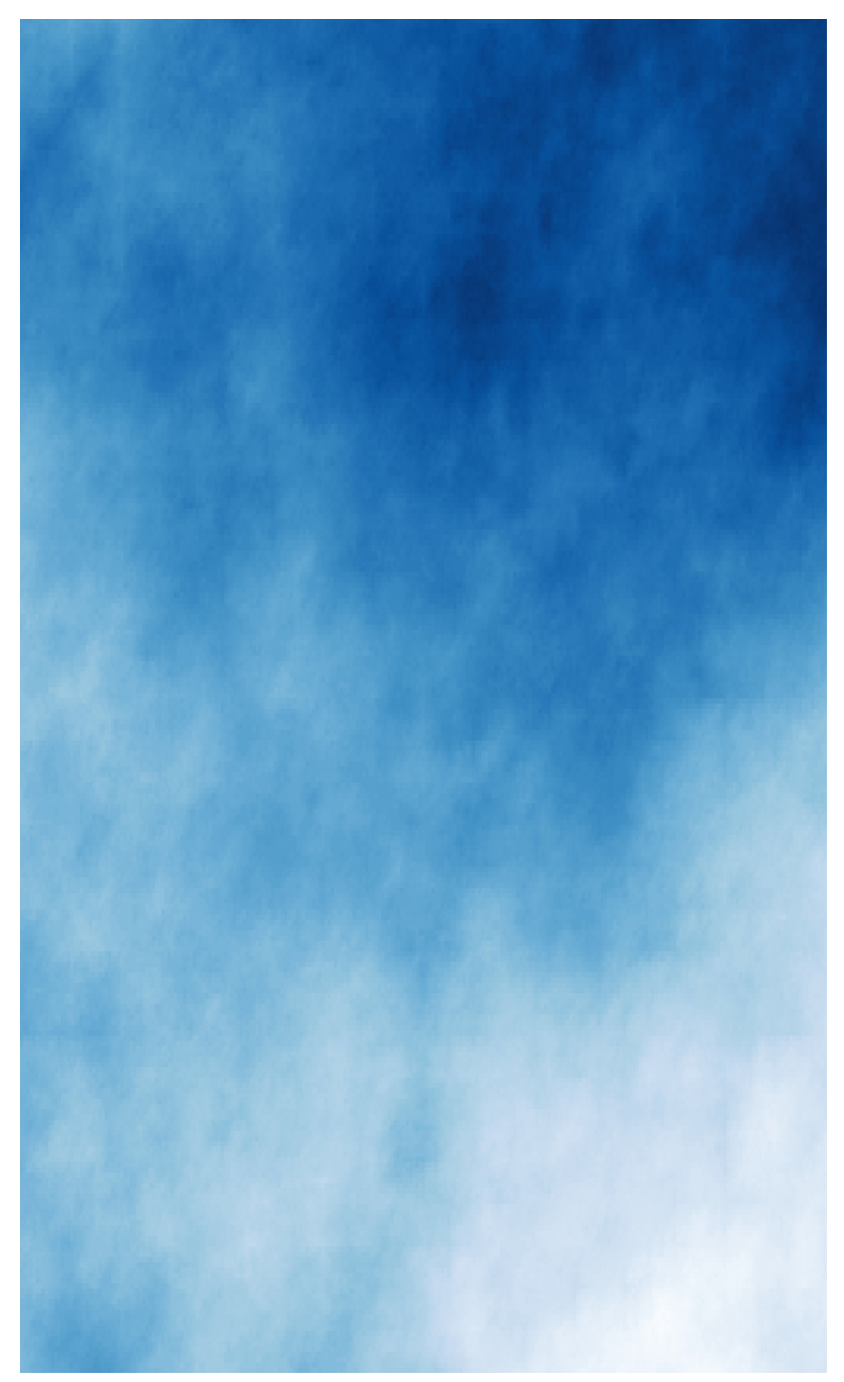

Figure 4. Cloud simulation: fractional Brownian fields with index 0.7.

$\mathcal{M}$ once a covariance function, a grid and a distance are given. We give here two examples of manifolds: the sphere and the hyperboloïd.

Let us precise how we use it for these two manifolds. We refer to [22] for detailed results. We denote by $\mathcal{S}_{g}$ the set of the point of $\mathcal{M}$ at which we want to generate the process (the visualization grid). This set choice can be induced for instance by the software that one wishes to use to represent the manifold. Let us recall that we want to generate a sample of field indexed by the manifold $\mathcal{M}$ (discretized at $\mathcal{S}_{g}$ ) and with covariance function $R(\cdot, \cdot)$. We need also to specify the concept of neighbors. A natural choice is to use the geodesic distance between two points. So the closest neighbors of some point $M$ are the points closest to $M$ according to this distance. In general, the cardinal of $\mathcal{S}_{g}$ is too large to use only the accurate simulation step. Moreover in contrast to the grid chosen in the case of field indexed by $[0,1]^{2}$, one is not able in general to use any more a concept of finer grid. Indeed, for our sphere visualization grid for instance, using overlapping sub-grids (an atlas of 6 maps), each sub-grid have some parts of the sphere with very few points and there are some others with points accumulation (in particular on the six poles). That is why in this case, we propose to first simulate the process at uniform 

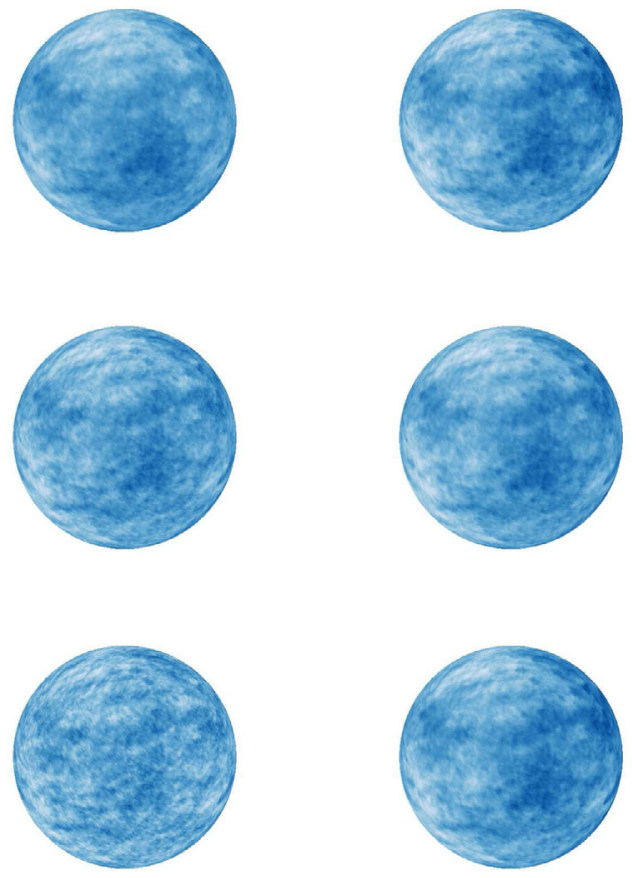

FiguRE 5. On the left, spheres indexed fractional fields with covariance function $R_{1}$ and Hölder index $H=0.45$ (top), $H=0.3$ (middle) and $H=0.15$ (bottom). On the right, sphere indexed fractional fields of Hölder index $H=0.45$ with covariance function $R_{2}$ (top), $R_{3}$ (middle) and $R_{4}$ (bottom). All simulations are done with $N_{e}=100, N_{r}=1000, N_{g}=100$ and nbNeighbor $=15$.
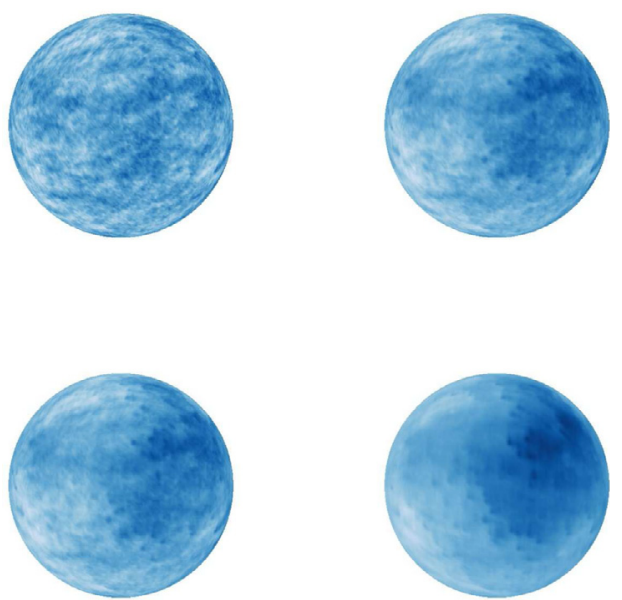

FiguRE 6. Sphere indexed fractional fields with covariance function $R_{5}$ and Hölder index $H=0.2$ (top left), $H=0.45$ (top right), $H=0.5$ (bottom left) and $H=0.8$ (bottom right). All simulations are done with $N_{e}=100, N_{r}=1000, N_{g}=100$ and $n b N$ eighbor $=15$. 

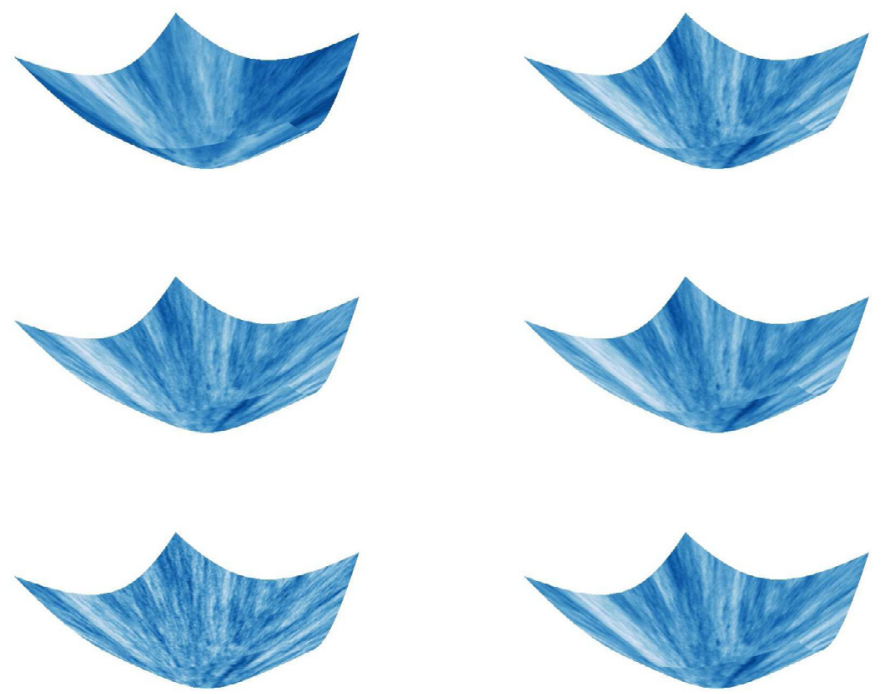

Figure 7. On the left, hyperboloid indexed fractional fields with covariance function $R_{1}$ and index $H=0.45$ (top), $H=0.3$ (middle) and $H=0.15$ (bottom). On the right, hyperboloid indexed fractional fields of index $H=0.45$ with covariance function $R_{2}$ (top), $R_{3}$ (middle) and $R_{4}$ (bottom). All simulations are done with $N_{e}=100, N_{r}=1000, N_{g}=150$ and $n b N$ eighbor $=15$.

random points and next to simulate the process at the $\mathcal{S}_{g}$ points. Let $\mathcal{S}_{u}$ the uniform random points set. We propose to run the procedure fieldsim with the set $\left\{\mathcal{S}_{u}, \mathcal{S}_{g}\right\}$. This set $\left\{\mathcal{S}_{u}, \mathcal{S}_{g}\right\}$ is cut out into the set $\left\{\mathcal{S}_{e}, \mathcal{S}_{r}\right\}$. $N_{e}, N_{r}, N_{g}$ are the cardinal of $\mathcal{S}_{e}, \mathcal{S}_{r}, \mathcal{S}_{g} .(X(M))_{M \in \mathcal{S}_{g}}$ is simulated using nbNeighbor neighbors.

In the next figures, field with the following convariance function has been simulated, both in spherical and hyperbolic case.

$$
\begin{aligned}
& R_{1}\left(M, M^{\prime}\right)=1 / 2\left\{d^{2 H}(O, M)+d^{2 H}\left(O, M^{\prime}\right)-d^{2 H}\left(M, M^{\prime}\right)\right\} \\
& R_{2}\left(M, M^{\prime}\right)=\exp \left(-d^{2 H}\left(M, M^{\prime}\right)\right), \\
& R_{3}\left(M, M^{\prime}\right)=\ln \left(1+d^{2 H}(O, M)\right)+\ln \left(1+d^{2 H}\left(O, M^{\prime}\right)\right)-\ln \left(1+d^{2 H}\left(M, M^{\prime}\right)\right), \\
& R_{4}\left(M, M^{\prime}\right)=\frac{1}{1+d^{2 H}\left(M, M^{\prime}\right)} .
\end{aligned}
$$

Finally we can restrict the Euclidean fractional Brownian fields indexed by the sphere. We obtain the following covariance function:

$$
R_{5}\left(M, M^{\prime}\right)=1-2^{2 H-1}\left(\sin \left(\frac{d\left(M, M^{\prime}\right)}{2}\right)\right)^{2 H}
$$

where $H \in(0,1)$.

Acknowledgements. I am grateful to an anonymous referee for valuable comments. 


\section{REFERENCES}

[1] P. Abry, P. Gonçalvès and P. Flandrin, Wavelets, spectrum analysis and 1/f processes. Lect. Note Stat. 103 (1995) 15-29.

[2] A. Ayache and J. Lévy-Vehel, The Multifractional Brownian motion. Stat. Inference Stoch. Process. 1 (2000) 7-18.

[3] A. Ayache and J. Lévy-Vehel, On the identification of the pointwise Hölder exponent of the generalized multifractional Brownian motion. Stoc. Proc. Appl. 111 (2004) 119-156.

[4] A. Ayache, P. Bertrand and J. Lévy-Vehel, A central limit theorem for the generalized quadratic variation of the step fractional Brownian. Stat. Inference Stoch. Process. 10 (2007) 1-27.

[5] J.-M. Bardet, Testing for the presence of self-similarity of Gaussian time series having stationary increments. J. Time Ser. Anal. 25 (2000) 497-515.

[6] J.-M. Bardet and P. Bertrand, Identification of the multiscale fractional Brownian motion with biomechanical applications. J. Time Ser. Anal. 28 (2007) 1-52.

[7] B. Bekka, P. de la Harpe and A. Valette, Kazhdan's Property (T). Cambridge University Press (2008).

[8] A. Benassi, S. Jaffard and D. Roux, Gaussian processes and Pseudodifferential Elliptic operators. Revista Mathematica Iberoam. 13 (1997) 19-90.

[9] A. Benassi, S. Cohen and J. Istas, Identifying the multifractional function of a Gaussian process. Stat. Probab. Lett. 39 (1998) 337-345.

[10] A. Benassi, S. Cohen, J. Istas and S. Jaffard, Identification of filtered white noises. Stoc. Proc. Appl. 75 (1998) 31-49.

[11] A. Benassi, P. Bertrand, S. Cohen and J. Istas, Identification of the Hurst index of a step fractional Brownian motion. Stat. Inference Stoch. Process 3 (2000) 101-111.

[12] A. Benassi, S. Cohen and J. Istas, Identification and properties of real harmonizable fractional Lévy motions. Bernoulli 8 (2002) 97-115.

[13] A. Benassi, S. Cohen and J. Istas, On roughness indices for fractional fields. Bernoulli 10 (2004) 357-373.

[14] A. Begyn, Quadratic variations along irregular subdivisions for Gaussian processes. Electron. J. Probab. 10 (2005) 691-717.

[15] A. Begyn, Asymptotic expansion and central limit theorem for quadratic variations of Gaussian processes. Bernoulli 13 (2007) $712-753$.

[16] A. Begyn, Functional limit theorems for generalized quadratic variations of Gaussian processes. Stoc. Proc. Appl. 117 (2007) 1848-1869.

[17] C. Berzin and J. Leon, Estimating the Hurst parameter. Stat. Inference Stock. Process. 10 (2007) 49-73.

[18] A. Bonami and A. Estrade, Anisotropic analysis of Gaussian models. J. Fourier Anal. Appl. 9 (2004) $215-236$.

[19] V. Borrelli, F. Cazals and J.-M. Morvan, On the angular defect of triangulations and the pointwise approximation of curvatures, curves and surfaces'02. Comput. Aid. Geom. Des. 20 319-341.

[20] J. Bretagnolle, D. Dacunha-Castelle and J.-L. Krivine, Lois stables et espaces $L^{p}$. Ann. Inst. Henri Poincaré 2 (1969) $231-259$.

[21] A. Brouste, J. Istas and S. Lambert-Lacroix, On fractional Gaussian random fields simulation. J. Stat. Soft. 1 (2007) 1-23.

[22] A. Brouste, J. Istas and S. Lambert-Lacroix, On simulation of fractional Brownian motion indexed by a manifold. J. Stat. Soft. 36 (2010).

[23] N. Chentsov, Lévy's Brownian motion of several parameters and generalized white noise. Theory Probab. Appl. 2 (1957) 265-266.

[24] J.-F. Coeurjolly, Simulation and identification of the fractional Brownian motion: a bibliographical and comparative study. $J$. Stat. Software 5 (2000) 1-53.

[25] J.-F. Coeurjolly, Estimating the parameters of a fractional Brownian Motion by discrete variations of its sample paths. Stat. Inference Stoch. Process. 4 (2001) 199-227.

[26] J.-F. Coeurjolly, Identification of multifractional Brownian motion. Bernoulli 11 (2005) 987-1008.

[27] J.-F. Coeurjolly, Hurst exponent estimation of locally self-similar Gaussian processes using sample quantiles. Ann. Statist. 36 (2008) 1404-1434.

[28] J.-F. Coeurjolly and J. Istas, Cramer-Rao bounds for fractional Brownian motions. Stat. Probab. Lett. 53 (2001) $435-447$.

[29] S. Cohen, From self-similarity to local self-similarity: the estimation problem. Fractal in Engineering, edited by J. Lévy-Vehel and C. Tricot. Springer Verlag, Delft (1999).

[30] S. Cohen and J. Istas, An universal estimator of local self-similarity. Preprint (2006).

[31] S. Cohen and J. Istas, Fractional fields: Modelling and statistical applications (Submitted).

[32] S. Cohen and M. Lifshits, Stationary Gaussian random fields on hyperbolic spaces and Euclidean spheres. To appear in ESAIM: $P S$.

[33] S. Cohen, X. Guyon, O. Perrin and M. Pontier, Singularity functions for fractional processes: application to the fractional brownian sheet. Ann. Inst. Henri Poincaré 42 (2006) 187-205.

[34] D. Dacunha-Castelle and M. Duflo, Probabilités et Statistiques tome 2. Masson, Paris (1983).

[35] R. Dalhaus, Efficient parameter estimation for self-similar processes. Ann. Statist. 17 (1989) 1749-1766.

[36] I. Daubechies, Orthonormal bases of compactly supported wavelets. Commun. Pure Appl. Math. 41 (1988) $909-996$.

[37] S. Dégerine and S. Lambert-Lacroix, Partial autocorrelation function of a nonstationary time series. J. Multiv. Anal. (2003) $46-59$. 
[38] R.L. Dobrushin, Automodel generalized random fields and their renorm group, in Multicomponent Random Systems, edited by R.L. Dobrushin and Ya. G. Sinai. Dekker, New York (1980) 153-198.

[39] A. Dress, V. Moulton and W. Terhalle, T-theory: An overview, Eur. J. Comb. 17 (1996) 161-175.

[40] A. Erdélyi, W. Magnus, F. Oberhettinger and F. Tricomi, Higher transcendental functions (Bateman manuscript project). McGraw-Hill 2 (1953)

[41] K. Falconer, Tangent fields and the local structure of random fields. J. Theor. Probab. 15 (2002) 731-750.

[42] K. Falconer, The local structure of random processes. J. Lond. Math. Soc. 67 (2003) 657-672.

[43] J. Faraut, Fonction brownienne sur une variété riemannienne. Séminaire de probabilités de Strasbourg 7 (1973) 61-76.

[44] J. Faraut and H. Harzallah, Distances hilbertiennes invariantes sur un espace homogène. Ann. Inst. Fourier 24 (1974) $171-217$.

[45] S. Gallot, D. Hulin and J. Lafontaine, Riemannian Geometry, 2nd edition. Springer-Verlag (1993).

[46] R. Gangolli, Positive definite kernels on homogeneous spaces and certain stochastic processes related to Lévy's Brownian motion of several parameters. Ann. Inst. Henri Poincaré 3 (1967) 121-226.

[47] X. Guyon and J. Leon, Convergence en loi des H-variations d'un processus gaussien stationnaire. Ann. Inst. Henri Poincaré 25 (1989) 265-282.

[48] S. Helgason, Differential Geometry and Symmetric spaces. Academic Press (1962).

[49] E. Herbin and E. Merzbach, A set-indexed fractional Brownian motion. J. Theor. Probab. 19 (2006) $337-364$.

[50] E. Herbin and E. Merzbach, Stationarity and self-similarity characterization of the set-indexed fractional Brownian motion. J. Theor. Probab. 22 (2009) 1010-1029.

[51] J. Istas, Spherical and hyperbolic fractional Brownian motion. Electron. Comm. Probab. 10 (2005) 254-262.

[52] J. Istas, On fractional stable fields indexed by metric spaces. Electron. Comm. Probab. 11 (2006) $242-251$.

[53] J. Istas, Karhunen-Loève expansion of spherical fractional Brownian motions. Stat. Probab. Lett. 76 (2006) $1578-1583$.

[54] J. Istas, Quadratic variations of spherical fractional Brownian motions, Stoc. Proc. Appl. 117 (2007) 476-486.

[55] J. Istas, Identifying the anisotropical function of a d-dimensional Gaussian self-similar process with stationary increments. Stat. Inf. Stoc. Proc. 10-1 (2007) 97-106.

[56] J. Istas and C. Lacaux, On locally self-similar fractional random fields indexed by a manifold. preprint.

[57] J. Istas and G. Lang, Variations quadratiques et estimation de l'exposant de Hölder local d'un processus gaussien. C. R. Acad. Sci. Sér. I Paris 319 (1994) 201-206.

[58] J. Istas and G. Lang, Quadratic variations and estimation of the Hölder index of a Gaussian process. Ann. Inst. Henri Poincaré 33 (1997) 407-436.

[59] J. Kent and A. Wood, Estimating the fractal dimension of a locally self-similar Gaussian process using increments. J. Roy. Statist. Soc. B 59 (1997) 679-700.

[60] A. Koldobsky, Schoenberg's problem on positive definite functions. Algebra Anal. 3 (1991) 78-85.

[61] A. Koldobsky and Y. Lonke, A short proof of Schoenberg's conjecture on positive definite functions. Bull. Lond. Math. Soc. (1999) 693-699.

[62] A. Kolmogorov, Wienersche Spiralen und einige andere interessante Kurven im Hilbertsche Raum (German). C. R. (Dokl.) Acad. Sci. URSS 26 (1940) 115-118.

[63] C. Lacaux, Real harmonizable multifractional Lévy motions. Ann. Inst. Henri Poincaré 40 (2004) $259-277$.

[64] G. Lang and F. Roueff, Semi-parametric estimation of the Hölder exponent of a stationary Gaussian process with minimax rates. Stat. Inf. Stoc. Proc. 4-3 (2001) 283-306.

[65] P. Lévy, Processus stochastiques et mouvement Brownien. Gauthier-Vilars (1965).

[66] T. Lindstrom, Fractional Brownian fields as integrals of white noise. Bull. Lond. Math. Soc. 25 (1993) 83-88.

[67] M. Maejima, A remark on self-similar processes with stationary increments. Can. J. Stat. 14 (1986) 81-82.

[68] B.B. Mandelbrot and J.W. Van Ness, Fractional Brownian motions, fractional noises and applications, SIAM Rev. 10 (1968) 422-437.

[69] R. Peltier and J. Lévy-Vehel, Multifractional Brownian motion: definition and preliminary results. Rapport de recherche de l'INRIA 2645 (1996).

[70] P. Petersen, Riemannian Geometry. Graduate Texts in Mathematics, Springer (1998).

[71] E. Rafajlowicz, Testing (non-)existence of input-output relationships by estimating fractal dimensions. IEEE Trans. Signal Process. 52 (2004) 3151-3159.

[72] G. Robertson, Crofton formulae and geodesic distance in hyperbolic spaces. J. Lie Theory 8 (1998) $163-172$.

[73] G. Robertson and T. Steger, Negative definite kernels and a dynamical characterization of property $T$ for countable groups. Ergod. Theory Dyn. Syst. 18 (1998) 247-253.

[74] W. Rudin, Fourier analysis on groups. Wiley (1962).

[75] G. Samorodnitsky, Long memory and self-similar processes. Annales de la Faculté des Sciences Toulouse 15 (2006) $107-123$.

[76] G. Samorodnitsky and M. Taqqu, Stable non-Gaussian random processes: stochastic models with infinite variance. Chapman \& Hall, New York (1994).

[77] I. Schönberg, Metric spaces and positive definite functions. Ann. Math. 39 (1938) 811-841.

[78] R. Seeley, Spherical harmonics. Am. Math. Mon. 73 (1966) 115-121.

[79] S. Stoev and M. Taqqu, Stochastic properties of the linear multifractional stable motion. Adv. Appl. Prob. 36 (2004) $1085-1115$. 
[80] G. Szego, Orthogonal Polynomials, 4th edition, in Amer. Math. Soc. Providence, RI (1975).

[81] S. Takenaka, Integral-geometric construction of self-similar stable processes. Nagoya Math. J. 123 (1991) 1-12.

[82] S. Takenaka, I. Kubo and H. Urakawa, Brownian motion parametrized with metric space of constant curvature. Nagoya Math. J. 82 (1981) 131-140.

[83] A. Valette, Les représentations uniformément bornées associées à un arbre réel. Bull. Soc. Math. Belgique 42 (1990) 747-760.

[84] H. Wang, Two-point homogeneous spaces. Ann. Math. 2 (1952) 177-191.

[85] A. Yaglom, Some classes of random fields in $n$-dimensional space, related to stationary random processes. Theory Probab. Appl. 2 (1957) 273-320.

[86] A. Zaanen, Linear Anal. North Holland Publishing Co (1960). 